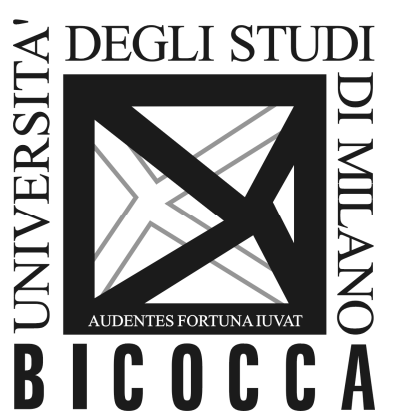

\author{
DEPARTMENT OF ECONOMICS, \\ MANAGEMENT AND STATISTICS \\ UNIVERSITY OF MILAN - BICOCCA
}

DEMS WORKING PAPER SERIES

\title{
A New Approach to Contest Models
}

Fausto Cavalli, Mario Gilli, Ahmad Naimzada

No. 364 - March 2017

Dipartimento di Economia, Metodi Quantitativi e Strategie di Impresa Università degli Studi di Milano - Bicocca 


\title{
A New Approach to Contest Models*
}

\author{
Fausto Cavalli ${ }^{\dagger}$ Mario Gilli ${ }^{\ddagger}$ and Ahmad Naimzada ${ }^{\ddagger}$
}

January 2017

\begin{abstract}
The purpose of this paper is to propose a symmetric two player general contest model in order to study the relationship between equilibria and crucial structural parameters of the model. In particular, given a general specification of the players' set of possible entries, of the agents' utility functions, and of the rules that presides over outcomes, we aim to analyze the characteristics of the set of equilibria as a function of structural characteristics of the contest technology and of the outcome function. Focusing on three main cases, we study the effect of introducing spillover in the marginal productivity of agents' efforts and in the polarization between agents' goals. Firstly, we show that without spillover the equilibrium efforts' intensity is uniquely connected to the ratio between marginal productivity of effort and polarization. Secondly, we are able to connect existence of multiple symmetric and asymmetric equilibria to the intensity of spillover effects into outcomes. Finally, we show that spillover in contest technology can imply the non existence of equilibria.
\end{abstract}

Keywords: symmetric contest, multiple equilibria, symmetric and asymmetric equilibria.

JEL Classification: C72 - D72 - D74

*We would especially like to thank Marco Serena for many very helpful comments and suggestions. We also thank the seminar participants at UECE 2014, Freiburg 2015 and EPCS 2016.

${ }^{\dagger}$ fausto.cavalli@unicatt.it, Department of Mathematical Sciences, Mathematical Finance and Econometrics, Catholic University of Milan, Via Necchi 9, 20123 Milan - Italy

${ }_{\ddagger}^{\ddagger}$ mario.gilli@unimib.it, ahmad.naimzada@unimib.it, Department of Economics, Management and Statistics, University of Milan-Bicocca, Piazza Dell'Ateneo Nuovo 1, 20126 Milan - Italy 


\section{Introduction}

Many interesting economic, social or political situations can be analyzed as games where players expend costly, non refundable resources trying to affect the probability of getting a desired result. These games are called contests and they model any situation where agents submit entries that influence the probability of reaching a desired outcome which bears some costs for the action; entries might be bids, efforts, commitments of non refundable resources. Contests are games with strong mutual externalities: a contestant expands efforts to increase the likelihood of getting the desired outcome, but at the same time this implies a reduced probability of getting the desired outcome by the other contestants. Other type of externalities might indeed emerge, e.g. when agents can directly sabotage or when the desired outcomes are directly affected by bids, however the externality effect on probabilities is the fundamental force at work in contests. The prevalence of contests in economic, social and political life is immediate: costly marketing and advertising competitions to get a bigger share of the market, tournaments or more general reward/punishment mechanisms in organizations, entertaining competitions (sports, talent shows etc.), rent-seeking situations, military, social and political conflicts, all-pay auctions, litigations, predation activities, political competition, R \& D competition, are all possible examples of application of contest theory. These seemingly different situations share a common content: they are different ways of conflicting to increase the likelihood to reach a desired outcome. In this general sense, conflicts are ubiquitous in social and political environments and are a significant part of human history, whether they take the form of wars, disputes, strikes, litigations, lobbying, competitions, tournaments, demonstrations, insurrections, terrorism. In this paper we will consider conflicts in this general sense as the paradigm of a contest.

Contests can be distinguished referring to several characteristics concerning their structure and the agents' possible choices. For instance, we can classify them depending on whether agents' choices are sequential or simultaneous, entry is endogenous or fixed, cost are exogenous or endogenous, and, more generally, specifying the set of possible choices, the utilities and the rules governing outcomes. The literature on contests is vast and we make no attempt to cover it in details, the interest reader should consult Corchon 2007, Konrad 2006 and Nitzan 1994. After the seminal contributions by Tullock 1967 and 1980 and Krueger 1974, a general analysis of rent-seeking games has been provided by Pérez-Castrillo 1992 and by Riaz et al. 1995, while Corchon 2007 and Konrad 2009 provide formal models for contests and review the main possible applications and generalizations.

We think the standard contest models suffer from two shortcomings. First, standard models have a unique interior fully stable equilibrium, as shown in Szidarovszky and Okuguchi 1997. This is unsatisfactory because the above phenomena modelled as contests, in reality often present characteristics that are not compatible with a unique interior equilibrium. For example, we might have contests with no bids or, vice versa, with maximum efforts. Again, we might have symmetric contests with asymmetric efforts. And also multiple symmetric equilibria might be interesting. We believe it is important to understand whether we might explain this heterogeneity in behavior as equilibrium phenomena. Second, even when we have multiple symmetric or asymmetric equilibria as in Chowdhury and Sheremeta 2011a and 2011b or in Cornes and Hartley 2012, the connection between the structural characteristics of the contest and the properties of the players' equilibrium behavior is obscure. And, again, zero effort equilibria are ruled out by assuming the Tullock contest success technology.

In this paper we present a unified framework to analyze the set of pure strategy Nash equilibria in two players, simultaneous move, symmetric general contest model where we specify several essential elements characterizing a contest, such as the players' set of possible entries, the agents' utility functions, and the rules that presides over outcomes. In particular, the crucial differences of the present model with respect to 
the previous modelling approaches concern the contest technology and the players' utility functions. Then, on the basis of such assumptions we examine some particular examples in order to investigate the properties of the set of equilibria, studying the relationship between equilibria and crucial structural parameters. In particular, we focus on the marginal productivity of agents' efforts (which concerns the contest technology), and the polarization between agents' goals, (which concerns the players' utility), with and without spillover. The most innovative part of our model is actually the combination of contest technology and of players' utility functions to get a simple but effective model that allows investigating the resulting equilibria configuration for general contests. We focus on three main examples, which exhibit interesting equilibria configurations and comparative statics and that make the role of some crucial contest parameters explicit. First, we show that, without spillover, the equilibrium efforts' intensity is uniquely connected to the ratio between marginal productivity of effort and polarization, and it can be zero, intermediate or maximum. Second, we are able to connect the existence of multiple symmetric and asymmetric equilibria to the intensity of spillover effects into outcomes. Finally, we show that spillover in contest technology might imply non existence of an equilibrium. Through these examples, we are able to better understand under what conditions we have unique or multiple or no equilibria, and the properties of these equilibria in terms of structural parameters.

The works that bear most resemblance with our contribution are the papers by Chowdhury and Sheremeta 2011a and 2011b, where the authors construct a generalized Tullock contest to analyze under what conditions their generalized contest has either a unique symmetric or multiple asymmetric equilibria. However, even if the research aims and the spillover effects are similar, the models and the results are quite different. Actually, we not only derive conditions such that we get multiple symmetric (and asymmetric) equilibria, but our modelling approach also allows connecting such equilibrium properties to structural parameters that have an immediate relation with the underlying characteristics of the contest.

From a methodological point of view, the properties of contests can be analyzed in two different and complementary ways: either using comparative statics showing how equilibria change as parameters change, or with out-of equilibrium dynamics to emphasize the complex and cyclic players' behavior. This paper belongs to a research strand in which we are pursuing both aspects. In this paper we analyze the structural conditions that generate one, zero or multiple symmetric and asymmetric equilibria, and their properties as a function of the structural parameters, while in the companion paper Cavalli et al. 2016 we focus on the best reply dynamics. Preliminary investigations show that the modelling approach we adopted allows for the occurrence of non trivial and complex dynamics.

The remainder of the paper is organized as follows. In Section 2 we present a general contest model and we introduce assumptions to specify several characteristics of the contest model we aim to investigate. Section 3 reports our main results, while proofs are in Appendix. Section 4 concludes.

\section{Modelling Contests}

In this section we propose a micro founded contest model starting from a general perspective. In the subsequent sections, we will introduce specific assumptions on these basic elements. This allows connecting the characteristics of the set of equilibria with the properties of these basic elements on the basis of transparent micro assumptions.

In contests, two or more contenders use available resources to try to hamper, disable and destroy the rival in order to reach their own aim. Hence, in any contest situation there are at least two agents, which from now on will be identified by part $i$ and $j$, who aim to achieve a particular goal. As a starting point, we will limit ourself just to two players. As discussed in Hirshleifer 1995a and 1995b, we agree that the incompatibility between agents' goals is the root of any contests: each part tries to reach the best possible approximation 
to its goal, and to do this, takes part in a conflict, trying to influence the probability of getting an outcome through a suitable effort. The distance between the goals represents the polarization of the contest situation, and we will show that it plays a crucial role. Each agent's effort can have several consequences. First, one must consider the effectivity of efforts, which encompasses and represents the different technological and institutional characteristics of each part. To model the possibility that a part can directly hamper or disable opponent's capability, we suppose that each agent's effectivity function might depend on both the amount of effort of $i$ and $j$. Moreover, the agents' efforts can affect its own and the opponents' goal too, so that, depending on the respective efforts, we can have that the goals can change. More generally, the outcome of the contest might depend on both agents' effort.

These considerations lead us to propose the following definition of a two agent contest model.

Definition 1. A two agent Contest Model (CM) can be described by a sextuple (X, $\mathbf{S}, \mathbf{C}, \boldsymbol{\zeta}, \mathbf{P}, \mathbf{U})$, in which each component is a vector of two elements such that

1. $\mathbf{X}=\left(X_{1}, X_{2}\right)$ is the set of agents' efforts $\left(x_{i}, x_{j}\right)$ such that

$$
x_{i} \in X_{i} \subseteq \mathbb{R}^{+}, i \in\{1,2\}
$$

2. $\mathbf{S}=\left(S_{1}, S_{2}\right)$ is a couple of functions $S_{i}\left(x_{i}, x_{j}\right)$

$$
S_{i}: X_{i} \times X_{j} \rightarrow \mathbb{R}^{+}, i \in\{1,2\}
$$

which represent the effectivity of agents' efforts, i.e. it measures the impact of $i^{\prime} s$ effort on the likelihood of getting a specific outcome. Note that it might be affected by opponent's effort too;

3. $\mathbf{C}=\left(C_{1}, C_{2}\right)$, is a couple of functions $C_{i}\left(x_{i}\right)$

$$
C_{i}: X_{i} \rightarrow \mathbb{R}^{+}, i \in\{1,2\}
$$

which represents the agents' cost function, 1

4. $\zeta=\left(\zeta_{1}, \zeta_{2}\right)$ is a couple of functions $\zeta_{i}\left(s_{i}, s_{j}\right)$

$$
\zeta_{i}: \mathbb{R}^{+} \times \mathbb{R}^{+} \rightarrow \mathbb{R}
$$

which represents $i^{\prime}$ s outcome function, describing the result of the contest for agent $i$ as a function of both agents' efforts' effectivity $\left(s_{i}, s_{j}\right)$;

5. $\mathbf{P}=\left(P_{1}, P_{2}\right)$, is a couple of conditional probability measures $P_{i}\left(s_{1}, s_{2}\right)$

$$
P_{i}: \mathbb{R}^{+} \times \mathbb{R}^{+} \rightarrow \Delta(\mathbb{R}) 2
$$

representing the contest success technology (CST) connecting agents' effectivity of the efforts $\left(s_{1}, s_{2}\right)$ to the probability of getting an outcome $z_{i} \in\left[\underline{g_{1}}, \overline{g_{2}}\right]$;

6. $\mathbf{W}=\left(W_{1}, W_{2}\right)$ is a couple of functions $W_{i}\left(\widetilde{z}_{i}\right)$

$$
W_{i}: \widetilde{Z}_{i} \rightarrow \mathbb{R}, i \in\{1,2\}
$$

which represents the agents' utility function evaluating random outcomes $\widetilde{z}_{i}$ distributed according to $P_{i}\left(s_{1}, s_{2}\right)$. As usual $W_{i}$ is supposed to satisfy expected utility representation, so that there exists a $U_{i}: \mathbb{R} \rightarrow \mathbb{R}$ such that

$$
W_{i}\left(\widetilde{z}_{i}\right)=\int U_{i}\left(z_{i}\right) d P_{i}\left(z_{i} \mid s_{1}, s_{2}\right)
$$

${ }^{1}$ We checked that spillover in the cost function would not add any interesting insights on equilibrium properties.

${ }^{2}$ As usual $\Delta(\cdot)$ denotes the set of all probability measure on the set $\cdot$ 
The model presented in Definition 1 is very general and can be applied to any contest situation. Although apparently differs from the basic model used in contest theory 3 , it is quite immediate to show that the model we propose falls within the general definition of contest. Usually a contest is defined by the following elements.

1. A finite set of agents, the contenders

$$
N=\{1, \ldots, n\}
$$

2. A set of possible actions taken by the agents before a prize is allocated

$$
a_{i} \in A_{i}
$$

3. A prize that may depend on agents' actions

$$
V_{i}: A_{1} \times \ldots \times A_{n} \rightarrow \Omega_{i} \subseteq \mathbb{R}
$$

4. A contest success function relating agents' actions to the agent's probability of obtaining the prize

$$
p_{i}: A_{1} \times \ldots \times A_{n} \rightarrow \Delta\left(\Omega_{i}\right) ;
$$

5. A utility function evaluating each agent prize

$$
U_{i}: \Omega_{i} \rightarrow \mathbb{R}
$$

6. A cost function relating agents' actions to the cost of the actions

$$
C_{i}: A_{i} \rightarrow \mathbb{R}
$$

Then it is immediate that a CM is just a specific contest.

\section{Remark 1. Any CM is a contest.}

This remark might suggest that our definition is redundant. However, we consider more effective to start from the elements introduced by Definition 1 The reason is that we believe that our model allows a more neat analysis, allowing a better match with real situations and a more immediate interpretation and understanding of the theoretic results. More generally, our model allows a discussion of the micro foundations of the peculiar functional forms we consider for our concepts, a discussion that is not immediate for many standard contest models, often a sort of reduced form model.

Before providing assumptions to focus on a particular class of CM, we recall that the agent's effort is its choice variable, that affects the probability of reaching an outcome once transformed by function $S_{i}$, representing the effectivity of the effort. The role of this function is to separate the agents' effort effectivity, which is part of $i$ 's characteristics, from the probability of getting a specific outcome, the standard contest success technology. In particular, our formulation allows for introducing and studying the effect of spillover in any constitutive element of a contest, in particular in effectivity and outcomes. This will be the main goal of Sections 3.2 and 3.3, in which we will show the consequences of such possible spillover on existence and multiplicity of equilibria. We will show that spillover in different components of a CM have different effects on the set of equilibria. Hence the characteristics of the set of equilibria are connected to the structural properties of the $\mathrm{CM}$, i.e. on the specific historical, institutional and geographical conditions. Finally, note that if the agents' outcomes $\zeta_{i}$ do not depend on the players' efforts, then the model belongs to the class of rent seeking models, while in the models of production and conflict the value of the goals is endogenously determined by the agents' choices on how much time is used to conflict and consequently on production 4

\footnotetext{
${ }^{3}$ See for example Corchon 2007 or Konrad 2009.

${ }^{4}$ See e.g. Garfinkel and Skaperdas 2007, Hausken 2005, Konrad 2009, Neary 1997.
} 


\subsection{Restriction on the Contest Model}

In this section, we introduce, and then discuss, a set of assumptions on the CM to generate a family of contests sufficiently general to describe a suitably wide range of situations, but, at the same time, suitably specialized to allow deriving significant results. Introducing progressive restrictive assumptions also allows us to better understand the role and the relation between the structural characteristics of the elements of a $\mathrm{CM}$ and the properties of the set of equilibria of the associated game.

\section{Assumption 1. GENERAL ASSUMPTIONS ON THE CONTEST MODEL:}

1. SYMMETRIC CONTEST MODEL: all the components of a CM are symmetric

$$
\begin{gathered}
X_{i}=X_{j}, S_{i}\left(x_{i}, x_{j}\right)=S_{j}\left(x_{j}, x_{i}\right), C_{i}\left(x_{i}, x_{j}\right)=C_{j}\left(x_{j}, x_{i}\right), \\
\zeta_{i}\left(s_{i}, s_{j}\right)=-\zeta_{j}\left(s_{j}, s_{i}\right), P_{i}\left(s_{i}, s_{j}\right)=P_{j}\left(s_{j}, s_{i}\right), U_{i}=U_{j} .
\end{gathered}
$$

2. UPPER BOUND FOR EFFORT: the set of each agent's effort $X_{i} \subseteq \mathbb{R}^{+}$is compact. Hence, because of assumption 1, w.l.g. we pose

$$
X_{i}=[0,1] .
$$

3. SMOOTH CONTEST MODEL: all the functions involved in a $C M, S_{i}\left(x_{i}, x_{j}\right), g_{i}\left(x_{i}, x_{j}\right), C_{i}\left(x_{i}\right)$, $\zeta_{i}\left(s_{i}, s_{j}\right), P_{i}\left(s_{i}, s_{j}\right), U_{i}\left(\zeta_{i}\right)$ are twice continuously differentiable.

4. POSSIBLE OUTCOMES: for each player, the interaction might end in two ways, either reaching its goal $g_{i}\left(x_{i}, x_{j}\right)$ or its defeat outcome $d_{i}\left(x_{i}, x_{j}\right)$ :

$$
\forall\left(s_{i}, s_{j}\right) \in \mathbb{R}^{+} \times \mathbb{R}^{+} \quad \zeta_{i}\left(s_{i}, s_{j}\right) \in\left\{g_{i}\left(x_{i}, x_{j}\right), d_{i}\left(x_{i}, x_{j}\right)\right\} \subseteq\left[\underline{g_{1}}, \overline{g_{2}}\right],
$$

where

$$
g_{i}: X_{i} \times X_{j} \rightarrow\left[\underline{g_{i}}, \overline{g_{i}}\right] \subseteq \mathbb{R}, \quad d_{i}: X_{i} \times X_{j} \rightarrow\left[\underline{g_{1}}, \overline{g_{2}}\right] \subseteq \mathbb{R}:
$$

without loss of generality, we denote by $\underline{g_{i}}$ and $\overline{g_{i}}$, respectively, the inferior and the upper bounds of $i$ 's outcomes, so that $\underline{g_{i}} \leq \overline{g_{i}}$ and $\overline{g_{1}} \leq 0 \leq \underline{g_{2}}$;

5. RATIO CONTEST SUCCESS TECHNOLOGY: the CST $P_{i}\left(s_{i}, s_{j}\right)$ has a ratio form 5 Hence, given previous assumption 4 , we get

$$
P_{i}\left(z_{i} \mid S_{i}\left(x_{i}, x_{j}\right), S_{j}\left(x_{j}, x_{i}\right)\right)= \begin{cases}\frac{S_{i}\left(x_{i}, x_{j}\right)}{S_{i}\left(x_{i}, x_{j}\right)+S_{j}\left(x_{j}, x_{i}\right)} & \text { if } z_{i}=g_{i}\left(x_{i}, x_{j}\right), \\ \frac{S_{j}\left(x_{j}, x_{i}\right)}{S_{i}\left(x_{i}, x_{j}\right)+S_{j}\left(x_{j}, x_{i}\right)} & \text { if } z_{i}=d_{i}\left(x_{i}, x_{j}\right), \\ 0 & \text { otherwise. }\end{cases}
$$

6. AGENTS' UTILITY FUNCTIONS: each agent's utility function $U_{i}$ is decreasing in the distance $\left|z_{i}-g_{i}\left(x_{i}, x_{j}\right)\right|$ between $i$ 's outcome and its goal.

7. POSSIBLE SPILLOVER: the model allows for possible specific spillover in the components of a $C M$

$$
\begin{gathered}
\frac{\partial S_{i}\left(x_{i}, x_{j}\right)}{\partial x_{j}} \leq 0, \\
\frac{\partial g_{1}}{\partial x_{1}} \leq 0, \frac{\partial g_{1}}{\partial x_{2}} \leq 0, \frac{\partial d_{1}}{\partial x_{2}} \geq 0, \frac{\partial d_{1}}{\partial x_{1}} \geq 0, \\
\frac{\partial g_{2}}{\partial x_{2}} \geq 0, \frac{\partial g_{2}}{\partial x_{1}} \geq 0, \frac{\partial d_{2}}{\partial x_{1}} \leq 0, \frac{\partial d_{2}}{\partial x_{2}} \leq 0 .
\end{gathered}
$$

In the next paragraphs we detail and explain each point of Assumption 1

\footnotetext{
${ }^{5}$ This is the terminology used by Hirshleifer 1989, an alternative is to call this contest success technology logit.
} 


\subsubsection{Symmetry}

The reason for the symmetry assumption is trivially that in the present paper we aim to study a symmetric contest. In this way we assure that the results we find about equilibria characteristics only depend on structural parameters and not on players' asymmetry. Moreover, it is interesting to understand whether and when asymmetric equilibria can arise in symmetric contests, clarifying the structural assumptions that generate such a situation. Finally, notice that symmetry implies

$$
g_{i}\left(x_{i}, x_{j}\right)=-g_{j}\left(x_{j}, x_{i}\right) \text { and } d_{i}\left(x_{i}, x_{j}\right)=-d_{j}\left(x_{j}, x_{i}\right) .
$$

\subsubsection{The Efforts set}

We assume that contestants are exogenously constrained regarding the amount of effort they can choose. The reasons for this assumption are two. First, we believe it is hardly realistic to consider an unbounded amount of effort, secondly, this assumption allows interpreting agents' bids as intensity, i.e. as the percentage of the stock of the available resources used for the contest game. This assumption is crucially important to allow for the occurrence of corner equilibria, which have obvious significant interpretations: once we interpret bids as intensity of effort, then the results on interior or corner equilibria have a more clear counterpart in actual contest situations as null or intermediate or maximum effort.

\subsubsection{The Possible Outcomes}

We restrict the set of $i$ 's possible outcomes to just two possibilities: either $i$ reaches its goal $g_{i}$ or it get a defeat outcome $d_{i}$. This simplified framework is sufficient to derive our main results and allows identifying specific parameters and functions that plays a crucial role for our results and that have clear counterparts in real situations. Note that even if in Assumption 1 we allow for any kind of direct effect of players' bids on players' goal and defeat outcomes, in the next subsection we restrict the analysis to particular, significant spillover.

\subsubsection{Smoothness and Contest Technology}

The smoothness requirement, which might seem a neutral technical assumption, actually has substantial implications, for example in the choice of the possible contest success technology. The first consequence is that it excludes the common Tullock contest technology 6 which is not continuously differentiable in $(0,0)$.

Consider the assumptions of continuous differentiable functions ratio CT with respect to the classic ratio technology as axiomatized in Skaperdas 1996. Since our technology is a ratio CST, it satisfies Skaperda's axioms 1 to 5 that characterize such functional form. However, it does not satisfy the homogeneity axiom A6

which implies

$$
\frac{S\left(\lambda x_{i}, \lambda x_{j}\right)}{S\left(\lambda x_{i}, \lambda x_{j}\right)+S_{j}\left(\lambda x_{j}, \lambda x_{i}\right)}=\frac{S\left(x_{i}, x_{j}\right)}{S\left(x_{i}, x_{j}\right)+S\left(x_{j}, x_{i}\right)},
$$

$$
\frac{S\left(x_{i}, x_{j}\right)}{S\left(x_{i}, x_{j}\right)+S\left(x_{j}, x_{i}\right)}= \begin{cases}\frac{1}{2} & \text { for } x_{i}=x_{j} \in[0,1], \\ 1 & \text { for } x_{i} \in(0,1], x_{j}=0 .\end{cases}
$$

This means that a CST satisfying the homogeneity axiom can't be continuous in $(0,0)$. Indeed, this axiom is motivated by the idea that the probability of reaching the goal should be independent of units of measurement. However, because of the assumption of compactness of the effort sets and thus of the interpretation of bids

\footnotetext{
${ }^{6}$ Tullock 1967 and 1980.
} 
as intensity of effort, axiom A6 is no more relevant. The homogeneity axiom, together with axioms 1 to 5 , has the effect to restrict the set of possible CST to the following generalization of Tullock technology

$$
T\left(x_{i}, x_{j}\right)= \begin{cases}\frac{x_{i}^{\beta}}{x_{i}^{\beta}+x_{j}^{\beta}} & \text { if } \quad\left(x_{i}, x_{j}\right) \neq(0,0), \\ \frac{1}{2} & \text { if }\left(x_{i}, x_{j}\right)=(0,0) .\end{cases}
$$

Noticing that

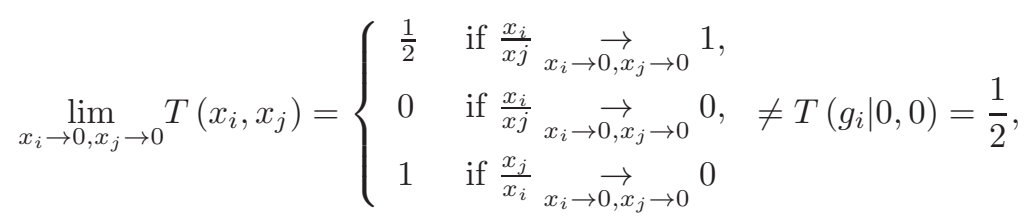

and that

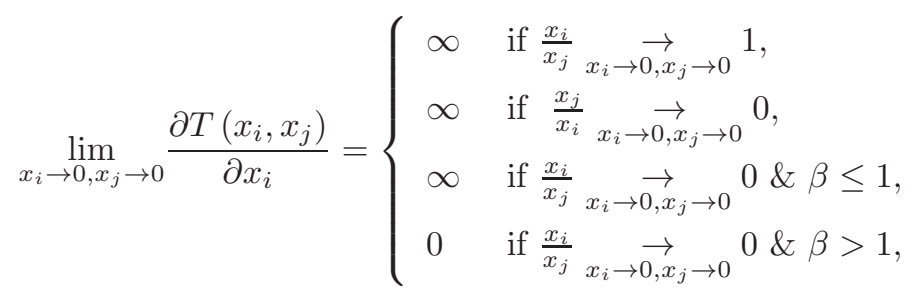

which means that a marginal increment in $i$ 's effort has a huge effect on the marginal probability of getting $z_{i}$. Such discontinuities have the unpleasant consequence of preventing the occurrence of zero equilibria. Instead, we assume continuously differentiable functions, so that our CST has a symmetric and intuitive behavior of the marginal probability of getting $z_{i}$ in a neighborhood of zero effort too. This allows zero effort equilibria for a suitable combination of structural parameters.

Finally, notice that even if the logistic CST

$$
L\left(x_{i}, x_{j}\right)=\frac{e^{\beta x_{i}}}{e^{\beta x_{i}}+e^{\beta x_{j}}}
$$

is a smooth function, it has however the uncomfortable property of admitting either no pure strategy equilibria or zero effort equilibria 8 Moreover, the structural parameters that induce such kind of equilibria have no clear micro founded meaning.

In Section 3, we will propose and use a different smooth ratio CT and our results will show that, together with the other assumptions, it avoids these unpleasant equilibrium properties.

\subsubsection{The Utility Function}

We explicitly introduce a utility function defined on the set of possible outcomes in order to have a micro founded contest model that clarifies the links between equilibrium properties and structural characteristics. We remark that even if the specific form of utility function we consider is quite common in the political economy literature 9 its use in contest theory is new and has important implications. In particular, it allows the introduction of auxiliary concepts that help to interpret our results. Note that these preferences are single peaked and

$$
\arg \max U_{i}\left(z_{i}\right)=g_{i}\left(x_{i}, x_{j}\right)
$$

i.e. $i$ 's goal is its bliss point, which reinforces our interpretation of $g_{i}\left(x_{i}, x_{j}\right)$. Using this property, it is natural to define a contest polarization as the distance between agents' bliss points.

\footnotetext{
${ }^{7}$ See e.g. Corchon 2007 and Konrad 2009.

${ }^{8}$ See Baik 1988.

${ }^{9}$ See for example Persson - Tabellini 2000.
} 
Definition 2. The measure of contest polarization is defined through the function $\rho: \mathbb{R}^{+} \times \mathbb{R}^{+} \rightarrow \mathbb{R}^{+}$given by

$$
\rho\left(x_{1}, x_{2}\right):=g_{2}\left(x_{2}, x_{1}\right)-g_{1}\left(x_{1}, x_{2}\right) .
$$

The reason to use the distance between agents' goals as a measure of polarization is the obvious intuition that the more the agents' goals diverge, the higher they become involved in the contest, as argued in Hirshleifer 1991, 1995a, 1995b and in Hirshleifer and Osborne 2001. In particular, note that agents' goal incompatibility is equivalent to strictly positive polarization. We will show that the way the polarization behaves is one of the crucial elements to understand equilibrium behavior in contests: since by construction the agents' goals are incompatible, i.e.

$$
\underline{g_{2}} \geq \overline{g_{1}} \Leftrightarrow \rho\left(x_{1}, x_{2}\right)>0 \forall\left(x_{i}, x_{j}\right),
$$

the attempt to reach the best possible outcome leads to compete. This is where the source of the conflicting behavior is encompassed in our contest model. The utility function we use leads to a payoff function that depends on both the polarization $\rho\left(x_{1}, x_{2}\right)$ and the defeat outcome $d_{i}\left(x_{i}, x_{j}\right)$

$$
\begin{aligned}
U_{i}\left(z_{i}\right) & =f_{i}\left(\left|z_{i}-g_{i}\left(x_{i}, x_{j}\right)\right|\right) \\
& =\frac{S_{i}\left(x_{i}, x_{j}\right)}{S_{i}\left(x_{i}, x_{j}\right)+S_{j}\left(x_{j}, x_{i}\right)} f_{i}(0)+\frac{S_{j}\left(x_{j}, x_{i}\right)}{S_{i}\left(x_{i}, x_{j}\right)+S_{j}\left(x_{j}, x_{i}\right)} f_{i}\left(\left|d_{i}\left(x_{i}, x_{j}\right)-g_{i}\left(x_{i}, x_{j}\right)\right|\right) \\
& =\frac{S_{j}\left(x_{j}, x_{i}\right)}{S_{i}\left(x_{i}, x_{j}\right)+S_{j}\left(x_{j}, x_{i}\right)} f_{i}\left(\rho\left(x_{1}, x_{2}\right)-\left|g_{j}\left(x_{i}, x_{j}\right)-d_{i}\left(x_{i}, x_{j}\right)\right|\right) .
\end{aligned}
$$

\subsubsection{The Possible Spillover}

Our CM allows for direct spillover from the counterpart's choices in the basic functions of the model, the effectivity and the outcomes. Now we introduce and discuss three possible assumptions about the characteristics of these spillover, assumptions that we will explore in the following sections.

Assumption 2. THE POLARIZATION ASSUMPTION: the greater each player's effort is, the greater the polarization becomes, while opponent's effort has no effect on goals:

$$
\frac{\partial g_{1}}{\partial x_{1}}<0, \frac{\partial g_{1}}{\partial x_{2}}=0, \frac{\partial g_{2}}{\partial x_{2}}>0, \frac{\partial g_{2}}{\partial x_{1}}=0
$$

Then, from now on we will write

$$
g_{1}\left(x_{1}\right):=g_{1}\left(x_{1}, x_{2}\right) \text { and } g_{2}\left(x_{2}\right):=g_{2}\left(x_{2}, x_{1}\right)
$$

The idea behind this assumption is that an agent's own effort's intensity push its own goal away from the opponent's one. In particular the polarization assumption has the crucial implication that the contest polarization increases with both agents' intensity of efforts: since

$$
\rho\left(x_{1}, x_{2}\right)=g_{2}\left(x_{2}\right)-g_{1}\left(x_{1}\right)
$$

then

$$
\frac{\partial \rho\left(x_{1}, x_{2}\right)}{\partial x_{1}}=-\frac{\partial g_{1}\left(x_{1}\right)}{\partial x_{1}}>0 \text { and } \frac{\partial \rho\left(x_{1}, x_{2}\right)}{\partial x_{2}}=\frac{\partial g_{2}\left(x_{2}\right)}{\partial x_{2}}>0 .
$$

We believe that this is common to many settings where the intensity of contestants' behavior increases the distance between players' goals.

Assumption 3. THE RADICALIZATION ASSUMPTION: the greater each player's effort is, the worse the opponent's defeat outcome:

$$
\frac{\partial d_{2}}{\partial x_{1}}<0, \frac{\partial d_{2}}{\partial x_{2}}=0, \frac{\partial d_{1}}{\partial x_{2}}>0, \frac{\partial d_{1}}{\partial x_{1}}=0 .
$$


Then, from now on we will write

$$
d_{1}\left(x_{2}\right):=d_{1}\left(x_{1}, x_{2}\right) \text { and } d_{2}\left(x_{1}\right):=d_{2}\left(x_{2}, x_{1}\right)
$$

The idea behind this assumption is that an agent's effort affects the counterpart's defeat outcome, i.e. the greater the intensity of effort, the worse the defeat conditions. In particular the radicalization assumption implies that the counterpart's effort intensity push the defeat outcome away from a player's bliss point, so that

$$
\frac{\partial\left[d_{1}\left(x_{2}\right)-g_{1}\left(x_{1}\right)\right]}{\partial x_{2}}=\frac{\partial d_{1}\left(x_{2}\right)}{\partial x_{2}}>0 \text { and } \frac{\partial\left[g_{2}\left(x_{2}\right)-d_{2}\left(x_{1}\right)\right]}{\partial x_{1}}=-\frac{\partial d_{2}\left(x_{2}\right)}{\partial x_{2}}>0 .
$$

Again, we believe that this case where an increase of a contestant's effort induces a worse outcome for the loser is quite common and characterizes situations where the confrontation between counterparts lead to a more radical standing towards the loser of the contest.

Assumption 4. THE DIRECT DESTRUCTIVE ASSUMPTION: the greater each player effort is, the lower the effectivity of the counterpart's effort becomes, i.e.

$$
\frac{\partial S_{i}\left(x_{i}, x_{j}\right)}{\partial x_{j}}<0
$$

The idea behind this assumption is that an agent's effort can directly reduce the effectivity of the counterpart's effort, for physical, economic or institutional reasons. Note that the expected effects on the CST are reinforced by the direct destructive assumption:

1. The probability of getting its goal is increasing in an agent effort

$$
\frac{\partial P_{i}\left(g_{i} \mid S_{i}, S_{j}\right)}{\partial x_{i}}=\frac{\frac{\partial S_{i}\left(x_{i}, x_{j}\right)}{\partial x_{i}} S_{j}\left(x_{j}, x_{i}\right)-\frac{\partial S_{j}\left(x_{i}, x_{j}\right)}{\partial x_{i}} S_{i}\left(x_{i}, x_{j}\right)}{\left[S_{i}\left(x_{i}, x_{j}\right)+S_{j}\left(x_{j}, x_{i}\right)\right]^{2}}>0 ;
$$

2. the probability of getting the defeat outcome is increasing in the opponent's effort

$$
\frac{\partial P_{i}\left(d_{i} \mid S_{i}, S_{j}\right)}{\partial x_{j}}=\frac{\frac{\partial S_{j}\left(x_{i}, x_{j}\right)}{\partial x_{j}} S_{i}\left(x_{j}, x_{i}\right)-\frac{\partial S_{i}\left(x_{i}, x_{j}\right)}{\partial x_{j}} S_{j}\left(x_{i}, x_{j}\right)}{\left[S_{i}\left(x_{i}, x_{j}\right)+S_{j}\left(x_{j}, x_{i}\right)\right]^{2}}>0 .
$$

The results of the next sections will show that these different possible assumptions play a different role generating different characteristics of the equilibrium sets.

\subsection{The Associated Game}

From the definition of CM it is easy to derive a payoff function $\pi_{i}: X_{i} \times X_{j} \rightarrow \mathbb{R}$ as

$$
\begin{aligned}
\pi_{i}\left(x_{i}, x_{j}\right) & =\int_{\underline{g}_{1}}^{\bar{g}_{2}} U_{i}\left(z_{i}\right) d P_{i}\left(z_{i} \mid S_{i}\left(x_{i}, x_{j}\right), S_{j}\left(x_{i}, x_{j}\right)\right)-C_{i}\left(x_{i}\right) \\
& =\frac{S_{j}\left(x_{j}, x_{i}\right)}{S_{i}\left(x_{i}, x_{j}\right)+S_{j}\left(x_{j}, x_{i}\right)} f_{i}\left(\left|d_{i}\left(x_{j}\right)-g_{i}\left(x_{i}\right)\right|\right)-C_{i}\left(x_{i}\right) \\
& =\frac{S_{j}\left(x_{j}, x_{i}\right)}{S_{i}\left(x_{i}, x_{j}\right)+S_{j}\left(x_{j}, x_{i}\right)} f_{i}\left(\rho\left(x_{1}, x_{2}\right)-\left|g_{j}\left(x_{j}\right)-d_{i}\left(x_{j}\right)\right|\right)-C_{i}\left(x_{i}\right)
\end{aligned}
$$

and thus we obtain the class of associated strategic form game $\Gamma^{C M}=\left\{\Gamma=\left(\{1,2\},[0,1]^{2}, \pi_{i}\left(x_{i}, x_{j}\right)\right)\right\}$.

We recall the definition of symmetric game. 
Definition 3. A two players strategic form game

$$
\Gamma=\left(\{1,2\}, X_{1} \times X_{2}, \pi_{1}\left(x_{1}, x_{2}\right), \pi_{2}\left(x_{2}, x_{1}\right)\right) .
$$

is symmetric if and only if

$$
\pi_{1}\left(x_{1}, x_{2}\right)=\pi_{2}\left(x_{1}, x_{2}\right), \forall x_{i} \in X_{i}, i=1,2
$$

\section{Remarks}

1. The strategic form game associated to a symmetric CM is symmetric;

2. The best reply correspondences

$$
B R_{i}\left(x_{j}\right):=\underset{x_{i} \in X_{i}}{\arg \max } \pi_{i}\left(x_{i}, x_{j}\right)
$$

of a two players symmetric strategic form game are symmetric, i.e.

$$
B R_{i}\left(x_{j}\right):=B R_{j}\left(x_{i}\right)
$$

\section{$3 \quad$ The Results}

In this section we aim at studying different possible sets of equilibria arising in the symmetric contest model defined by Assumption 1. To this end, to better understand the role of each assumption on the characterization of the equilibria set and to keep each case analytically tractable, we simplify as much as possible the expression of each involved function, so that each parameter has a clear interpretation. After specifying the family of CM we aim to investigate, we focus on three different scenarios, characterized by specific spillover effects. In particular, we are interested in studying the cases in which there is

- no spillover;

- spillover on outcomes, stressing the different role of the polarization and of the radicalization assumption;

- spillover in contest success technology (direct destructive assumption).

For each scenario we provide the expressions of the best response correspondences and the possible sets of Nash equilibria. In particular, we classify best response correspondences with respect to their monotonicity. To this end, we remark that a strictly non-increasing (respectively non-decreasing) function is a non-increasing (respectively non-decreasing) but non-constant function, while a hump-shaped function on an interval $\left[x_{1}, x_{2}\right]$ is strictly non-decreasing on $\left[x_{1}, c\right]$ and strictly non-increasing on $\left[c, x_{2}\right]$, where $c \in\left(x_{1}, x_{2}\right)$.

We assume that all the functional forms in the elements defining a CM are linear. We notice that, despite the simplification, linear functions can be seen as a local approximation of smooth functions 10

\section{Assumption 5. LINEAR CONTEST MODEL}

\section{BILINEAR EFFECTIVITY FUNCTIONS:}

$$
S_{i}\left(x_{i}, x_{j}\right)=\beta x_{i}\left(1-\alpha x_{j}\right)+1
$$

where $\beta>0$ and $\alpha \in[0,1]$, so that $S_{i}$ is linear in $x_{i}$ and the marginal productivity of $i$ 's effort is linearly decreasing in $x_{j}$. We notice that when the direct destructive assumption holds, we have $\alpha>0$, otherwise $\alpha=0$.

\footnotetext{
${ }^{10}$ Dasgupta and Nti 1998 provide an alternative justification to focus on linear functions in contest models.
} 


\section{LINEAR OUTCOMES:}

$$
g_{1}\left(x_{1}\right)=-\theta-\delta x_{1}, \quad d_{1}\left(x_{2}\right)=\gamma x_{2}, \quad g_{2}\left(x_{2}\right)=\theta+\delta x_{2}, \quad d_{2}\left(x_{1}\right)=-\gamma x_{1},
$$

where $\theta>0$ and $\delta$ and $\gamma$ are both non negative constants. We notice that when the radicalization assumption holds, we have $\delta>0$ and $\gamma>0$, otherwise $\delta=\gamma=0$.

\section{LINEAR UTILITY FUNCTION:}

$$
U_{1}(z)=-\left(z-g_{1}\left(x_{1}\right)\right), \quad U_{2}(z)=-\left(g_{2}\left(x_{2}\right)-z\right),
$$

where w.l.g. we normalize each linear function.

\section{LINEAR COST FUNCTIONS WITHOUT SPILLOVER:}

$$
C_{i}\left(x_{i}\right)=x_{i}
$$

where w.l.g. we assume unitary marginal cost.

The previous assumptions imply the following CST function

$$
P_{i}\left(z_{i} \mid S_{i}\left(x_{i}, x_{j}\right), S_{j}\left(x_{j}, x_{i}\right)\right)= \begin{cases}\frac{\beta x_{i}\left(1-\alpha x_{j}\right)+1}{\beta x_{i}\left(1-\alpha x_{j}\right)+\beta x_{j}\left(1-\alpha x_{i}\right)+2} & \text { if } z_{i}=g_{i}\left(x_{i}\right), \\ \frac{\beta x_{j}\left(1-\alpha x_{i}\right)+1}{\beta x_{i}\left(1-\alpha x_{j}\right)+\beta x_{j}\left(1-\alpha x_{i}\right)+2} & \text { if } z_{i}=d_{i}\left(x_{j}\right), \\ 0 & \text { otherwise, }\end{cases}
$$

so that the resulting payoff functions of the strategic form game associated to the linear CM are

$$
\pi_{i}\left(x_{i}, x_{j}\right)=-\frac{\beta x_{j}\left(1-\alpha x_{i}\right)+1}{\beta x_{i}\left(1-\alpha x_{j}\right)+\beta x_{j}\left(1-\alpha x_{i}\right)+2}\left[\theta+\delta x_{i}+\gamma x_{j}\right]-x_{i} .
$$

In this way, we obtain the class of strategic form game $\Gamma^{L}$, where $\pi_{i}$ are defined by (2). Notice that the constant term " +1 " in the expression of the effectivity function, thanks to the CST function, is absolutely general. In fact, the case of $S_{i}\left(x_{i}, x_{j}\right)=\beta x_{i}\left(1-\alpha x_{j}\right)+k$ with $k>0$ can be easily rephrased in the present

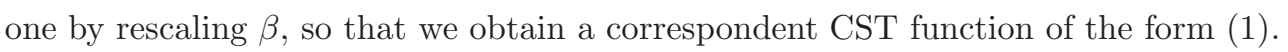

Let now discuss the meaning of parameters and functions and their role in the linear CM.

Firstly, we focus on the CST. Apart from continuity, the CST we consider has two important characteristics. First, as with Tullock's CST, there is equiprobability of both outcomes when both agents bid the same intensity of effort

$$
x_{j}=x_{i}=x^{*} \Leftrightarrow P_{i}\left(z_{i} \mid S_{i}, S_{j}\right)= \begin{cases}\frac{\beta x^{*}\left(1-\alpha x^{*}\right)+1}{\beta x^{*}\left(1-\alpha x^{*}\right)+\beta x^{*}\left(1-\alpha x^{*}\right)+2}=\frac{1}{2} & \text { if } z_{i}=g_{i}, \\ \frac{\beta x^{*}\left(1-\alpha x^{*}\right)+1}{\beta x^{*}\left(1-\alpha x^{*}\right)+\beta x^{*}\left(1-\alpha x^{*}\right)+2}=\frac{1}{2} & \text { if } z_{i}=d_{i}, \\ 0 & \text { otherwise. }\end{cases}
$$

However, differently from Tullock's CST, the present CST does never reach probability 1 even when there is full asymmetry of efforts

$$
P_{i}\left(z_{i} \mid S_{i}(1,0), S_{j}(0,1)\right)= \begin{cases}\frac{\beta+1}{\beta+2} & \text { if } z_{i}=g_{i}(1) \\ \frac{1}{\beta+2} & \text { if } z_{i}=d_{i}(0) \\ 0 & \text { otherwise }\end{cases}
$$




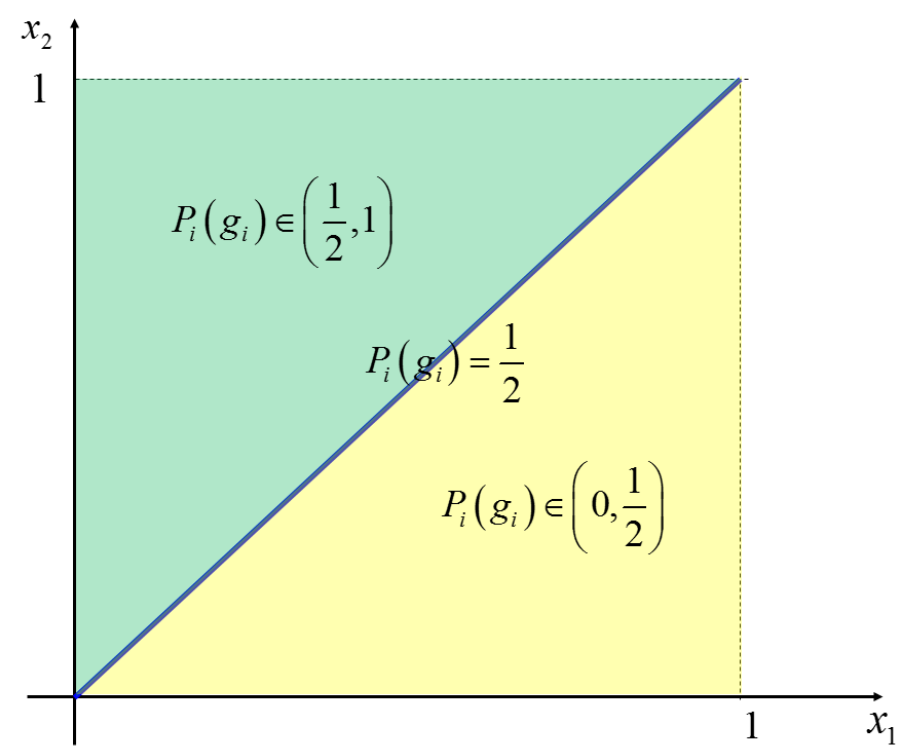

Figure 1: Probability of achieving goal depending players' efforts.

i.e. it is as if there is an implicit noise such that no player can win with certainty even if it deploys the maximum intensity against null counterpart's intensity. Nevertheless, as $\beta$ increases, the probability of success converges toward 1, as well as that of defeat vanishes. The situation is illustrated in Figure 1 .

Counter-intuitive consequences of the use of the generalized Tullock CST are not restricted to the occurrence of discontinuities, also the interpretation of $\beta$ as a measure of the marginal impact of an increase in an agent's effort is debatable. Actually while the derivative of the effectivity function $x_{i}^{\beta}$ is increasing or decreasing in $x$ according to $\beta \gtrless 1$ and it is not monotone with respect to $\beta$, in our case $\beta$ is trivially the marginal productivity of $i$ 's effort on its effectivity function and has the intuitive properties we expect from marginal productivity. Since

$$
\frac{\partial S_{i}\left(x_{i}, x_{j}\right)}{\partial x_{i}}=\beta\left(1-\alpha x_{j}\right)
$$

is obviously constant in $x_{i}$, decreasing with respect to the counterpart's effort $x_{j}$ when there are spillover and monotonically increasing in $\beta$, then $\beta$ is an obvious measure of the marginal productivity of effort on effectivity. To the best of our knowledge, the functional form for the agents' effectivity function we use here first appears in Dasgupta and Nti 1998. However, they approach the contest problem from a completely different point of view, since their aim is to support specific contest success functions, such the one used in this paper, from a mechanism design perspective. However, their results provide an independent justification for the use of this effectivity functional form.

Second, we remark that an obvious effect of the assumption on outcomes is that polarization now is linearly increasing in both agents' efforts

$$
\rho\left(x_{1}, x_{2}\right)=2 \theta+\delta\left(x_{1}+x_{2}\right) .
$$

Moreover, also the defeat outcomes are pushed toward a worse outcome by the counterpart's effort when there are spillover. Both these effects negatively affect $i$ 's expected outcome in the associated game's payoffs.

The parameters of this linear version of the CM are crucial for our results, hence to help the reader and the interpretation we report their meaning in the following table 


\begin{tabular}{|c|c|}
\hline Parameters & Meaning \\
\hline$\alpha \in[0,1]$ & destructiveness of effort \\
\hline$\beta \in(0,+\infty)$ & productivity of effort \\
\hline$\gamma \in[0,+\infty)$ & endogenous radicalization \\
\hline$\delta \in[0,+\infty)$ & endogenous polarization \\
\hline$\theta \in(0,+\infty)$ & ex ante polarization \\
\hline
\end{tabular}

Table 1: the structural parameters of the CM and their meaning

In the remainder of this section we are going to consider three families of games belonging to the class of strategic form game $\Gamma^{L}$, obtained when no spillover effects are taken into account, $\boldsymbol{\Gamma}^{L N S}$, when there are spillover on outcomes, $\Gamma^{L S O}$, and when spillover affects effectivity function, $\Gamma^{L S O}$.

\subsection{The case of no spillover}

This first example we consider is the simplest situation in which we have no spillover, which means setting $\alpha=\gamma=\delta=0$, so that $\Pi^{N S}=\beta \theta$ and $\Delta^{N S}=0$. The players' payoff functions then result

$$
\pi_{i}\left(x_{i}, x_{j}\right)=-\frac{\beta x_{j}+1}{\beta x_{i}+\beta x_{j}+2} \theta-x_{j},
$$

from which we have the class of strategic form games $\Gamma^{L N S}=\left\{\Gamma=\left(\{1,2\},[0,1]^{2}, \pi\right)\right\}$ with $\pi\left(x_{i}, x_{j}\right)=$ $\pi_{1}\left(x_{1}, x_{2}\right) \times \pi_{2}\left(x_{2}, x_{1}\right)$, where $\pi_{i}$ are defined by (8). Firstly we characterize the best response functions for this class of games.

Proposition 1. The best response functions related to games $\Gamma \in \Gamma^{L N S}$ are continuous, piecewise smooth functions given by

$$
B R_{i}\left(x_{j}\right)=\max \left\{\min \left\{-x_{j}-\frac{2}{\beta}+\frac{1}{\beta} \sqrt{\beta \theta\left(\beta x_{j}+1\right)}, 1\right\}, 0\right\},
$$

which satisfies $B R_{i}^{\prime}\left(x_{j}\right)=0$ for those $x_{j}$ for which $B R_{i}\left(x_{j}\right)=x_{j}$. Note that the best response function can be either constantly equal to 0, constantly equal to 1, strictly non-decreasing or hump-shaped.

The dependency of the possible best response function shapes from the parameter configurations is reported in Figure 2, We notice that by means of monotonicity of the best response functions we can actually classify corresponding games $\Gamma \in \Gamma^{L N S}$. In fact, when functions $B R_{i}$ are constantly equal to either 0 or 1 , we can say that game $\Gamma$ is characterized by strategic dominance, while when $B R_{i}$ is strictly non-decreasing, game $\Gamma$ is characterized by strategic complementarity.

Concerning the possible resulting equilibria, a key role is played by the continuity of best response functions and by their flatness at fixed points. The former aspect guarantees the existence of the Nash equilibrium while the latter one, together with continuity, provides uniqueness. We have the following Proposition.

Proposition 2. When the best response function (5) for games $\Gamma \in \Gamma^{L N S}$ is

- strictly non-decreasing, then the unique Nash equilibrium is a symmetric corner equilibrium;

- hump-shaped, then the unique Nash equilibrium is internal and symmetric;

- constant, then the unique Nash equilibrium is a symmetric corner equilibrium.

For any parameter configuration, no asymmetric equilibria occur. 


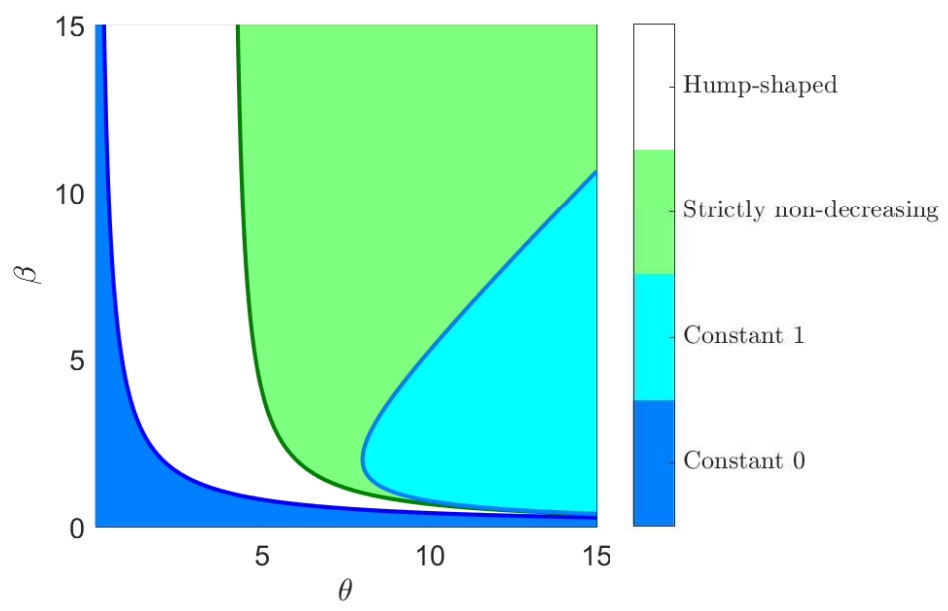

Figure 2: Possible best response functions of games $\Gamma \in \Gamma^{L N S}$ with respect to $\theta$ and $\beta$. Blue (respectively cyan) region represents parameters' combinations for which the best response is constantly equal to 1 (respectively to 0). Green (respectively white) region represents parameters' combinations for which the best response function is strictly non-decreasing (respectively hump-shaped). Darker shades of each color are used for region boundaries.
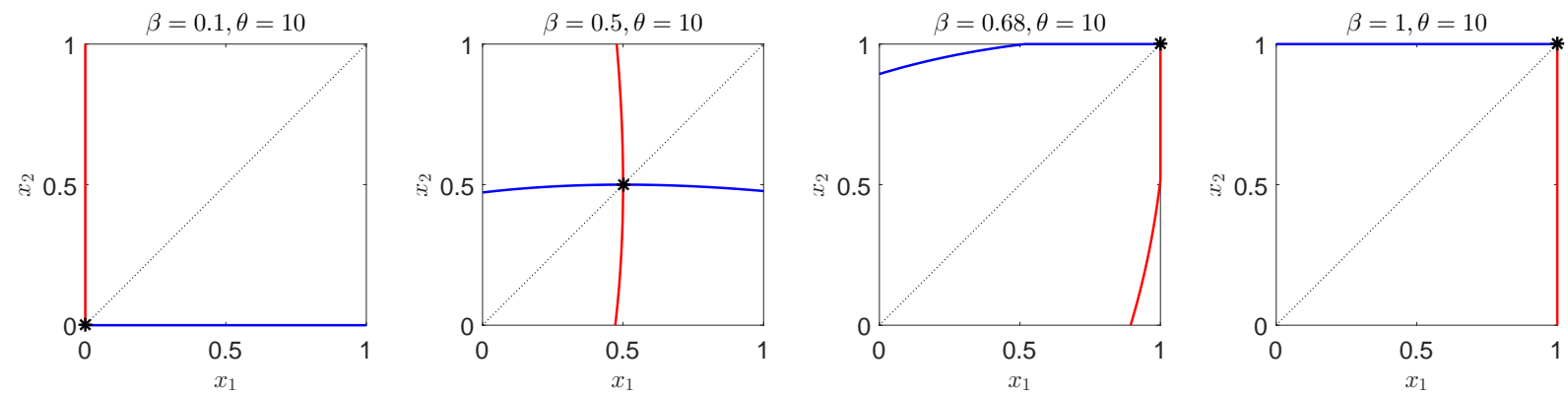

Figure 3: Combinations between the possible shapes of the best rest response functions and the resulting Nash equilibria for games $\Gamma \in \Gamma^{L N S}$.

The results of the previous Proposition are graphically sketched in Figure 3 .

Let define

$$
x_{I S}^{N E}=\frac{\beta \theta-4}{4 \beta},
$$

then we can prove the next result.

Corollary 1. For games $\Gamma \in \Gamma^{L N S}$, for any parameter configuration, there exists one and just one symmetric Nash equilibrium $\left(x^{N E}, x^{N E}\right)$ which can be either an internal or a corner equilibrium and is defined by

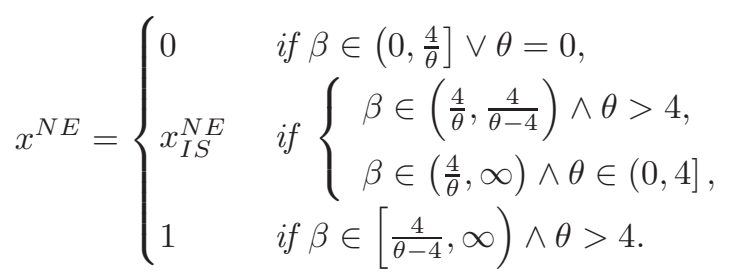




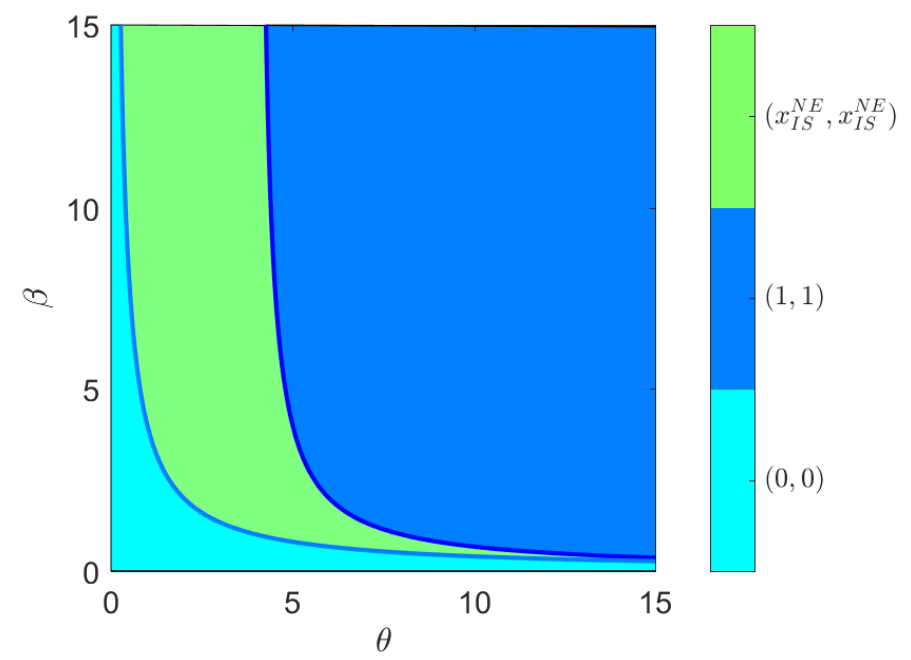

Figure 4: Possible equilibria of games $\Gamma \in \boldsymbol{\Gamma}^{L N S}$ with respect to $\theta$ and $\beta$. Darker shades of each color are used for region boundaries.

In Figure 4 we graphically represent the dependency of the contest equilibrium on $\beta$ and $\theta$.

Remark 2. The results of Propositions 1 and Q have been obtained in the particular case of linear effectiveness and cost functions. However, such results still hold in general. The related Propositions can be found in Appendix.

From 1 it is immediate to derive the following result on the effects of structural parameters on the intensity of the effort at the Nash equilibrium.

Corollary 2. For games $\Gamma \in \Gamma^{L N S}$

1. The intensity of the effort in equilibrium $x_{i}^{N E}$ is weakly increasing in the marginal productivity of the effort $\beta$ and in the agents' polarization;

2. an increase in ex ante polarization $\theta$ has the effect of reducing the interval $\left[0, \frac{4}{\theta}\right]$ of $\beta$ for which there is zero conflict, while it increases all the other intervals.

The first result of Corollary 2 is intuitive and all structural parameters affect as expected the intensity of conflict at the equilibrium. The second result is particularly interesting to interpret real contests, because it says that when there are no spillover and polarization is high, then a small increment in the marginal productivity of effort dramatically changes the equilibrium regime from no conflict to intermediate or even maximum conflict. In other words, when ex ante polarization is big, small institutional or technological changes may have a huge effect on the equilibrium behavior, which explains why the ex ante distance between counterparts' goals often is the object to monitor to minimize the risk of hugely disruptive conflicts.

\subsection{Best response functions and equilibria with spillover in outcomes}

In this section we investigate the consequences of introducing spillover in outcomes, which is obtained taking $\gamma>0$ and $\delta>0$, while keeping $\alpha=0$. We notice that, in the present case, spillover has effect on the polarization, too. The resulting payoff functions are then

$$
\pi_{i}\left(x_{i}, x_{j}\right)=-\frac{\beta x_{j}+1}{\beta x_{i}+\beta x_{j}+2}\left(\theta+\delta x_{i}+\gamma x_{j}\right)-x_{j},
$$


from which we can obtain the class of strategic form games $\Gamma^{L S O}=\left\{\Gamma=\left(\{1,2\},[0,1]^{2}, \pi\right)\right\}$ with $\pi\left(x_{i}, x_{j}\right)=$ $\pi_{1}\left(x_{1}, x_{2}\right) \times \pi_{2}\left(x_{2}, x_{1}\right)$, where $\pi_{i}$ are defined by (8).

The following results possibly get a more simple expression if we use the following two synthetic parameters,

$$
\Delta(\gamma, \delta) \equiv \gamma-\delta \text { and } \Lambda(\beta, \theta, \delta) \equiv \beta \theta-2 \delta,
$$

Let consider the interpretation of $\Delta$ and $\Lambda$. Under Assumption 4.2 we have

- $\Delta(\gamma, \delta)=\frac{\partial d_{1}}{\partial x_{2}}+\frac{\partial g_{1}}{\partial x_{1}}=-\left(\frac{\partial d_{2}}{\partial x_{1}}+\frac{\partial g_{2}}{\partial x_{2}}\right)=-\frac{\partial}{\partial x_{i}}\left[\left(g_{2}-g_{1}\right)+\left(d_{2}-d_{1}\right)\right]$, hence $\Delta$ is a measure of how both players' efforts affect the divergence between goal and defeat outcomes, i.e. a measure of the how an agent's effort affects both polarization and radicalization. In particular

$$
\gamma \uparrow \Rightarrow \Delta \uparrow \Rightarrow \gamma \uparrow \vee \delta \downarrow \text { and } \delta \downarrow \Rightarrow \Delta \uparrow \Rightarrow \gamma \uparrow \vee \delta \downarrow
$$

which means that an increment in $\Delta$ reduces the effects of $i^{\prime}$ s effort on polarization $(\delta \downarrow)$ and/or makes worse the effects of $j$ 's effort on $i$ 's defeat outcome, i.e. the effects of radicalization $(\gamma \uparrow)$. In particular

$$
\Delta \geq 0 \Leftrightarrow \gamma \geq \delta
$$

i.e. $\quad \Delta$ is positive if and only if radicalization prevails on polarization. Hence, we expect that an increment in a positive $\Delta$ would increase the equilibrium efforts.

- $\Lambda(\beta, \theta, \delta)=\theta \frac{\partial S_{i}(\alpha=0)}{\partial x_{i}}-2\left|\frac{\partial g_{i}}{\partial x_{i}}\right|$, hence $\Lambda$ is a measure of the combination between ex ante polarization and the productivity of $i$ 's effort net of the polarization effect. In particular

$$
\beta \uparrow \vee \theta \uparrow \Rightarrow \Lambda \uparrow \Rightarrow \beta \uparrow \vee \theta \uparrow \vee \delta \downarrow \text { and } \delta \downarrow \Rightarrow \Lambda \uparrow \Rightarrow \beta \uparrow \vee \theta \uparrow \vee \delta \downarrow
$$

which means that an increment in $\Lambda$ reduces the effects of $i^{\prime}$ s effort on endogenous polarization $(\delta \downarrow)$ and/or increases the marginal productivity of effort $(\beta \uparrow)$ and/or increases the ex ante polarization $(\theta \uparrow)$. In particular

$$
\Lambda(\beta, \theta, \delta) \geq 0 \Leftrightarrow \beta \theta \geq 2 \delta
$$

i.e. $\Lambda$ is positive if and only if the combination between ex ante polarization and the productivity of $i$ 's effort prevails on the polarization effect. Hence, we expect that an increment in a positive $\Lambda$ would increase the equilibrium efforts.

Now, we characterize the best response relation for this class of games.

Proposition 3. The best response functions related to games $\Gamma \in \Gamma^{L S O}$ are continuous, piecewise smooth functions given by

$$
B R_{i}\left(x_{j}\right)= \begin{cases}0 & \text { if } \beta \Delta x_{j}+\Lambda<0 \\ \min \left\{\max \left\{-x_{j}-\frac{2}{\beta}+\frac{1}{\beta} \sqrt{\left(\beta \Delta x_{j}+\Lambda\right)\left(\beta x_{j}+1\right)}, 0\right\}, 1\right\} & \text { if } \beta \Delta x_{j}+\Lambda \geq 0 .\end{cases}
$$

Note that with the radicalization assumption, the best response function can be either constantly equal to 0 , constantly equal to 1, strictly non-increasing, strictly non-decreasing or hump-shaped.

Notice that the continuity of the best response function guarantees the existence of the Nash equilibrium. The main difference with the result of Proposition 1, despite the possibility of having strictly non-increasing best response functions, lies in its behavior at fixed points $\bar{x}$. In the case of no spillover, best response functions must be flat at $\bar{x}$, which guarantees the equilibrium uniqueness. Such property no more holds when 

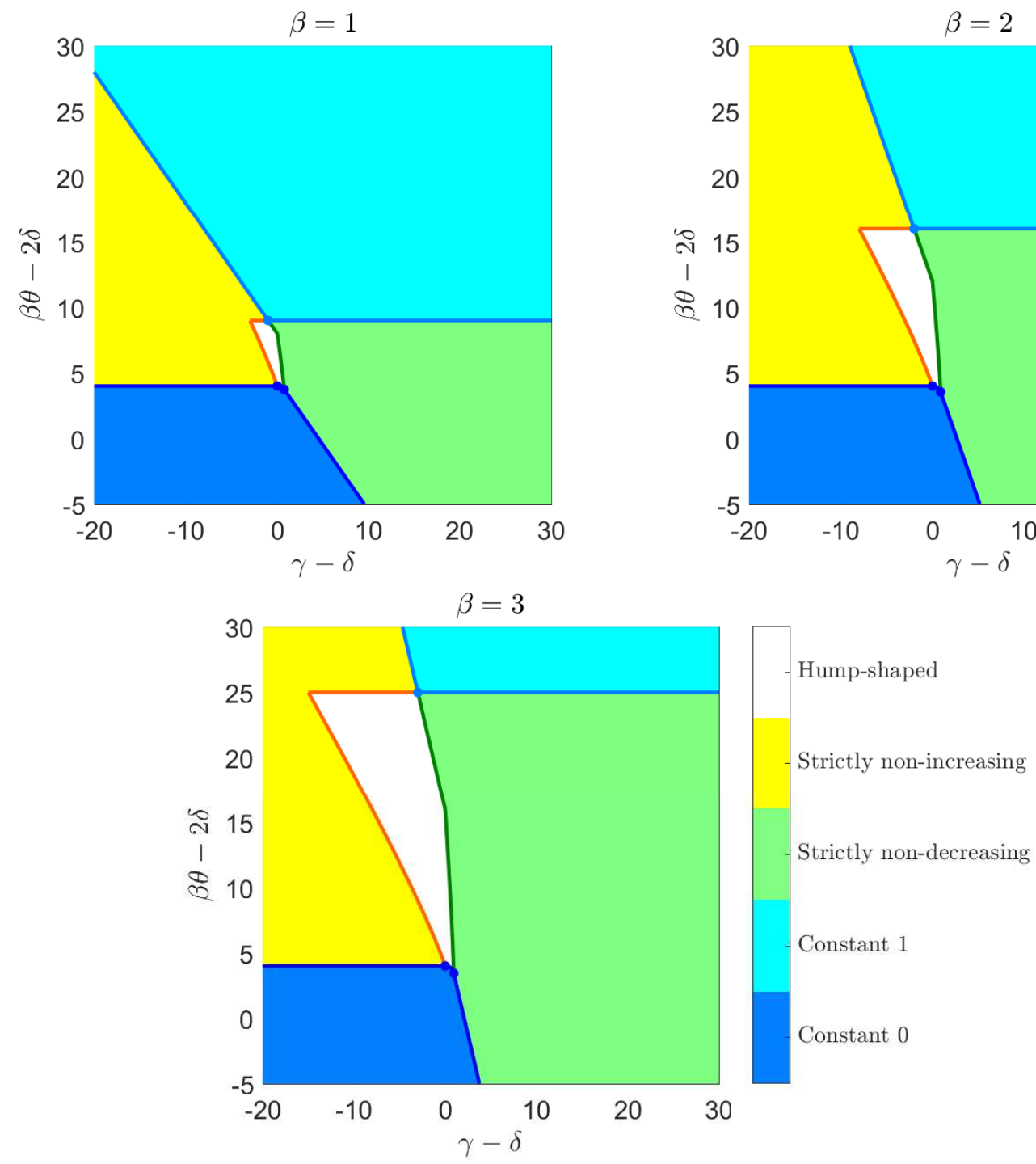

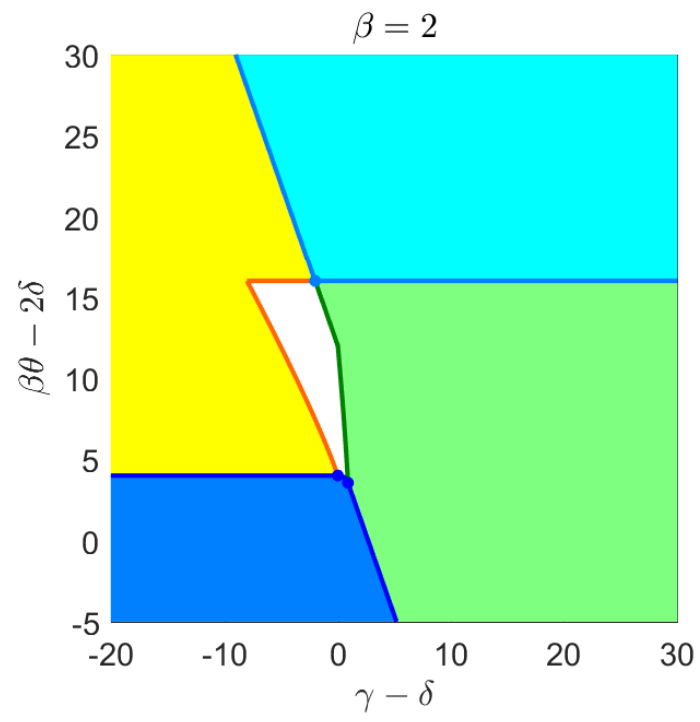

Figure 5: Possible best response functions of games $\Gamma \in \Gamma^{L S O}$ with respect to $\beta \theta-2 \delta$ and $\gamma-\delta$. Blue (respectively cyan) region represents parameters' combinations for which the best response is constantly equal to 1 (respectively to 0 ). Green (respectively yellow) region represents parameters' combinations for which the best response function is strictly non-decreasing (respectively strictly non-decreasing). Darker shades of each color are used for region boundaries and corners.

there are spillover in polarization and in outcomes. This is the root of the next Proposition. However, first let consider the specific case $\Delta=\Lambda=4$ : then it is immediate to see that best response function (9) reduces to $B R_{i}\left(x_{j}\right)=x_{j}$. This means that in this very peculiar case any strategy $x_{i} \in[0,1]$ is a Nash equilibrium. We avoid to further deal with this case, so in the remainder of this section we assume that at least one of the two synthetic parameters $\Delta=\gamma-\delta$ and $\Lambda=\beta \theta-2 \delta$ is different from 4 .

Proposition 4. When the best response function for a game $\Gamma \in \Gamma^{L S O}$ is

- strictly non-decreasing, then we can have

- a unique symmetric corner Nash equilibrium; 
- three symmetric Nash equilibria, which correspond to an internal equilibrium and the two corner equilibria. For some parameter configurations, the internal equilibrium can coincide with one of the corner equilibria;

- hump-shaped, then we can have

- one symmetric internal Nash equilibrium;

- one symmetric internal Nash equilibrium, together with two asymmetric internal Nash equilibria;

- one symmetric internal Nash equilibrium together with two asymmetric boundary Nash equilibria;

- strictly non-decreasing, then we can have

- one symmetric internal Nash equilibrium;

- one symmetric internal Nash equilibrium together with two asymmetric boundary Nash equilibria;

- one symmetric internal Nash equilibrium together with the two corner asymmetric Nash equilibria $(1,0)$ and $(0,1)$

- constant, then the unique Nash equilibrium is a symmetric corner equilibrium.

In Figure 6 we represent the various scenarios resulting from Proposition 4 avoiding to report the trivial case of constant best response functions.

Let us introduce

$$
x_{I S}^{N E}=-\left(\frac{1}{\beta}\right)\left[\frac{\Lambda-4}{\Delta-4}\right]=\frac{(\beta \theta-2 \delta)-4}{\beta[4-(\gamma-\delta)]},
$$

and

$$
\begin{aligned}
x_{1, A S}^{N E} & =-\left(\frac{1}{\beta}\right)\left[\frac{\Delta(\sqrt{\Delta+4}+\sqrt{\Delta})-\Lambda(\sqrt{\Delta+4}-\sqrt{\Delta})}{2 \Delta \sqrt{\Delta}}\right]= \\
& =\frac{[(\beta \theta-2 \delta)-(\gamma-\delta)] \sqrt{\frac{(\gamma-\delta)+4}{(\gamma-\delta)}}-[(\beta \theta-2 \delta)+(\gamma-\delta)]}{2 \beta(\gamma-\delta)} \\
x_{2, A S}^{N E} & =-\left(\frac{1}{\beta}\right)\left[\frac{\Delta(\sqrt{\Delta+4}+\sqrt{\Delta})+\Lambda(\sqrt{\Delta+4}-\sqrt{\Delta})}{2 \Delta \sqrt{\Delta}}\right]= \\
& =-\frac{\left[(\beta \theta-2 \delta)-\left(\gamma-\delta \sqrt{\frac{(\gamma-\delta)+4}{(\gamma-\delta)}}\right)\right]-[(\beta \theta-2 \delta)+(\gamma-\delta)]}{2 \beta(\gamma-\delta)}
\end{aligned}
$$

and

$$
\begin{aligned}
& x_{0, B}^{N E}=-\left(\frac{1}{\beta}\right)(2-\sqrt{\Lambda})=\frac{\sqrt{\beta \theta-2 \delta}-2}{\beta}, \\
& x_{1, B}^{N E}=-\left(\frac{1}{\beta}\right)(2+\beta-\sqrt{(\beta+1)(\beta \Delta+\Lambda)})=\frac{\sqrt{(\beta+1)[(\beta \theta-2 \delta)+\beta(\gamma-\delta)]}}{\beta} .
\end{aligned}
$$

We can then summarize the set of possible Nash equilibria in the following Corollary.

Corollary 3. For any game $\Gamma \in \Gamma^{L S O}$ and for any parameter configuration, there exist up to three Nash equilibria, which can be all symmetric or one symmetric and two asymmetric. In particular,

1. when the internal symmetric equilibrium exists it is given by $\left(x_{I S}^{N E}, x_{I S}^{N E}\right)$, 

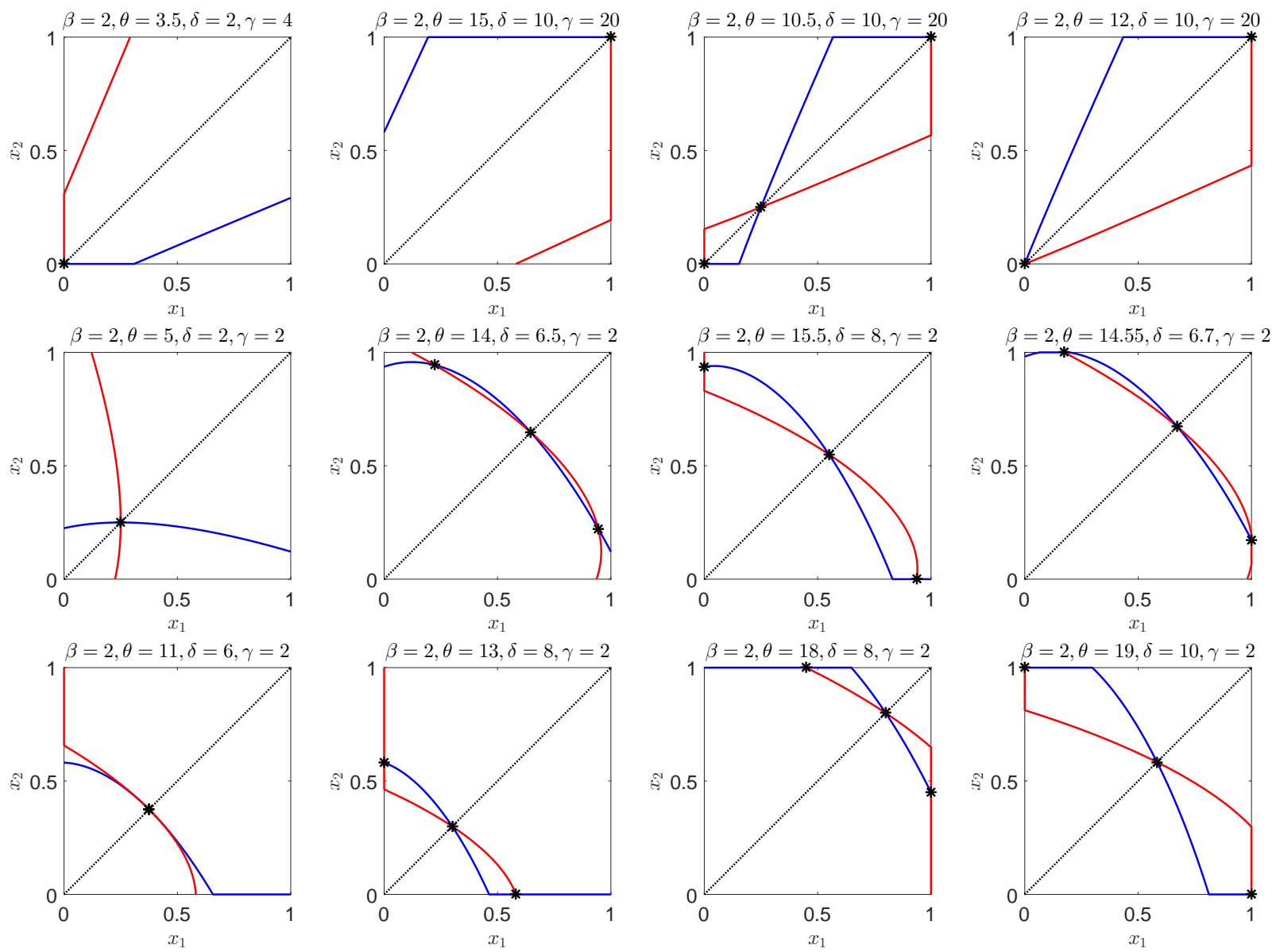

Figure 6: Combinations between the possible shapes of the best response functions and the resulting Nash equilibria for games $\Gamma \in \Gamma^{L S O}$. In the first row we report the cases corresponding to strictly non-decreasing functions, in the second row those corresponding to hump-shaped functions, in the third row those corresponding to strictly non-increasing functions.

2. when the internal asymmetric equilibria exist they are given by $\left(x_{1, A S}^{N E}, x_{2, A S}^{N E}\right)$ and $\left(x_{2, A S}^{N E}, x_{1, A S}^{N E}\right)$

3. when the boundary equilibria exist they are given by either $\left(x_{0, B}^{N E}, 0\right),\left(0, x_{0, B}^{N E}\right)$ or $\left(x_{1, B}^{N E}, 1\right),\left(1, x_{1, B}^{N E}\right)$

Corner equilibria can be either symmetric or asymmetric.

Hence, we might conclude with the following corollary.

Corollary 4. For any game $\Gamma \in \Gamma^{L S O}$ there exist

- multiple symmetric Nash equilibria if and only if

$$
\left\{\begin{array}{l}
\beta(4-\Delta)+4 \leq \Lambda \leq 4 \\
\Delta \geq 4
\end{array}\right.
$$

- asymmetric Nash equilibria if and only if

$$
\left\{\begin{array}{l}
4<\Lambda<\beta(4-\Delta)+4 \\
\Delta<-4
\end{array}\right.
$$


- a unique symmetric equilibrium if and only if

$$
\left\{\begin{array} { l } 
{ \Lambda \leq 4 , } \\
{ \Delta < \frac { 4 ( \beta + 1 ) - \Lambda } { \beta } , }
\end{array} \cup \left\{\begin{array} { l } 
{ \Lambda > 4 , } \\
{ \Delta \geq \frac { 4 ( \beta + 1 ) - \Lambda } { \beta } , }
\end{array} \cup \left\{\begin{array}{l}
\Delta \geq 4 \\
\Lambda>4 \\
\Delta<\frac{4(\beta+1)-\Lambda}{\beta}
\end{array}\right.\right.\right.
$$

We remark that if condition (13) is fulfilled with a strict inequality, then we have three distinct equilibria, while when the equality occurs the internal equilibrium coincides with either $(0,0)$ and $(1,1)$, and we actually have the two symmetric corner equilibria. In what follows we focus on the case of three distinct equilibria.

We notice that multiplicity of symmetric equilibria, i.e. condition (13), necessarily requires $\Delta>4$, as well as multiplicity of asymmetric equilibria, i.e. condition (14), necessarily requires $\Delta<-4$. This is in agreement with the case of no spillover, in which $\Delta=0$ and no multiplicity of equilibria is possible. Moreover, if the divergence between goal and defeat outcomes is suitably small $(|\Delta|<4$, ) the possible arising scenarios are the same of the case with no spillover, and only a unique symmetric equilibrium is possible. This means that the measure of how both players' efforts affect the divergence between goal and defeat outcomes should be big enough or small enough to get multiple equilibria, while for intermediate values we get uniqueness as if there are no outcome spillover. Similarly, multiplicity of symmetric equilibria, i.e. condition (13), necessarily requires $\beta(4-\Delta)+4<\Lambda<4$, as well as multiplicity of asymmetric equilibria, i.e. condition (14), necessarily requires $4<\Lambda<\beta(4-\Delta)+4$. This means that the measure of the combination between ex ante polarization and the productivity of $i$ 's effort net of the polarization effect should be upper intermediate to get multiple asymmetric equilibria, while for low intermediate values we might get multiple symmetric equilibria. More generally, if endogenous radicalization is sufficiently large with respect to endogenous polarization, multiple symmetric equilibria can arise, for intermediate values of $\Lambda$. On the other hand, if endogenous radicalization is sufficiently small with respect to endogenous polarization, multiple asymmetric equilibria can arise, again for intermediate values of $\Lambda$. Since $\Delta$ is a measure of how both players' efforts affect the divergence between goal and defeat outcomes, i.e. a measure of the how an agent's effort affects both polarization and radicalization these means that the existence of multiple symmetric equilibria requires a huge endogenous effect of effort on radicalization net of polarization, while the existence of multiple asymmetric equilibria requires a huge endogenous effect of effort on polarization net of radicalization.

Let now consider all our four parameters, i.e. productivity of effort $\beta$, endogenous radicalization $\gamma$, endogenous polarization $\delta$ and ex ante polarization $\theta$. The fact that in our model there are four parameters means that it is impossible to have a full picture of the relative behavior of these parameters. However its is interesting to consider the necessary and sufficient conditions w.r.t $\beta$ and $\theta$, for given $\gamma$ and $\delta$, and, vice versa, the necessary and sufficient conditions w.r.t $\delta$ and $\gamma$, for given $\beta$ and $\theta$.

Let consider $\delta$ and $\gamma$, for given $\beta$ and $\theta$. The necessary and sufficient conditions for multiple symmetric equilibria can be written as

$$
\left\{\begin{array}{l}
\beta(4-\Delta)+4<\Lambda<4 \\
\Delta>4
\end{array} \Leftrightarrow \frac{\beta \theta-4}{2}<\delta<\min \left\{\gamma-4 ; \frac{\beta(\gamma+\theta)-4(\beta+1)}{\beta+2}\right\}\right.
$$

which means that endogenous polarization should be bounded below by the interaction between productivity of effort and ex ante polarization and above by a measure of endogenous radicalization. This means that, as the following picture shows, both endogenous polarization and endogenous radicalization should be big enough, even if endogenous polarization can't grow to quickly.

On the other hand, the necessary and sufficient conditions for multiple asymmetric equilibria can be 


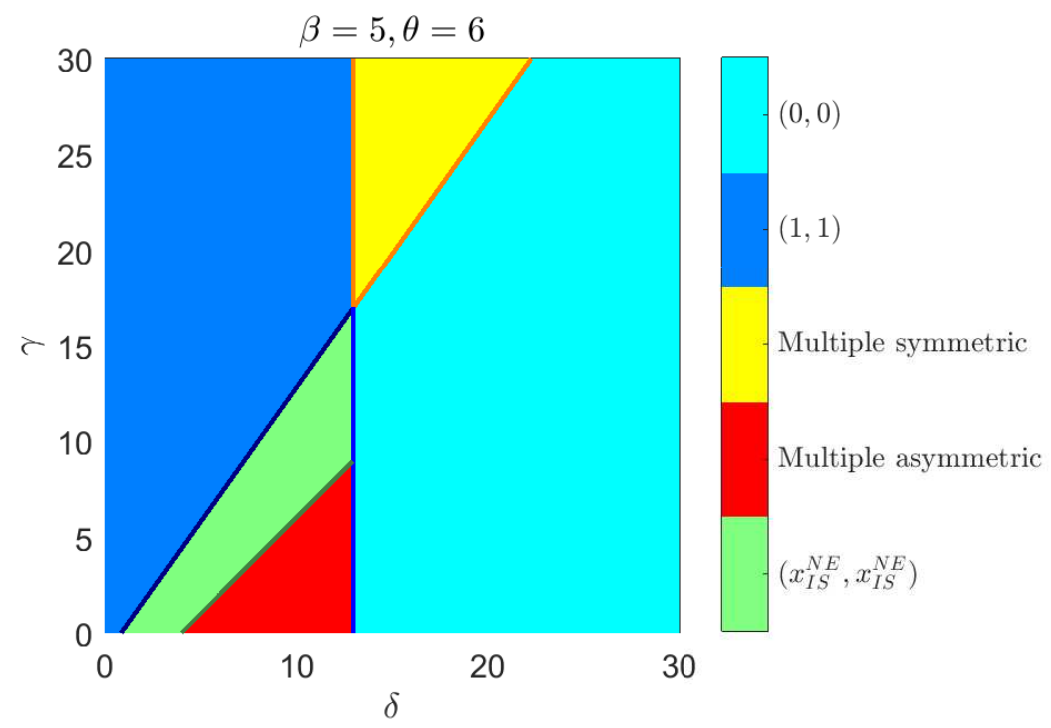

Figure 7: $(\delta, \gamma)$ regions where there are multiple symmetric, asymmetric or unique NE. Boundaries are represented by darker colors with respect to the region to which they belong.

written as

$$
\left\{\begin{array}{l}
4<\Lambda<\beta(4-\Delta)+4 \\
\Delta<-4
\end{array} \Leftrightarrow \max \left\{\gamma+4 ; \frac{\beta(\gamma+\theta)-4(\beta+1)}{\beta+2}\right\}<\delta<\frac{\beta \theta-4}{2}\right.
$$

which means that endogenous polarization should be bounded above by the interaction between productivity of effort and ex ante polarization and below by a measure of endogenous radicalization. This means that, as the following picture shows, both endogenous polarization and endogenous radicalization should be significant but they are restricted in their values, while a crucial role is played by the interaction between productivity of effort and ex ante polarization that should be big enough.

It is also interesting to consider the necessary and sufficient conditions w.r.t $\beta$ and $\theta$, for given $\delta$ and $\gamma$.

The necessary and sufficient conditions for multiple symmetric equilibria can be written as

$$
\left\{\begin{array} { l } 
{ \beta ( 4 - \Delta ) + 4 < \Lambda < 4 } \\
{ \Delta > 4 }
\end{array} \Leftrightarrow \left\{\begin{array}{l}
\frac{4+2 \delta}{\theta+(\Delta-4)}<\beta<\frac{4+2 \delta}{\theta} \\
\Delta>4
\end{array}\right.\right.
$$

which means that the productivity of effort should be bounded below and above by two values that are decreasing in ex ante polarization but increasing in endogenous polarization. On the other hand, the necessary and sufficient conditions for multiple asymmetric equilibria can be written as

$$
\left\{\begin{array} { l } 
{ 4 < \Lambda < \beta ( 4 - \Delta ) + 4 } \\
{ \Delta < - 4 }
\end{array} \Leftrightarrow \left\{\begin{array} { l } 
{ \frac { 4 + 2 \delta } { \theta } < \beta < \frac { 4 + 2 \delta } { \theta + ( \Delta - 4 ) } } \\
{ \Delta < - 4 \wedge \theta > 4 - \Delta }
\end{array} \vee \left\{\begin{array}{l}
\frac{4+2 \delta}{\theta}<\beta \\
\Delta<-4 \wedge \theta<\Delta-4
\end{array}\right.\right.\right.
$$

which means that the range of possible values for the productivity of effort depends on the value of ex ante polarization. These cases are sketched in Figure 8 in which we also report how thresholds vary depending on $\delta$ and $\gamma$, when the dependence is uniform with respect to the other parameters. These figures shows that the regions for different et of equilibria in the $(\theta, \beta)$ space have a similar behavior w.r.t. the case of no spillover, where for big value of ex ante polarization, a small increase in the productivity of effort is sufficient to shift from a region with no effort to an intermediate situation, possibly with multiple symmetric or asymmetric equilibria, to a region with maximum effort only. 

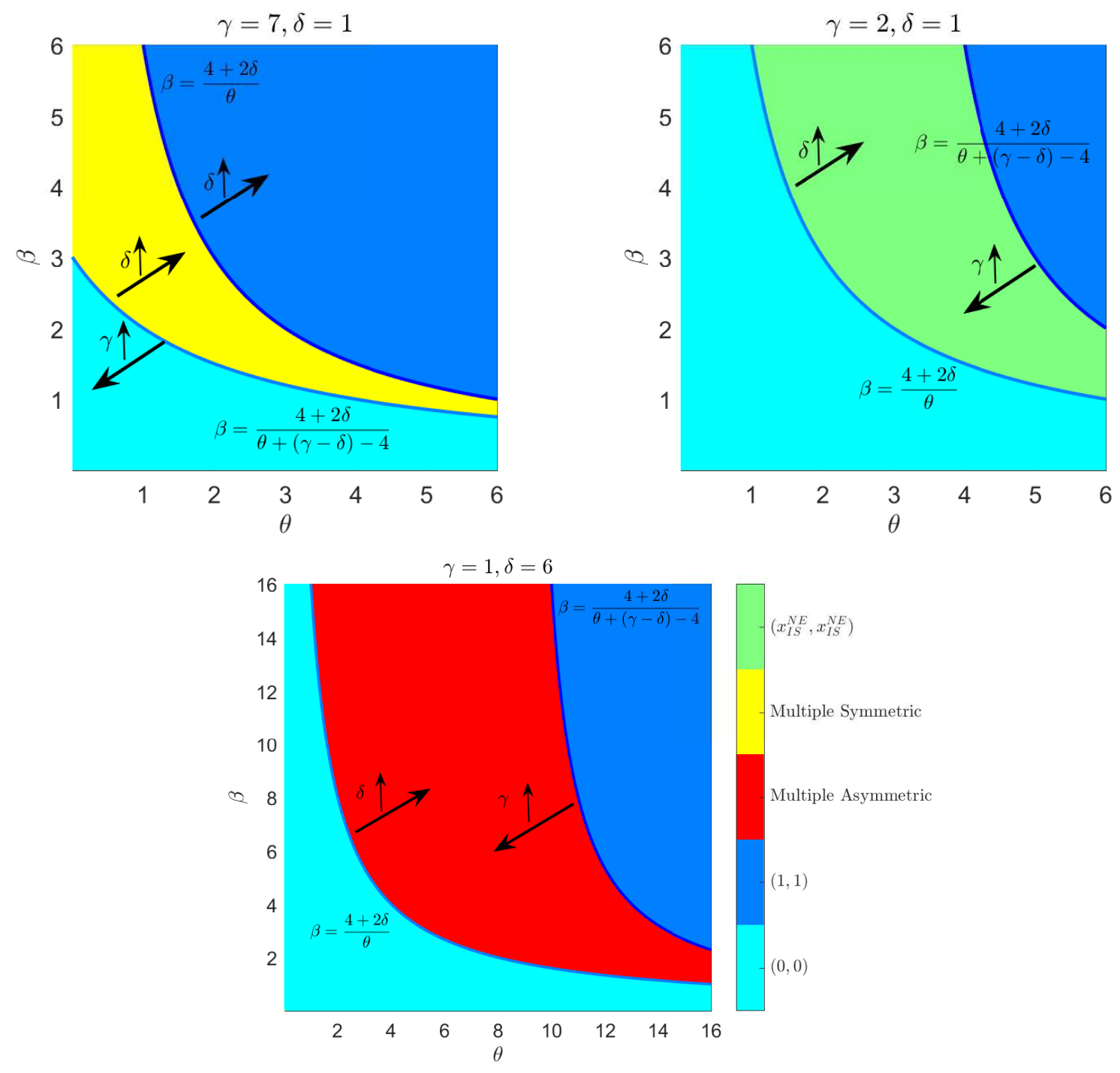

Figure 8: $(\theta, \beta)$ regions where there are multiple symmetric, asymmetric or unique NE. Boundaries are represented by darker colors with respect to the region to which they belong.

Finally, the correspondence between parameters $\beta \theta-2 \delta, \gamma-\delta$ and $\beta$ and the possible sets of Nash equilibria are reported in Figure 9, for some fixed values of productivity of effort.

In the following Corollaries we collect several results concerning the effects of the parameters on the equilibria, also focusing on their role on the occurrence of multiple and asymmetric equilibria. We start studying how the intensity of effort at the various Nash equilibria varies depending on $\beta, \delta, \gamma$ and $\theta$. We assume that the parameters' perturbation is such that it does not affect the existence of the particular equilibrium. In this sense, the results of Corollaries 516 and 7 are local. In the first Corollary we focus on the internal symmetric equilibrium.

Corollary 5. 1. Let $\beta \theta-2 \delta>4$ (or equivalently $\gamma-\delta<4$ ). Then there is a unique equilibrium and an increase of $\beta, \gamma$ or $\theta$ leads to an increase of $x_{I S}^{N E}$, while an increase of $\delta$ leads to a decrease of $x_{I S}^{N E}$.

2. Conversely, let $\beta \theta-2 \delta<4$ (or equivalently $\gamma-\delta>4$ ). Then there are multiple symmetric equilibria and an increase of $\beta, \gamma$ or $\theta$ leads to a decrease of $x_{I S}^{N E}$, while an increase of $\delta$ leads to an increase of 

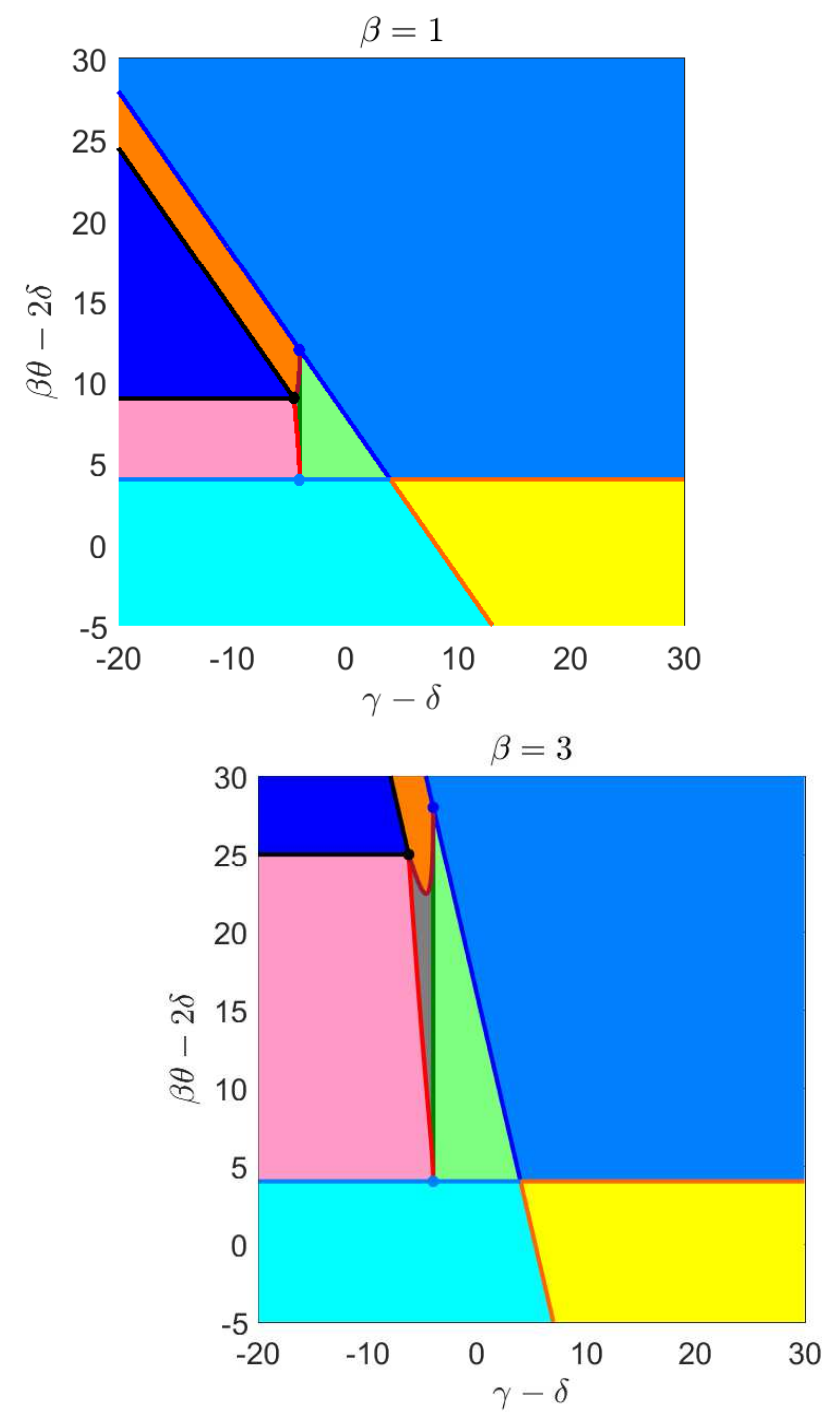
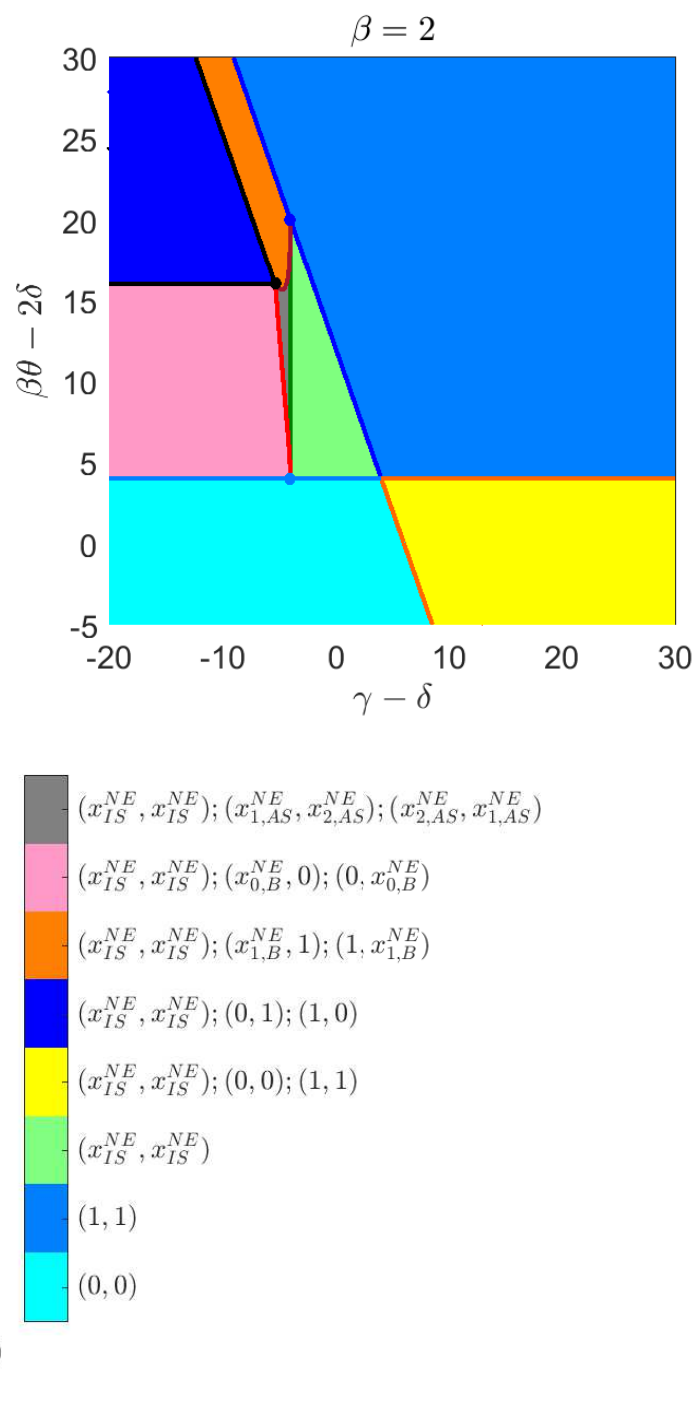

Figure 9: Possible equilibria of games $\Gamma \in \Gamma^{L S O}$ with respect to $\gamma-\delta$ and $\beta \theta-2 \delta$, for various values of $\beta$. Darker shades of each color are used for region boundaries and corners.

$$
x_{I S}^{N E} .
$$

Now consider the internal asymmetric equilibrium. We remark that, from the definitions of $x_{1, A S}^{N E}$ and $x_{2, A S}^{N E}$, we have that internal asymmetric equilibria are $\left(x_{1, A S}^{N E}, x_{2, A S}^{N E}\right)$ and $\left(x_{2, A S}^{N E}, x_{1, A S}^{N E}\right)$ where $x_{1, A S}^{N E}>x_{2, A S}^{N E}$ provided that $\gamma-\delta<-4$.

Corollary 6. Increasing $\beta, \gamma$ or $\theta$ leads to an increase of $x_{1, A S}^{N E}$, while increasing $\delta$ leads to a decrease of $x_{1, A S}^{N E}$.

Increasing $\beta$ or $\theta$ leads to an increase of $x_{2, A S}^{N E}$. If $\beta$ is sufficiently small, then increasing $\gamma$ or decreasing $\delta$ decreases $x_{2, A S}^{N E}$. If $\beta$ is sufficiently large and $\beta \theta-2 \delta$ is sufficiently small, increasing $\gamma$ or decreasing $\delta$ decreases $x_{2, A S}^{N E}$, while if $\beta$ is sufficiently large and $\beta \theta-2 \delta$ is sufficiently large, increasing $\gamma$ or decreasing $\delta$ increases $x_{2, A S}^{N E}$.

Finally, the case of asymmetric equilibria on the boundaries.

Corollary 7. The intensity of effort $x_{0, B}^{N E}$ increases with $\beta$ and $\theta$, while it decreases as $\delta$ increases. The intensity of effort $x_{1, B}^{N E}$ increases with $\beta, \theta$ and $\gamma$, while it decreases as $\delta$ increases. 
These results on local comparative statics require some comments, considering the different possible scenarios:

1. when there is a unique equilibrium, then the equilibrium effort in equilibrium is increasing in the marginal productivity of effort, in endogenous radicalization and in the ex ante polarization but, may be surprisingly, it is decreasing in the endogenous polarization. The point is that with a bigger $\delta$, an increment in effort has two effects: on one hand it increase the likelihood of winning the contest, on the other hand it pushes further away the two players goals, while an increase in $\gamma$ makes a default worse, incentivizing more effort;

2. the results are more complex, however qualitatively similar when there are multiple asymmetric equilibria

3. when there are multiple symmetric equilibria, surprisingly the effects of the parameters are completely reversed: the equilibrium effort in equilibrium is decreasing in the marginal productivity of effort, in endogenous radicalization and in the ex ante polarization but it is increasing in the endogenous polarization. However it should be emphasized that in this case, an increase in endogenous polarization increases the regions with zero effort equilibria while an increase in endogenous radicalization increase the regions of maximum effort equilibria.

We believe these results shows the importance of distinguishing endogenous polarization and radicalization, because of the different role played on equilibrium effort.

\subsection{Best response functions and Nash equilibria with spillover in effectivity function}

In this section we investigate the consequences of introducing spillover in CST, in particular on effectivity functions. In this case we assume $\alpha>0, \gamma=\delta=0$, i.e. a positive direct destructiveness effect.

Then, the resulting payoff functions are

$$
\pi_{i}\left(x_{i}, x_{j}\right)=-a \frac{\beta x_{j}\left(1-\alpha x_{i}\right)+1}{\beta x_{i}\left(1-\alpha x_{j}\right)+\beta x_{j}\left(1-\alpha x_{i}\right)+2} \theta-x_{i}+b,
$$

from which we obtain the class of strategic form game $\Gamma^{L S E}=\left\{\Gamma=\left(\{1,2\},[0,1]^{2}, \pi\right)\right\}$ with $\pi\left(x_{i}, x_{j}\right)=$ $\pi_{1}\left(x_{1}, x_{2}\right) \times \pi_{2}\left(x_{2}, x_{1}\right)$, where $\pi_{i}$ are defined by (16)

Let us introduce

$$
\tilde{x}_{j}=\frac{4 \alpha-\beta+\beta \theta-4+\sqrt{(4 \alpha+\beta)\left(4 \alpha+\beta-4 \theta-2 \beta \theta+\beta \theta^{2}\right)}}{2 \beta(1-2 \alpha+\alpha \theta} .
$$

Firstly we characterize the best response functions for this class of games.

Proposition 5. Let us consider games $\Gamma \in \Gamma^{L S E}$. Then

- if $\alpha<1 / 2$, the best response function of player $i$ is the continuous, piecewise smooth function

$$
B R_{i}\left(x_{j}\right)=\max \left\{\min \left\{\frac{\beta-\sqrt{\beta(4 \alpha+\beta-\alpha \beta \theta)}}{2 \alpha \beta}, 1\right\}, 0\right\}
$$

for which if $B R_{i}\left(x_{j}\right)=x_{j}$, then $B R_{i}^{\prime}\left(x_{j}\right)=0$

- if $\alpha \geq 1 / 2$ and $\beta \theta<4+\beta / \alpha$, the best response function of player $i$ is the continuous, piecewise smooth function

$$
B R_{i}\left(x_{j}\right)= \begin{cases}\max \left\{\min \left\{\frac{\beta-\sqrt{\beta(4 \alpha+\beta-\alpha \beta \theta)}}{2 \alpha \beta}, 1\right\}, 0\right\} & \text { if } 0 \leq x_{j}<\frac{1}{2 \alpha} \\ 0 & \text { if } \frac{1}{2 \alpha} \leq x_{j} \leq 1\end{cases}
$$


for which if $B R_{i}\left(x_{j}\right)=x_{j}$, then $B R_{i}^{\prime}\left(x_{j}\right)=0$

- If $\alpha \geq 1 / 2$ and $\beta \theta=4+\beta / \alpha$, the best response correspondence of player $i$ is the upper-hemicontinuous correspondence

$$
B R_{i}\left(x_{j}\right)= \begin{cases}\max \left\{\min \left\{\frac{\beta-\sqrt{\beta(4 \alpha+\beta-\alpha \beta \theta)}}{2 \alpha \beta}, 1\right\}, 0\right\} & \text { if } 0 \leq x_{j}<\frac{1}{2 \alpha}, \\ {[0,1]} & \text { if } x_{j}=\frac{1}{2 \alpha}, \\ 0 & \text { if } \frac{1}{2 \alpha}<x_{j} \leq 1,\end{cases}
$$

whose restriction to $\left\{x_{j} \neq 1 / 2 \alpha\right\}$ is a continuous function;

- if $\alpha \geq 1 / 2$ and $\beta \theta>4+\beta / \alpha$, the best response correspondence of player $i$ is the upper-hemicontinuous correspondence

$$
B R_{i}\left(x_{j}\right)= \begin{cases}\max \left\{\min \left\{\frac{\beta-\sqrt{\beta(4 \alpha+\beta-\alpha \beta \theta)}}{2 \alpha \beta}, 1\right\}, 0\right\} & \text { if } 0 \leq x_{j}<\frac{1}{2 \alpha}, \\ 1 & \text { if } \frac{1}{2 \alpha} \leq x_{j}<\tilde{x}_{j}, \\ \{0,1\} & \text { if } x_{j}=\tilde{x}_{j}, \\ 0 & \text { if } \tilde{x}_{j}<x_{j} \leq 1,\end{cases}
$$

whose restriction to $\left\{x_{j} \neq \tilde{x}_{j}\right\}$ is a continuous function.

Depending on $\alpha, \beta$ and $\theta$, if $\tilde{x}_{j} \notin[0,1]$ then the best response function can be either constantly equal to 0 , constantly equal to 1 , strictly non-decreasing or hump-shaped. Conversely, if $\tilde{x}_{j} \in[0,1]$, the best response correspondence is non-decreasing for $x_{j}<\tilde{x}_{j}$ and constantly equal to 0 for $x_{j}>\tilde{x}_{j}$.

In Figure 10 we report the resulting shapes of the best response depending on parameters $\alpha$ and $\theta$ for different values of $\beta$, distinguishing in particular when it is a function or a correspondence.

The main difference between the case with spillover on effectivity function and those without spillover or spillover in the outcome is that in the present case the best response can be a correspondence and not a function. In particular, we can have that the best response correspondence is not convex-valued, hence the existence of the Nash equilibrium is not guaranteed anymore. The situation is studied in the following Proposition.

Proposition 6. When the best response for games $\Gamma \in \Gamma^{L S E}$ is a function and is

- strictly non-decreasing, then the unique Nash equilibrium is a corner equilibrium;

- hump-shaped, then the unique Nash equilibrium is internal and symmetric;

- constant, then the unique Nash equilibrium is a symmetric corner equilibrium;

When the best response for games $\Gamma \in \Gamma^{L S E}$ is not a function, then we can either have a symmetric internal equilibrium (which can eventually become a corner equilibrium) or no equilibria.

For any parameter configuration, no asymmetric equilibria occur.

In Figure 11 we report the various scenarios resulting from the previous Proposition, neglecting the trivial case of constant best response functions. The previous results can be summarized in the following Corollary, in which we use

$$
x_{I S}^{N E}=\frac{1}{2 \alpha}-\frac{\sqrt{\beta(4 \alpha+\beta-\alpha \beta \theta)}}{2 \alpha \beta} .
$$

Corollary 8. For game $\Gamma^{L S E}$, we can have either a unique symmetric equilibrium or no equilibrium. 

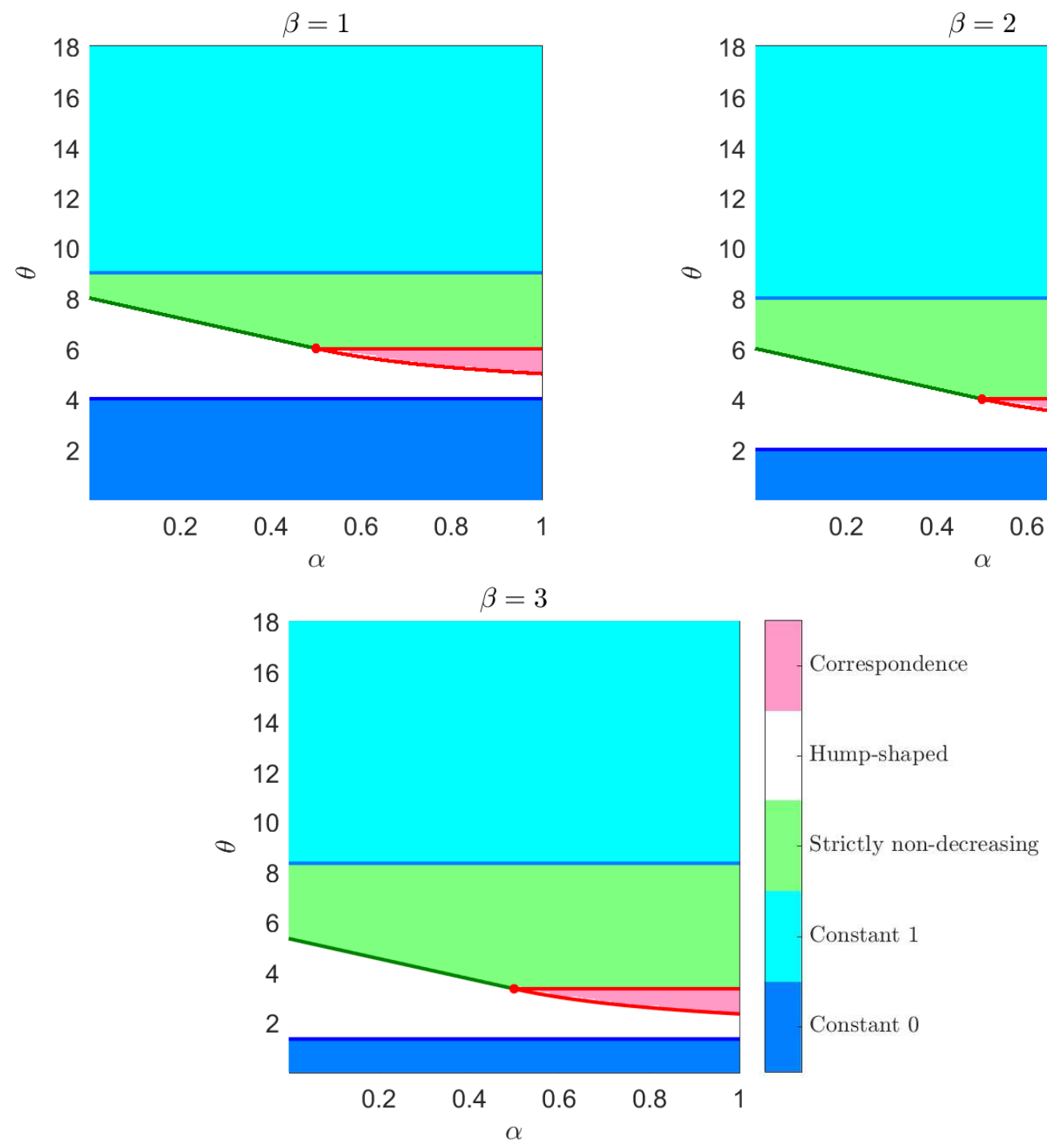

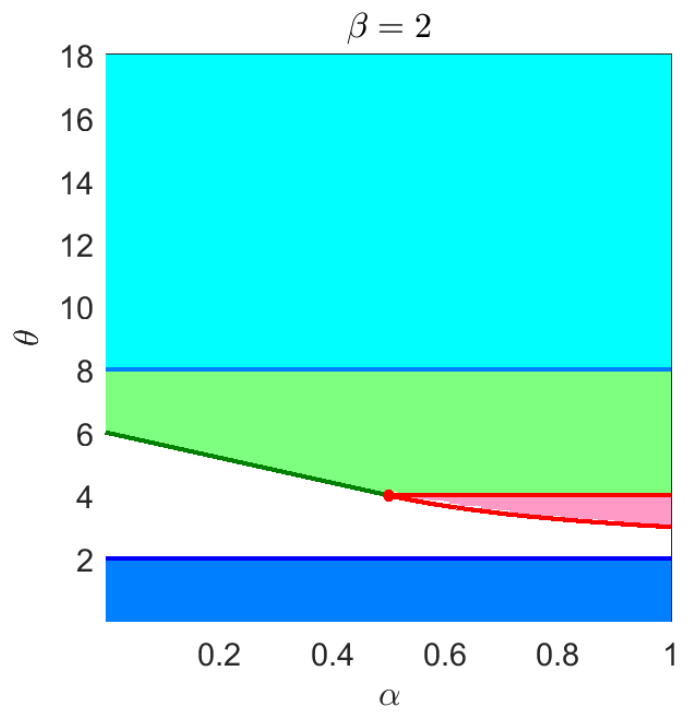

Figure 10: Possible shapes of best response functions/correspondences for games $\Gamma \in \Gamma^{L S E}$ with respect to $\theta$ and $\alpha$, for different values of $\beta$. Blue (respectively cyan) region represents parameters' combinations for which the best response is constantly equal to 1 (respectively to 0 ). Green (respectively white) region represents parameters' combinations for which the best response function is strictly non-decreasing (respectively humpshaped). Pink region represents parameters' combinations for which the best response is a correspondence. Darker shades of each color are used for region boundaries and corners.

As for the case with spillover on outcomes, we investigate the effects of the parameters on the equilibrium, also focusing on its existence or not. Also in this case the proof is omitted.

Corollary 9. The intensity of effort $x_{I S}^{N E}$ increases as $\beta, \theta$ and $\alpha$ increase.

We have no equilibria if and only if

$$
\left\{\begin{array}{l}
\alpha \geq 1 / 2, \\
\frac{4}{\beta} \leq \theta \leq \frac{4 \alpha+\beta}{\alpha \beta} .
\end{array}\right.
$$

Let note that we have the usual effects of the marginal productivity of effort and of ex ante polarization on the equilibrium effort as well as of the direct destructiveness parameter, however when these effects are 

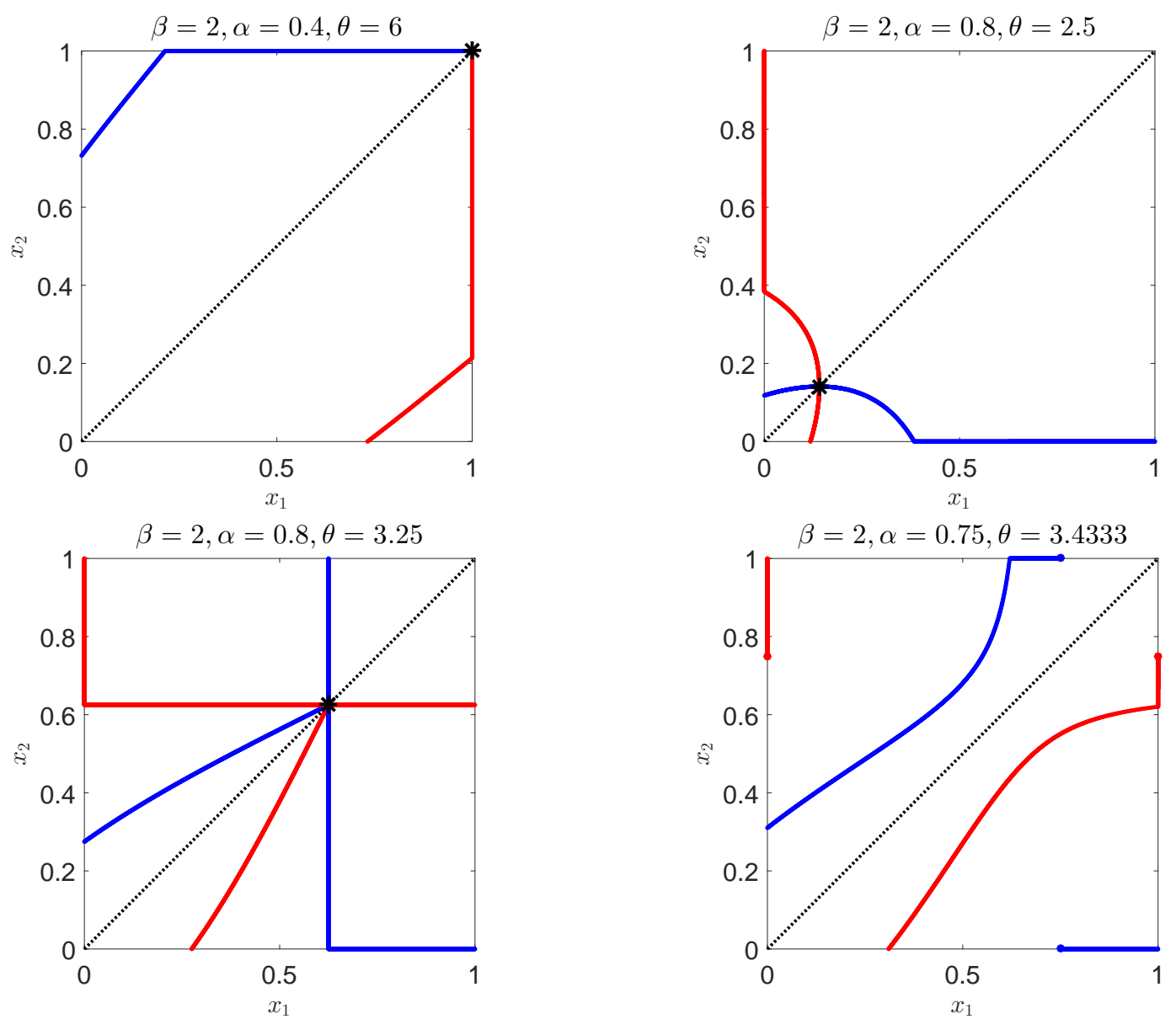

Figure 11: Combinations between the possible shapes of the best response functions/correspondences and the resulting Nash equilibria for a game $\Gamma \in \Gamma^{L S E}$. In the first row we report the cases in which the best response is a function, in the second row we report the cases in which the best response is only a correspondence.

big enough, then they destroy (pure strategy) equilibrium existence because of the discontinuity in the best reply functions.

\section{Conclusion}

To the best of our knowledge, the results of this paper are quite innovative, because no contest model is able to generate all these results, connecting the characteristics of the set of equilibria to fundamental micro properties of the contest model. In particular Chowdhury and Sheremeta 2011a construct a generalized Tullock contest and characterize the unique symmetric equilibrium, while Szidarovszky and Okuguchi 1997 analyze existence and uniqueness for general but standard models, however we think that our basic parameters have more neat and useful interpretations making applications more immediate, as we have shown in previous corollaries. Moreover Chowdhury and Sheremeta 2011b use their generalized contest model to derive the conditions that imply the existence of multiple asymmetric equilibria, however in our model we find not only multiple asymmetric equilibria, but also multiple symmetric equilibria that can easily be Pareto ordered. Again, the conditions implying these kind of equilibria have a neat strategic interpretation. In particular our results provides clues to interpret real contests' situations stressing the role of contest success technology $(\beta)$ and of polarization $(\theta)$, of endogenous polarization and radicalization $(\gamma, \delta)$ and the consequences of 

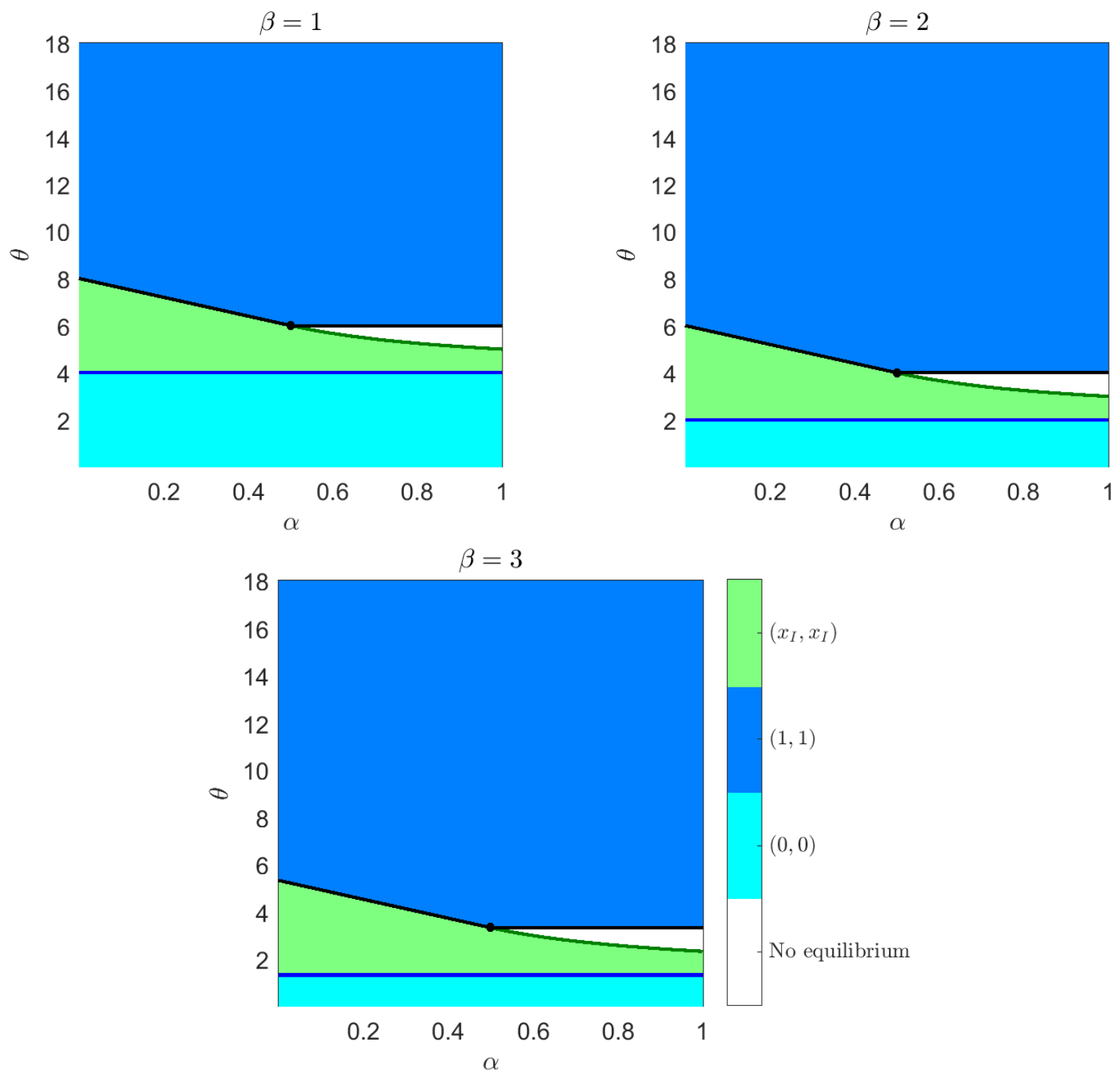

Figure 12: Possible equilibria of games $\Gamma \in \Gamma^{L S E}$ with respect to $\alpha$ and $\theta$, for various values of $\beta$. Darker shades of each color are used for region boundaries and corners.

destructive possibilities $(\alpha)$ for the properties of the set of Nash equilibria, to induce uniqueness or multiplicity, symmetry and asymmetry.

Our future work will focus on out of equilibrium dynamics, which is analyzed in the companion paper Cavalli et al. 2016, on the ways of modelling players' multiple goals and multiple contest tools with different effects, e.g. defensive versus offensive efforts, which would mean different effectivity and cost functions.

\section{References}

[1] Baik Kyung Hwan 1993. Effort levels in contests. Economic Letters 41, 363-367

[2] Corchon Luis C. 2007. The theory of contests: a survey. Review of Economic Design, 11, 2: 69-100.

[3] Cornes Richard and Roger Hatley 2912. Risk aversion in symmetric and asymmetric contests. Economic Theory, 51: 247-275. 
[4] Chowdhury Subhasish M. and Roman M. Sheremeta 2011a. A generalized Tullock contest. Public Choice, 147: 413-420.

[5] Chowdhury Subhasish M. and Roman M. Sheremeta 2011b. Multiple equilibria in Tullock contests. Economic Letters, 112: 216-219.

[6] Dasgupta, Ani and Kofi O. Nti 1998. Designing an optimal contest. European Journal of Political Economy 14: 587-603

[7] Garfinkel, Michelle R. and Stergios Skaperdas 2007. Economics of Conflict: An Overview. Chapter 22 in Handbook of Defense Economics, vol. II, Sandler and Hartley eds., North Holland, Amsterdam.

[8] Konrad Kai A. 2009. Strategy and Dynamics in Contests. Oxford University Press, Oxford.

[9] Krueger, Anne O. 1974. The Political Economy of the Rent-Seeking Society. America Economic Review, VOL. 64, N. 3: 291-303.

[10] Hausken Kjell 2005. Production and Conflict Models Versus Rent-Seeking Models. Public Choice, 123: $59-93$

[11] Hirshleifer Jack 1989. Conflict and Rent-Seeking Success Functions: Ratio vs. Difference Models of Relative Success. Public Choice, 63, 101-112.

[12] Hirshleifer Jack 1991. The Paradox of Power. Economics and Politics, 3, 177-200.

[13] Hirshleifer Jack 1995a. Anarchy and its Breakdown. Journal of Political Economy, 103, 27-52.

[14] Hirshleifer Jack 1995b. Theorizing about conflict, In Handbook of Defence Economics, Vol. I Hartley and Sandler, North Holland, Amsterdam.

[15] Hirshleifer Jack and Alfred E. Osborne 2001. Truth, Effort, and the Legal Battle. Public Choice.

[16] Neary Hugh M. 1997. A comparison of rent-seeking models and economic models of conflict. Public Choice 93: 373-388.

[17] Nitzan Shmuel 1994. Modelling rent-seeking contests. European Journal of Political Economy, 10: 41-60.

[18] Pérez-Castrillo J. David and T. Verdier 1992. A general analysis of rent-seeking games. Public Choice 73: 335-350.

[19] Persson Torsten and Guido Tabellini 2000. Political Economics, MIT Press, Cambridge, Mass.

[20] Riaz Khalid, Jason H. Shogren and Stanley R. Johnson 1995. A general model of rent seeking for public goods. Public Choice, 82, 243-259.

[21] Szidarovszky Ferenc and Koji Okuguchi 1997. On the Existence and Uniqueness of Pure Nash Equilibrium in Rent-Seeking games. Games and Economic Behavior, 18: 135-140.

[22] Tullock, Gordon 1967. The Welfare Costs of Tariffs, Monopolies, and Theft, Western Economic Journal, 5, 3: 224-232.

[23] Tullock Gordon 1980. Efficient Rent Seeking, In Towards a Theory of the Rent-Seeking Society Buchanan, Tollison and Tullock, Texas A\&M University Press, Texas. 


\section{Appendix}

This Section collects the proofs of the Propositions and Corollaries of Sections 3.1, 3.2 and 3.3 respectively concerning the family of games $\Gamma^{N S}, \Gamma^{L S O}$ and $\Gamma^{L S E}$.

We remark that, thanks the symmetry of the considered games, we have that $B R_{i}(z)=B R_{j}(z)$ for any $z \in[0,1]$, so in what follows we can focus on just the best response function $B R_{i}\left(x_{j}\right)$ of player $i$ with respect to the strategy $x_{j}$ of player $j$.

\subsection{Proofs of section 3.1}

We start noticing that, from (4), we have

$$
\begin{gathered}
\partial_{x_{i}} \pi_{i}\left(x_{i}, x_{j}\right)=\frac{\beta\left(\theta+\beta \theta x_{j}\right)}{\left(\beta x_{i}+\beta x_{j}+2\right)^{2}}-1, \quad \partial_{x_{i}}^{2} \pi_{i}\left(x_{i}, x_{j}\right)=-\frac{2 \beta^{2}\left(\theta+\beta \theta x_{j}\right)}{\left(\beta x_{i}+\beta x_{j}+2\right)^{3}}, \\
\partial_{x_{i} x_{j}}^{2} \pi_{i}\left(x_{i}, x_{j}\right)=\frac{\beta^{3} \theta\left(x_{i}-x_{j}\right)}{\left(\beta x_{i}+\beta x_{j}+2\right)^{3}} .
\end{gathered}
$$

Since $\partial_{x_{i}}^{2} \pi_{i}\left(x_{i}, x_{j}\right)<0$, the payoff function is strictly concave for any parameters' configuration.

Let us introduce function $x_{+}:[-1 / \beta,+\infty) \rightarrow \mathbb{R}$ defined by

$$
x_{+}(z)=-z-\frac{2}{\beta}+\sqrt{\left(z+\frac{1}{\beta}\right) \theta},
$$

which will be used for the characterization of the best response function. The properties of $x_{+}$are investigated in the following Lemma, whose proof is omitted as straightforward.

Lemma 1. Function $x_{+}$is a strictly concave function for which

$$
x_{+}\left(z_{m}\right)=z_{m} \Leftrightarrow z_{m}=\frac{\beta \theta-4}{4 \beta},
$$

where $z_{m}$ is the unique maximum point of $x_{+}$. It is strictly increasing (respectively decreasing) in $\left[-1 / \beta, z_{m}\right.$ ) (respectively in $\left(z_{m},+\infty\right)$ ), in which $x_{+}(z)>z$ (respectively $\left.x_{+}(z)<z\right)$.

In the next Lemma we compute the best response function.

Lemma 2. The best response function $B R_{i}:[0,1] \rightarrow[0,1]$ is given by

$$
B R_{i}\left(x_{j}\right)=\min \left\{\max \left\{x_{+}\left(x_{j}\right), 0\right\}, 1\right\}
$$

where $x_{+}$is defined by (24).

Proof. We start solving $x_{i}=\operatorname{argmax}_{z \in[0,1]} \pi_{i}\left(z, x_{j}\right)$ for a fixed $x_{j} \in[0,1]$. From (23) , we have that $\partial_{x_{i}} \pi_{i}\left(x_{i}, x_{j}\right) \geq$ 0 leads to

$$
-\beta^{2} x_{i}^{2}+\left(-2 x_{j} \beta^{2}-4 \beta\right) x_{i}-\left(\beta^{2} x_{j}^{2}-\theta \beta^{2} x_{j}+4 \beta x_{j}-\theta \beta+4\right) \geq 0 .
$$

Assuming that $x_{j}>-1 / \beta$, inequality (27) is solved $x_{-}\left(x_{j}\right) \leq x_{i} \leq x_{+}\left(x_{j}\right)$, where

$$
x_{ \pm}\left(x_{j}\right)=-x_{j}-\frac{2}{\beta} \pm \sqrt{\left(x_{j}+\frac{1}{\beta}\right) \theta},
$$

are real values for any positive $\beta$ and $\theta$. Noticing that $x_{-}\left(x_{j}\right)<0$, for a fixed $x_{j}$, we have three cases

- $x_{+}\left(x_{j}\right) \leq 0$ : the marginal payoff is negative for any $x_{i} \in[0,1]$, so the payoff function $\pi_{i}\left(x_{i}, x_{j}\right)$ is strictly decreasing any $x_{i} \in[0,1]$ and attains its maximum for $x_{i}=0$. In this case we have $B R_{i}\left(x_{j}\right)=0$. 
- $x_{+}\left(x_{j}\right) \in(0,1)$ : the marginal payoff is positive for any $x_{i} \in\left[0, x_{+}\left(x_{j}\right)\right)$ and negative for any $x_{i} \in$ $\left(x_{+}\left(x_{j}\right), 1\right]$, so the payoff function $\pi_{i}\left(x_{i}, x_{j}\right)$ is strictly increasing for $x_{i} \in\left[0, x_{+}\left(x_{j}\right)\right)$ and strictly decreasing for $x_{i} \in\left(x_{+}\left(x_{j}\right), 1\right]$, and attains its maximum for $x_{i}=x_{+}\left(x_{j}\right)$. Then, we have $B R_{i}\left(x_{j}\right)=$ $x_{+}\left(x_{j}\right)$.

- $x_{+}\left(x_{j}\right) \geq 1$ : the marginal payoff is positive for any $x_{i} \in[0,1]$, so the payoff function $\pi_{i}\left(x_{i}, x_{j}\right)$ is strictly increasing for any $x_{i} \in[0,1]$ and attains its maximum for $x_{i}=1$. We then have $B R_{i}\left(x_{j}\right)=1$.

This allows concluding.

In the next two Lemmas we prove two properties of the best response function.

Lemma 3. If $B R_{i}\left(\tilde{x}_{j}\right)=0$ for some $\tilde{x}_{j} \in[0,1]$, then $B R_{i}\left(x_{j}\right)=0$ for any $x_{j}>\tilde{x}_{j}$. If $B R_{i}\left(\tilde{x}_{j}\right)=1$ for some $\tilde{x}_{j} \in[0,1]$, then $B R_{i}\left(x_{j}\right)=1$ for any $x_{j}>\tilde{x}_{j}$.

Proof. If $B R_{i}\left(\tilde{x}_{j}\right)=0$, then $x_{+}\left(\tilde{x}_{j}\right) \leq 0<\tilde{x}_{j}$ and, from Lemma 1, we have $x_{+}^{\prime}\left(\tilde{x}_{j}\right)<0$. Since $x_{+}$is concave, it decreases for any $x_{j}>\tilde{x}_{j}$, and then, again from Lemma 1 we have $x_{+}\left(\tilde{x}_{j}\right)<0$, i.e. $B R_{i}\left(x_{j}\right)=0$.

For the second part, if $B R_{i}\left(\tilde{x}_{j}\right)=1$ for some $\tilde{x}_{j}<1$, we have that $B R_{i}\left(\tilde{x}_{j}\right)=x_{+}\left(\tilde{x}_{j}\right)>\tilde{x}_{j}$. From Lemma 11. this means that $x_{+}$is increasing for any $x_{j} \in\left(\tilde{x}_{j}, z_{m}\right)$. Since $z_{m}=x_{+}\left(z_{m}\right)>x_{+}\left(\tilde{x}_{j}\right)=1$, this means that $x_{+}$is increasing, and hence larger than 1 , for any $x_{j}>\tilde{x}_{j}$. This concludes the proof.

Lemma 4. If $B R_{i}\left(x_{j}\right)=x_{j}$ for some $x_{j} \in[0,1]$, then $B R_{i}^{\prime}\left(x_{j}\right)=0$.

Proof. If $B R_{i}(0)=0$, from Lemma 3 we have that the best response is null for any $x_{j} \in[0,1]$, so we trivially have $B R_{i}^{\prime}(0)=0$.

If $B R_{i}(1)=1$, from Lemma 2 we have that $x_{+}(1) \geq 1$. If $x_{+}(1)>1$, since $x_{+}$is a continuous function, there exists a suitable interval $\left(x_{j}, 1\right)$ such that $x_{+}\left(x_{j}\right)>1$, and in which hence $B R_{i}\left(x_{j}\right)=1$. In this case we trivially have $B R_{i}^{\prime}(1)=0$. Conversely, if $x_{+}(1)=1$, from Lemma 2 we have that 1 is the maximum point of $x_{+}$and so $B R_{i} \equiv x_{+}$is a suitable left neighborhood $(1-\epsilon, 1]$ of 1 , which provides $B R_{i}^{\prime}(1)=x_{+}^{\prime}(1)=0$.

If $B R_{i}\left(x_{j}\right)=x_{j}$ for some $x_{j} \in(0,1)$, we can conclude by noticing that $B R_{i} \equiv x_{+}$in a suitable neighborhood $\left(x_{j}-\epsilon, x_{j}+\epsilon\right)$ of $x_{j}$, which provides $B R_{i}^{\prime}\left(x_{j}\right)=x_{+}^{\prime}\left(x_{j}\right)=0$.

The previous Lemmas allow proving Propositions and Corollaries of Section 3.1 .

Proof of Propositions 1 and [2. From Lemmas 1 and 2 we have that $B R_{i}$ is a piecewise smooth and continuous function, while from Lemma 4 we have that $B R_{i}^{\prime}\left(x_{j}\right)$ vanishes if $B R_{i}\left(x_{j}\right)=x_{j}$.

The classification of best responses of games $\Gamma \in \Gamma^{L N S}$ then follows from the possible behaviors of $z_{m}$, considering the properties of $x_{+}$shown in Lemma 1 and the expression of $B R_{i}$ provided by Lemma 2

If $z_{m} \leq 0$, then we have that $x_{+}\left(x_{j}\right) \leq 0=B R_{i}\left(x_{j}\right)$ for any $x_{j} \in[0,1]$. The unique possible Nash equilibrium is indeed $(0,0)$.

We notice that if $z_{m}>0$, we have $x_{+}(0)>0$, as otherwise, by intermediate value theorem, equation $x_{+}(z)=z$ would have two positive solutions, which is not possible from Lemma 1 ,

Then, if $0<z_{m}<1$, there exists $b \leq 1$ (with $b=1$ if and only if $x_{+}(1)>0$ ) such that $B R_{i}\left(x_{j}\right)=$ $x_{+}\left(x_{j}\right)$ for $x_{j} \in[0, b]$ and $B R_{i}\left(x_{j}\right)=0$ for $x_{j} \in(b, 1]$. The best response function is then increasing for $x_{j} \in\left[0, z_{m}\right)$, decreasing for $x_{j} \in\left(z_{m}, b\right]$ and null for $x_{j} \in(b, 1]$ (where this last interval can be empty). Since $B R_{i}\left(z_{m}\right)=z_{m}$, we have that $\left(z_{m}, z_{m}\right)$ is indeed a Nash equilibrium. Moreover, from (25) we can not have further solutions of $B R_{i}(x)=x$, and is the unique symmetric Nash equilibrium.

If $z_{m} \geq 1$ and $B R_{i}(0)<1$, there exists $b \leq 1 \leq z_{m}$ (with $\beta=1$ if and only if $z_{m}=1$ ) such that $B R_{i}\left(x_{j}\right)=x_{+}\left(x_{j}\right)$ for $x_{j} \in[0, b]$ and $B R_{i}\left(x_{j}\right)=1$ for $x_{j} \in(b, 1]$. The best response function is then increasing for $x_{j} \in[0, b)$, and constant for $x_{j} \in(b, 1]$ (where this last interval can be empty). 
Finally, if $z_{m} \geq 1$ and $B R_{i}(0) \geq 1$, since $x_{+}$is increasing up to $z_{m}>1$, we have $B R_{i}\left(x_{j}\right)=1$. The unique possible Nash equilibrium is indeed $(0,0)$.

We stress that, since the previous conditions are mutually excluding, they are also necessary conditions for the occurrence of each scenario.

Due to the shape of $x_{+}$, we can not have asymmetric equilibria, since the graphs of the best response functions $y=B R_{i}(x)$ and $x=B R_{i}(y)$ are subsets of regions which have in common only the set of symmetric points $\left\{P \in[0,1]^{2}: P=(x, x), x \in[0,1]\right\}$. In fact, recalling also the previous considerations, we have that if the best response function is strictly non-decreasing, the graph of $y=B R_{i}(x)$ lies above the bisector of plane $(x, y)$, while the graph of $x=B R_{i}(y)$ lies below the bisector of plane $(x, y)$. Similarly, in the case of hump-shaped best response functions, we have that

- the graph of $y=B R_{i}(x)$ lies above the bisector of plane $(x, y)$ for $x \in\left[0, z_{m}\right]$, while the graph of $x=B R_{i}(y)$ lies below the bisector of plane $(x, y)$ for $y \in\left[0, z_{m}\right]$

- the graph of $y=B R_{i}(x)$ lies below the bisector of plane $(x, y)$ for $x \in\left[z_{m}, 1\right]$, while the graph of $x=B R_{i}(y)$ lies above the bisector of plane $(x, y)$ for $y \in\left[z_{m}, 1\right]$.

In the next two propositions we provide the analytic description of the regions reported in Figure 2 and 4. namely the characterization of best responses of games $\Gamma \in \Gamma^{L N S}$ and the occurrence of either internal or corner equilibria with respect to $\beta$ and $\theta$

Proposition 7. We have that the best response functions of games $\Gamma \in \Gamma^{L N S}$ are

a) constantly equal to 0 if and only if $\beta \theta \leq 4$, which corresponds to the blue region of Figure 国:

b) hump-shaped if and only $4<\beta \theta<4 \beta+4$, which corresponds to the white region of Figure 0 ;

c) strictly non-decreasing if and only $4 \beta+4 \leq \beta \theta<(2+\beta)^{2}$, which corresponds to the green region of Figure 圆:

d) constantly equal to 1 if and only if $\beta \theta \geq(2+\beta)^{2}$, which corresponds to the cyan region of Figure .

Proof. The previous regions are obtained by simply solving $z_{m} \leq 0$ (when $B R_{i} \equiv 0$ ), $0<z_{m}<1$ (for hump-shaped best response functions), $z_{m} \geq 1$ with $x_{+}(0)<1$ (for the strictly non-decreasing case) and $x_{+}(0) \geq 1\left(\right.$ when $\left.B R_{i} \equiv 1\right)$.

Proposition 8. We have

a) the corner equilibrium $(0,0)$ if and only if $\beta \theta \leq 4$, which corresponds to the cyan region of Figure 国;

b) the internal equilibrium if and only $4<\beta \theta<4 \beta+4$, which corresponds to the green region of Figure 国:

c) the corner equilibrium $(1,1)$ if and only if $\beta \theta \geq 4 \beta+4$, which corresponds to the blue region of Figure 4.

Proof. Case a) is obtained from $z_{m} \leq 0$; case b) from $0<z_{m}<1$; case c) from $z_{m} \geq 1$. 
Proof of Corollaries 1 and , We start rewriting conditions provided by Proposition 8 in terms of $\beta$. If $\theta>4$ we have

$$
x_{I S}^{N E}= \begin{cases}0 & \text { if } \beta \leq \frac{4}{\theta}, \\ \in(0,1) & \text { if } \frac{4}{\theta}<\beta<\frac{4}{\theta-4}, \\ 1 & \text { if } \beta \geq \frac{4}{\theta-4} .\end{cases}
$$

while if $\theta \leq 4$ we have

$$
x_{I S}^{N E}= \begin{cases}0 & \text { if } \beta \leq \frac{4}{\theta}, \\ \in(0,1) & \text { if } \beta>\frac{4}{\theta} .\end{cases}
$$

We obtained (7). This means that, increasing $\beta$ from 0 to $+\infty$, the Nash equilibrium is initially the corner equilibrium $(0,0)$, it subsequently becomes the internal equilibrium $\left(x_{I S}^{N E}, x_{I S}^{N E}\right)$ and then it (possibly) becomes the corner equilibrium $(1,1)$. Notice that $x_{I S}^{N E}$ is increasing in $\beta$.

Rewriting conditions provided by Proposition 8 in terms of $\theta$

$$
x_{I S}^{N E}= \begin{cases}0 & \text { if } \theta \geq \frac{4}{\beta}, \\ \in(0,1) & \text { if } \frac{4}{\beta}<\theta<\frac{4(\beta+1)}{\beta}, \\ 1 & \text { if } \theta \leq \frac{4(\beta+1)}{\beta} .\end{cases}
$$

we have that $\theta$ from 0 to $+\infty$ the Nash equilibrium is initially the corner equilibrium $(0,0)$, it subsequently becomes the internal equilibrium $\left(x_{I S}^{N E}, x_{I S}^{N E}\right)$ and then it (possibly) becomes the corner equilibrium $(1,1)$. Notice that $x_{I S}^{N E}$ is increasing in $\theta$. The last result is straightforward and concludes the proof.

The next Proposition is devoted to the generalization of the previous results.

Proposition 9. Let us assume that there is no spillover in the agents' effectiveness of the efforts, the contest polarization is constant with respect to the efforts and there is no spillover in the cost function. Then, the following scenarios are the only that can occur:

\begin{tabular}{|c|l|c|}
\hline & Best replies & Nash equilibrium \\
\hline (I) & $\begin{array}{l}\text { BR }\left(x_{j}\right) \text { is a positive function } \\
\text { with unimodal shape in }[0,1]\end{array}$ & $\left(x_{i}^{N E}, x_{j}^{N E}\right) \in(0,1) \times(0,1)$ \\
\hline (II) & $\begin{array}{l}B R_{i}\left(x_{j}\right) \text { is a positive function } \\
\text { with unimodal shape in }\left[0, x_{a}\right] \text { and } \\
\text { null in }\left[x_{a}, 1\right]\end{array}$ & $\left(x_{i}^{N E}, x_{j}^{N E}\right) \in(0,1) \times(0,1)$ \\
\hline (III) & $\begin{array}{l}B R_{i}\left(x_{j}\right) \text { is a positive function in- } \\
\text { creasing in }\left[0, x_{a}\right] \text { and equal to } 1 \text { in } \\
{\left[x_{a}, 1\right]}\end{array}$ & $\left(x_{i}^{N E}, x_{j}^{N E}\right)=(1,1)$ \\
\hline (IV) & $B R_{i}\left(x_{j}\right)$ is constantly null & $\left(x_{i}^{N E}, x_{j}^{N E}\right)=(0,0)$ \\
\hline (V) & $B R_{i}\left(x_{j}\right)$ is constantly equal to 1 & $\left(x_{i}^{N E}, x_{j}^{N E}\right)=(1,1)$ \\
\hline
\end{tabular}

where $x_{a} \in(0,1]$ In all the situations we have that $B R^{\prime}\left(x^{N E}\right)=0$ and that the equilibrium is unique.

Proof. Thanks to the symmetry, we can focus only on player 1. Under the previous assumptions, we have that effort and cost functions are actually one dimensional, and, for the sake of notation, we will again denote with $S$ and $C$. The payoff function becomes

$$
\pi_{1}\left(x_{1}, x_{2}\right)=-\frac{S\left(x_{2}\right)}{S\left(x_{1}\right)+S\left(x_{2}\right)} \theta-C\left(x_{1}\right)
$$


with marginal payoff

$$
\partial_{x_{1}} \pi_{1}\left(x_{1}, x_{2}\right)=\frac{S\left(x_{2}\right) S^{\prime}\left(x_{1}\right)}{\left(S\left(x_{1}\right)+S\left(x_{2}\right)\right)^{2}} \theta-C^{\prime}\left(x_{1}\right)
$$

and

$$
\begin{aligned}
& \partial_{x_{1}}^{2} \pi_{1}\left(x_{1}, x_{2}\right)=\frac{S\left(x_{2}\right)\left(S\left(x_{1}\right)+S\left(x_{2}\right)\right) \partial_{x_{1}}^{2} S\left(x_{1}\right)-2 S\left(x_{2}\right)\left(\partial_{x_{1}} S\left(x_{1}\right)\right)^{2}}{\left(S\left(x_{1}\right)+S\left(x_{2}\right)\right)^{3}} \theta-C^{\prime \prime}\left(x_{1}\right) \\
& \partial_{x_{1} x_{2}}^{2} \pi_{1}\left(x_{1}, x_{2}\right)=\frac{\left(S\left(x_{1}\right)+S\left(x_{2}\right)\right)^{2} \partial_{x_{2}} S\left(x_{2}\right) \partial_{x_{1}} S\left(x_{1}\right)-2\left(S\left(x_{1}\right)+S\left(x_{2}\right)\right) S\left(x_{2}\right) \partial_{x_{1}} S\left(x_{1}\right) \partial_{x_{2}} S\left(x_{2}\right)}{\left(S\left(x_{1}\right)+S\left(x_{2}\right)\right)^{4}} \theta \\
&=\frac{\left(S\left(x_{1}\right)+S\left(x_{2}\right)\right) \partial_{x_{2}} S\left(x_{2}\right) \partial_{x_{1}} S\left(x_{1}\right)-2 S\left(x_{2}\right) \partial_{x_{1}} S\left(x_{1}\right) \partial_{x_{2}} S\left(x_{2}\right)}{\left(S\left(x_{1}\right)+S\left(x_{2}\right)\right)^{3}} \theta \\
&=\left(S\left(x_{1}\right)-S\left(x_{2}\right)\right) \frac{\partial_{x_{2}} S\left(x_{2}\right) \partial_{x_{1}} S\left(x_{1}\right)}{\left(S\left(x_{1}\right)+S\left(x_{2}\right)\right)^{3}} \theta
\end{aligned}
$$

From now on, we fix $x_{2}$ and we study the properties of $\pi_{1}\left(x_{1}, x_{2}\right), \partial_{x_{1}} \pi_{1}\left(x_{1}, x_{2}\right), \partial_{x_{1}}^{2} \pi_{1}\left(x_{1}, x_{2}\right)$ and $\partial_{x_{1} x_{2}}^{2} \pi_{1}\left(x_{1}, x_{2}\right)$ as functions of $x_{1}$ only.

Firstly, we notice that $\partial_{x_{1}}^{2} \pi_{1}\left(x_{1}, x_{2}\right)<0$, so $\partial_{x_{1}} \pi_{1}\left(x_{1}, x_{2}\right)$ is strictly decreasing, which means that $\pi_{1}\left(x_{1}, x_{2}\right)$ is strictly concave. Regarding the monotonicity of $\pi_{1}$, since $\partial_{x_{1}} \pi_{1}\left(x_{1}, x_{2}\right)$ is strictly decreasing, we can have that

1. $\forall x_{1} \in[0,1) \partial_{x_{1}} \pi_{1}\left(x_{1}, x_{2}\right)>0$, so $\pi_{1}\left(x_{1}, x_{2}\right)$ is strictly increasing

2. there exists $x_{M} \in(0,1)$ such that $\partial_{x_{1}} \pi_{1}\left(x_{1}, x_{2}\right)>0$ for $x_{1} \in\left[0, x_{M}\right)$ and $\partial_{x_{1}} \pi_{1}\left(x_{1}, x_{2}\right)<0$ for $x_{1} \in\left(x_{M}, 1\right]$, so $\pi_{1}\left(x_{1}, x_{2}\right)$ is a unimodal and concave function and $x_{M}$ is the global maximum

3. $\forall x_{1} \in(0,1] \partial_{x_{1}} \pi_{1}\left(x_{1}, x_{2}\right)<0$, so $\pi_{1}\left(x_{1}, x_{2}\right)$ is strictly decreasing

In the first case we have that the best response to $x_{2}$ is indeed 1 , while in the third case it is 0 . In the second case we have that $0<x_{M}<1$ is the best response to $x_{2}$.

Now we prove that if the best response becomes null for some opponent's effort, it will be null for any larger effort. If the best response is null for some $\hat{x}_{2}$,we are in the third case. This implies that we also have $\partial_{x_{1}} \pi_{1}\left(x_{1}, x_{2}\right)<0$ for $x_{2}>\hat{x}_{2}$. In fact we have that

$$
\partial_{x_{1}} \pi_{1}\left(0, x_{2}\right)<\partial_{x_{1}} \pi_{1}\left(0, \hat{x}_{2}\right) \leq 0, \quad \text { with } x_{2}>\hat{x}_{2}
$$

is equivalent to

$$
\begin{aligned}
\frac{S\left(x_{2}\right) \partial_{x_{1}} S(0)}{\left(S(0)+S\left(x_{2}\right)\right)^{2}} \theta-C^{\prime}\left(x_{1}\right) & <\frac{S\left(\hat{x}_{2}\right) \partial_{x_{1}} S(0)}{\left(S(0)+S\left(\hat{x}_{2}\right)\right)^{2}} \theta-C^{\prime}\left(x_{1}\right) \\
\frac{S\left(x_{2}\right) \partial_{x_{1}} S(0)}{\left(1+S\left(x_{2}\right)\right)^{2}} & <\frac{S\left(\hat{x}_{2}\right) \partial_{x_{1}} S(0)}{\left(1+S\left(\hat{x}_{2}\right)\right)^{2}} \\
\frac{S\left(x_{2}\right)}{\left(1+S\left(x_{2}\right)\right)^{2}} & <\frac{S\left(\hat{x}_{2}\right)}{\left(1+S\left(\hat{x}_{2}\right)\right)^{2}}
\end{aligned}
$$

which is true since $x /(1+x)^{2}$ is decreasing for $x \geq 1$. Since $\partial_{x_{1}} \pi_{1}\left(0, x_{2}\right)<0$ and $\partial_{x_{1}}^{2} \pi_{1}\left(x_{1}, x_{2}\right)<0$ we can conclude that $\partial_{x_{1}} \pi_{1}\left(x_{1}, x_{2}\right)<0$ for $x_{2}>\hat{x}_{2}$ and hence the best response is null

Similarly, we have that if the best response becomes 1 for some opponent's effort, it will be 1 for any larger effort. The proof is similar to the previous case. If the best response is 1 , then we are in the first case and we can show that $\partial_{x_{1}} \pi_{1}\left(x_{1}, x_{2}\right)>0$ for $x_{2}>\hat{x}_{2}$. In fact we have that

$$
\partial_{x_{1}} \pi_{1}\left(1, x_{2}\right)>\partial_{x_{1}} \pi_{1}\left(1, \hat{x}_{2}\right) \geq 0, \quad \text { for } x_{2}>\hat{x}_{2}
$$

is equivalent to

$$
\begin{array}{ll}
\frac{S\left(x_{2}\right) \partial_{x_{1}} S(1)}{\left(S(1)+S\left(x_{2}\right){ }^{2}\right.} \theta-C^{\prime}\left(x_{1}\right) & >\frac{S\left(\hat{x}_{2}\right) \partial_{x_{1}} S(1)}{\left(S(1)+S\left(\hat{x}_{2}\right)\right)^{2}} \theta-C^{\prime}\left(x_{1}\right) \\
\frac{S\left(x_{2}\right) \partial_{x_{1}} S(1)}{\left(S(1)+S\left(x_{2}\right)\right)^{2}} & >\frac{S\left(\hat{x}_{2}\right) \partial_{x_{1}} S(1)}{\left(S(1)+S\left(\hat{x}_{2}\right)\right)^{2}} \\
\frac{S\left(x_{2}\right)}{\left(S(1)+S\left(x_{2}\right)\right)^{2}} & >\frac{S\left(\hat{x}_{2}\right)}{\left(S(1)+S\left(\hat{x}_{2}\right)\right)^{2}}
\end{array}
$$

which is true since $x /(B+x)^{2}$ is an increasing function for $x \leq B$. 
The previous considerations show that if $B R(0)=0$ (respectively $B R(0)=1$ ), then $B R \equiv 0$ (respectively $B R \equiv 0$ ), namely we have (IV) (respectively (V)).

Let us consider the remaining cases $B R(0) \neq 0$ and $B R(0) \neq 1$. Since we proved that if there exists $\hat{x} \in(0,1]$ such that $B R(\hat{x})=0$ (resp. $B R(\hat{x})=1)$, then $B R(x)=0($ resp. $B R(x)=1)$ for $x \in[\hat{x}, 1]$, we can have three possibilities

- $B R\left(x_{2}\right) \in(0,1)$ for each $x_{2} \in I=[0,1]$ and $B R(1) \in(0,1)$, which gives the best response of (I)

- there exists a $x_{a} \in(0,1]$ for which $B R\left(x_{2}\right) \in(0,1)$ for each $x_{2} \in I=\left[0, x_{a}\right)$ and $B R\left(x_{a}\right)=0$, which gives the best response of (II)

- there exists a $x_{a} \in(0,1]$ for which $B R\left(x_{2}\right) \in(0,1)$ for each $x_{2} \in I=\left[0, x_{a}\right)$ and $B R\left(x_{a}\right)=1$, which gives the best response of (III)

We notice that the three situations are identified by the behavior of $B R$ for $x_{2}=1$.

Now we study the remaining properties of BR and the corresponding Nash equilibria in (I),(II) and (III). In all cases, the best response to each $x_{2} \in I$ is the (unique) $f\left(x_{2}\right) \in(0,1)$ which solves $\partial_{x_{1}} \pi_{1}\left(f\left(x_{2}\right), x_{2}\right)=0$. This allows defining function $f: I \rightarrow(0,1)$, which, recalling the regularity properties of $S$ and $C$, thanks to the implicit function theorem, satisfies $f \in C^{2}(I)$ and

$$
\begin{aligned}
f^{\prime}\left(x_{2}\right) & =-\frac{\partial_{x_{1} x_{2}}^{2} \pi_{1}\left(f\left(x_{2}\right), x_{2}\right)}{\partial_{x_{1}}^{2} \pi_{1}\left(f\left(x_{2}\right), x_{2}\right)} \\
& =-\left(S\left(f\left(x_{2}\right)\right)-S\left(x_{2}\right)\right) \frac{\partial_{x_{2}} S\left(x_{2}\right) \partial_{x_{1}} S\left(f\left(x_{2}\right)\right)}{\left[S\left(x_{2}\right)\left(S\left(f\left(x_{2}\right)\right)+S\left(x_{2}\right)\right) \partial_{x_{1}}^{2} S\left(f\left(x_{2}\right)\right)-2 S\left(x_{2}\right)\left(\partial_{x_{1}} S\left(f\left(x_{2}\right)\right)\right)^{2}\right] \theta-C^{\prime \prime}\left(f\left(x_{2}\right)\right)}
\end{aligned}
$$

Recalling that $\partial_{x_{2}} S\left(x_{2}\right) \partial_{x_{1}} S\left(f\left(x_{2}\right)\right)>0$ and $\partial_{x_{1}}^{2} \pi_{1}\left(f\left(x_{2}\right), x_{2}\right)<0$ and we have that $f^{\prime}\left(x_{2}\right)>0$ if and only if $S\left(f\left(x_{2}\right)\right)-S\left(x_{2}\right)>0$. Then $f$ is increasing if and only if $S\left(f\left(x_{2}\right)\right)>S\left(x_{2}\right)$, namely if $f\left(x_{2}\right)>x_{2}$ and is decreasing if $f\left(x_{2}\right)<x_{2}$. Hence, if we are in case (III), we have that $f$ is strictly increasing in $I$ and indeed $\left(x^{N E}, x^{N E}\right)=(1,1)$.

In the remaining cases we have that that there exists a $x^{N E} \in(0,1)$ such that function $B R$ is strictly increasing (resp. decreasing) in $\left[0, x^{N E}\right)\left(\right.$ resp. $\left.\left(x^{N E}, 1\right]\right)$ and $f^{\prime}\left(x^{N E}\right)=0$, which gives the unimodal property of $f$ in (I) and (II). Moreover, we have that $\left(x^{N E}, x^{N E}\right)$ is a symmetric equilibrium for both (I) and (II). Such equilibrium is also the only symmetric one, because from (28) we have that in (internal) symmetric equilibria we need $f^{\prime}=0$. If more than a symmetric equilibrium existed, because of the regularity of involved functions, we would have that between two symmetric equilibria there would exist another symmetric equilibrium with $B R^{\prime} \neq 0$. Indeed, in (I) and (II) we can not have boundary symmetric equilibria.

We only have to prove that we do not have asymmetric equilibria. This is indeed true in cases (III),(IV) and $(\mathrm{V})$. In the remaining cases, let us suppose that $\left(x_{1}^{N E}, x_{2}^{N E}\right)$ is an asymmetric equilibria, namely we have $x_{1}^{N E}=B R\left(x_{2}^{N E}\right)$ and $x_{2}^{N E}=B R\left(x_{1}^{N E}\right)$ and with $x_{1}^{N E} \neq x_{2}^{N E}$. Without loss of generality, let us consider $x_{1}^{N E}>x_{2}^{N E}$. Then $B R\left(x_{2}^{N E}\right)=x_{1}^{N E}>x_{2}^{N E}$ which implies $B R^{\prime}\left(x_{2}^{N E}\right)>0$ and hence $x_{2}^{N E}<x^{N E}$. Since for $x<x^{N E}$ we have that $B R$ is an increasing function, we also have $B R\left(x_{2}^{N E}\right)<B R\left(x^{N E}\right)=x^{N E}$ and hence $x_{1}^{N E}<x^{N E}$. However, this implies that $B R^{\prime}\left(x_{1}^{N E}\right)<0$. But when $B R^{\prime}\left(x_{1}^{N E}\right)<0$ we need $x_{2}^{N E}>x^{N E}$ and this is a contradiction. 


\subsection{Proofs of Section 3.2}

We start noticing that, from (8), we have

$$
\begin{gathered}
\partial_{x_{i}} \pi_{i}\left(x_{i}, x_{j}\right)=\frac{\beta\left(\beta x_{j}+1\right)\left(\theta+\delta x_{i}+\gamma x_{j}\right)}{\left(\beta x_{i}+\beta x_{j}+2\right)^{2}}-\frac{\delta\left(\beta x_{j}+1\right)}{\beta x_{i}+\beta x_{j}+2}-1, \\
\partial_{x_{i}}^{2} \pi_{i}\left(x_{i}, x_{j}\right)=\frac{2 \beta\left(\beta x_{j}+1\right)\left(2 \delta-\beta \theta+\beta \delta x_{j}-\beta \gamma x_{j}\right)}{\left(\beta x_{i}+\beta x_{j}+2\right)^{3}} .
\end{gathered}
$$

As we shall see, all the following results can be represented, in addition to $\beta$, through the two synthetic parameters

$$
\Lambda=\beta \theta-2 \delta, \quad \Delta=\gamma-\delta .
$$

In what follows, the results are presented in terms of $\Lambda, \Delta$ and $\beta$, but can be easily rephrased in terms of $\theta, \delta$ and $\gamma$, in order to obtain the formulations reported in Section 3.2

Moreover, we set

$$
\Delta\left(x_{j}\right)=\left(\beta x_{j}+1\right)\left(\Lambda+\beta \Delta x_{j}\right),
$$

and we introduce function $x_{+}: D \rightarrow \mathbb{R}$, defined on the set $D=\left\{x_{j} \in[0,1]: \Delta\left(x_{j}\right) \geq 0\right\}$, given by

$$
x_{+}\left(x_{j}\right)=-\frac{2}{\beta}-x_{2}+\frac{\sqrt{\Delta\left(x_{j}\right)}}{\beta} .
$$

We remark that set $D$ depends on the parameters' configuration.

Lemma 5. For $\Lambda \neq \Delta$ function $x_{+}$is strictly concave, while for $\Lambda=\Delta$ it becomes

$$
x_{+}\left(x_{j}\right)=-x_{j}(1-\sqrt{\Lambda})+\frac{\sqrt{\Lambda}-2}{\beta} .
$$

In the next Lemma, we compute the best response function.

Lemma 6. The best response function $B R_{i}:[0,1] \rightarrow[0,1]$ is given by

$$
B R_{i}\left(x_{j}\right)= \begin{cases}0 & \text { if } \Delta\left(x_{j}\right)<0, \\ \min \left\{\max \left\{x_{+}\left(x_{j}\right), 0\right\}, 1\right\} & \text { otherwise, }\end{cases}
$$

where function $x_{+}$is defined by (31).

Proof. Noticing that $\Delta\left(x_{j}\right)<0$ is equivalent to $a_{1}+a_{2} \beta x_{j}<0$, we have from (29) that payoff function $\pi_{i}\left(x_{i}, x_{j}\right)$ is strictly convex with respect to $x_{i}$, so its global maximum is attained at $x_{i}=0$ and $/$ or $x_{i}=1$. Through simple algebraic manipulations, we have that

$$
\pi_{i}\left(0, x_{j}\right)-\pi_{i}\left(1, x_{j}\right)>0 \Leftrightarrow \omega=\beta^{2}\left(1-a_{2}\right) x_{j}^{2}+\beta\left(4-a_{2}-a_{1}+\beta\right) x_{j}+4+2 \beta-a_{1}>0 .
$$

Using $-a_{1} \geq \beta a_{2} x_{j}$, we have

$$
\omega>\beta^{2} x_{j}^{2}+\beta(4+\beta) x_{j}+4+2 \beta>0,
$$

where the last inequality is indeed true. This means that $B R_{i}\left(x_{j}\right)=0$.

If $\Delta\left(x_{j}\right)=0$, which means $x_{j}=-a_{1} / \beta a_{2}$, we can write

$$
\pi_{i}\left(x_{i},-\frac{a_{1}}{\beta a_{2}}\right)=-\delta \frac{a_{2}-a_{1}}{\beta a_{1}}-x_{i},
$$

which is a decreasing function and again provides $B R_{i}\left(x_{j}\right)=0$. Since when $\Delta\left(x_{j}\right)=0$ we have $x_{+}\left(x_{j}\right)<0$, we can indeed write $B R_{i}\left(x_{j}\right)=\min \left\{\max \left\{x_{+}\left(x_{j}\right), 0\right\}, 1\right\}=0$. 
Now we consider any $x_{j} \in[0,1]$ for which $\Delta\left(x_{j}\right)>0$. To find $x_{i}=\arg \max _{z \in[0,1]} \pi_{i}\left(z, x_{j}\right)$, we start noticing that, from (29), imposing $\partial_{x_{i}} \pi_{i}\left(x_{i}, x_{j}\right) \geq 0$ we obtain

$$
\begin{aligned}
-\beta^{2} x_{i}^{2}+-2 \beta\left(\beta x_{j}+2\right) x_{i}+\beta \theta-2 \delta-4 \beta x_{j}- & \beta^{2} x_{j}{ }^{2} \\
& +\beta^{2} \theta x_{j}-\beta^{2} \delta x_{j}{ }^{2}+\beta^{2} \gamma x_{j}{ }^{2}-3 \beta \delta x_{j}+\beta \gamma x_{j}-4 \geq 0 .
\end{aligned}
$$

Since $\Delta\left(x_{j}\right)>0$, the previous inequality is solved by $x_{-}\left(x_{j}\right) \leq x_{i} \leq x_{+}\left(x_{j}\right)$, where

$$
x_{ \pm}\left(x_{j}\right)=-\frac{2}{\beta}-x_{2} \pm \frac{\sqrt{\left(\beta x_{2}+1\right)\left(a_{1}+\beta a_{2} x_{j}\right)}}{\beta},
$$

which are both real and satisfy $x_{-}\left(x_{j}\right)<-1 / \beta<x_{+}\left(x_{j}\right)$. Proceeding as in the last part of the proof of Lemma 2 we easily obtain $B R_{i}\left(x_{j}\right)=\min \left\{\max \left\{x_{+}\left(x_{j}\right), 0\right\}, 1\right\}$.

If $\Delta\left(x_{j}\right)>0$ for any $x_{j} \in[0,1]$, then $B R_{i}\left(x_{j}\right)$ is indeed continuous, as well as if $\Delta\left(x_{j}\right) \leq 0$ for any $x_{j} \in[0,1]$. Conversely, if $\bar{x}_{j}=-a_{1} /\left(\beta a_{2}\right)$ belongs to $(0,1)$, we need to check the continuity of $B R_{i}$ at $x_{j}=\bar{x}_{j}$. If $a_{2}>0$, we have that $B R_{i}\left(x_{j}\right)=0$ for $x_{j} \leq \bar{x}_{j}$ and since

$$
\lim _{x_{j} \rightarrow \bar{x}_{j}^{+}} x_{+}\left(x_{j}\right)=\frac{1}{\beta}\left(\frac{a_{1}}{a_{2}}-2\right)<0,
$$

we have $\lim _{x_{j} \rightarrow \bar{x}_{j}^{+}} B R_{i}\left(x_{j}\right)=0$, so the best response function is continuous. Similarly, if $a_{2}<0$, we have that $B R_{i}\left(x_{j}\right)=0$ for $x_{j} \geq \bar{x}_{j}$ and since

$$
\lim _{x_{j} \rightarrow \bar{x}_{j}^{-}} x_{+}\left(x_{j}\right)=\frac{1}{\beta}\left(\frac{a_{1}}{a_{2}}-2\right)<0,
$$

we have $\lim _{x_{j} \rightarrow \bar{x}_{j}^{-}} B R_{i}\left(x_{j}\right)=0$, so the best response function is again continuous.

Using the previous Lemma we can prove Proposition 3

Proof of Proposition [3. Lemma 6 guarantees that when $B R_{i}\left(x_{j}\right) \in(0,1)$, then $B R_{i}\left(x_{j}\right)=x_{+}\left(x_{j}\right)$. From Lemma 5 . function $x_{+}$, is strictly concave for $a_{1} \neq a_{2}$, and then it is either strictly increasing, strictly decreasing or unimodal in its domain $D$. If $a_{1}=a_{2}$, from (32) we have that $x_{+}$can be either strictly increasing, strictly decreasing or constant (identically equal to $-1 / \beta$ if and only if $a_{1}=a_{2}=1$ ). The classification of the possible behaviors of $B R_{i}$ then follows from Lemma $\left[\right.$ essentially depending, on the values of $x_{+}$and $x_{+}^{\prime}$ at the ending points of $D \cap[0,1]$. Then $B R_{i}$ can be either strictly increasing, strictly decreasing or unimodal in those intervals in which $x_{+}\left(x_{j}\right) \in(0,1)$, constantly equal to 0 when either $x_{+}\left(x_{j}\right)<0$ or $\Delta\left(x_{j}\right)<0$ for each $x_{j} \in[0,1]$ and constantly equal to 1 when $x_{+}\left(x_{j}\right) \geq 1$ for each $x_{j} \in[0,1]$. The previous considerations together with Figure 6 which shows that each case is actually possible, allow concluding.

In Section 3.2 we reported in Figure 5 diagrams of the occurrence of each scenario of Proposition 3 for some fixed values of $\beta$ as $\beta \theta-2 \delta$ and $\gamma-\delta$ vary. In the following results we analytically specify each region shown in Figure 5 Most results are determined looking at the values of $x_{+}\left(x_{j}\right)$ and of its derivative at $x_{j}=0,1$ (provided that $x_{+}$is defined for such values). In Lemmas $7\left[10\right.$ we then study $x_{+}(0), x_{+}(1), x_{+}(0)^{\prime}$ and $x_{+}(1)^{\prime}$ on varying $\Lambda, \Delta$ and $\beta$. The proofs of Lemmas 749 are straightforward and are omitted.

Lemma 7. We have

$$
\begin{array}{lll}
\Lambda<0 & \Rightarrow & \Delta(0)<0, \\
0 \leq \Lambda<4 & \Rightarrow & x_{+}(0)<0, \\
\Lambda=4 & \Rightarrow & x_{+}(0)=0, \\
4<\Lambda<(\beta+2)^{2} & \Rightarrow & x_{+}(0) \in(0,1), \\
\Lambda=(\beta+2)^{2} & \Rightarrow & x_{+}(0)=1, \\
\Lambda>(\beta+2)^{2} & \Rightarrow & x_{+}(0)>1 .
\end{array}
$$


Lemma 8. We have

$$
\begin{array}{lll}
\Delta<-\frac{\Lambda}{\beta} & \Rightarrow & \Delta(1)<0, \\
-\frac{\Lambda}{\beta} \leq \Delta<\frac{(\beta+2)^{2}}{\beta(\beta+1)}-\frac{\Lambda}{\beta} & \Rightarrow & x_{+}(1)<0, \\
\Delta=\frac{(\beta+2)^{2}}{\beta((\beta+1)}-\frac{\Lambda}{\beta} & \Rightarrow & x_{+}(1)=0, \\
\frac{(\beta+2)^{2}}{\beta(\beta+1)}-\frac{\Lambda}{\beta}<\Delta<\frac{4(\beta+1)}{\beta}-\frac{\Lambda}{\beta} & \Rightarrow & x_{+}(1) \in(0,1), \\
\Delta=\frac{4(\beta+1)}{\beta}-\frac{\Lambda}{\beta} & \Rightarrow & x_{+}(1)=1, \\
\Delta>\frac{4(\beta+1)}{\beta}-\frac{\Lambda}{\beta} & \Rightarrow & x_{+}(1)>1 .
\end{array}
$$

Lemma 9. Let $\Delta(0)=\Lambda \geq 0$. Then we have

$$
\begin{array}{ccc}
\Delta<2 \sqrt{\Lambda}-\Lambda & \Rightarrow \quad x_{+}^{\prime}(0)<0, \\
\Delta=2 \sqrt{\Lambda}-\Lambda \quad \Rightarrow \quad x_{+}^{\prime}(0)=0, \\
\Delta>2 \sqrt{\Lambda}-\Lambda \quad \Rightarrow \quad x_{+}^{\prime}(0)>0 .
\end{array}
$$

Lemma 10. Let $\Delta(1)=\Lambda+\Delta \beta \geq 0$ and $\beta^{2}+2 \Lambda \beta+\Lambda \geq 0$. Then we have

$$
\begin{array}{lll}
\Delta<\frac{2 \beta+2 \beta^{2}-\Lambda-2 \Lambda \beta+2(\beta+1) \sqrt{\beta^{2}+2 \Lambda \beta+\Lambda}}{(2 \beta+1)^{2}} & \Rightarrow & x_{+}^{\prime}(1)<0, \\
\Delta=\frac{2 \beta+2 \beta^{2}-\Lambda-2 \Lambda \beta+2(\beta+1) \sqrt{\beta^{2}+2 \Lambda \beta+\Lambda}}{(2 \beta+1)^{2}} & \Rightarrow & x_{+}^{\prime}(1)=0, \\
\Delta>\frac{2 \beta+2 \beta^{2}-\Lambda-2 \Lambda \beta+2(\beta+1) \sqrt{\beta^{2}+2 \Lambda \beta+\Lambda}}{(2 \beta+1)^{2}} & \Rightarrow & x_{+}^{\prime}(1)>0 .
\end{array}
$$

If $\Lambda+\Delta \beta \geq 0$ and $\beta^{2}+2 \Lambda \beta+\Lambda<0$ then $x_{+}^{\prime}(1)>0$.

Proof. We have

$$
x_{+}^{\prime}(1)=\frac{a_{1}+a_{2}+2 a_{2} \beta-2 \sqrt{(\beta+1)\left(a_{1}+a_{2} \beta\right)}}{2 \sqrt{(\beta+1)\left(a_{1}+a_{2} \beta\right)}},
$$

which, thanks to the assumptions, is well-defined. To have $x_{+}^{\prime}(1)>0$ we need

$$
a_{1}+a_{2}+2 a_{2} \beta-2 \sqrt{(\beta+1)\left(a_{1}+a_{2} \beta\right)}>0,
$$

which necessarily requires that $a_{1}+a_{2}+2 a_{2} \beta>0$. If this is valid, then we can rewrite (35) as

$$
(2 \beta+1)^{2} a_{2}^{2}+2\left(a_{1}(2 \beta+1)-2 \beta(\beta+1)\right) a_{2}+a_{1}^{2}-4 a_{1}(\beta+1)>0 .
$$

If $\beta^{2}+2 a_{1} \beta+a_{1} \geq 0$, inequality (36) is solved by $a_{2}<s_{-} \vee a_{2}>s_{+}$, where

$$
s_{ \pm}=\frac{2 \beta+2 \beta^{2}-a_{1}-2 a_{1} \beta \pm 2(\beta+1) \sqrt{\beta^{2}+2 a_{1} \beta+a_{1}}}{(2 \beta+1)^{2}} .
$$

Conversely, if $\beta^{2}+2 a_{1} \beta+a_{1}<0$, we have that (36) is always fulfilled (notice that $\beta^{2}+2 a_{1} \beta+a_{1}<0$ and $a_{1}+\beta a_{2}$ imply $\left.a_{1}+a_{2}+2 a_{2} \beta>0\right)$. This provides the last claim of the Lemma.

Conversely, if

$$
\beta^{2}+2 a_{1} \beta+a_{1} \geq 0,
$$

we have that $a_{2}<s_{-}$is not possible. In fact, if $a_{1} \geq 0$, imposing both $a_{1}+a_{2}+2 a_{2} \beta>0$ and $a_{2}<s_{-}$we would need

$$
-\frac{a_{1}}{2 \beta+1}<a_{2}<\frac{2 \beta+2 \beta^{2}-a_{1}-2 a_{1} \beta-2(\beta+1) \sqrt{\beta^{2}+2 a_{1} \beta+a_{1}}}{(2 \beta+1)^{2}},
$$

which requires

$$
\beta(\beta+1)-(\beta+1) \sqrt{\beta^{2}+2 a_{1} \beta+a_{1}}>0 .
$$


$\operatorname{But}(\beta+1) \sqrt{\beta^{2}+2 a_{1} \beta+a_{1}} \geq \beta(\beta+1)$, so (38) is not possible. If $a_{1}<0$, from (37) we need $\frac{(\beta+2)^{2}}{\beta+1}<a_{1}+\beta a_{2}$, and imposing $a_{2}<s_{-}$we obtain

$$
\frac{(\beta+2)^{2}}{\beta(\beta+1)}-\frac{a_{1}}{\beta}<a_{2}<\frac{2 \beta+2 \beta^{2}-a_{1}-2 a_{1} \beta-2(\beta+1) \sqrt{\beta^{2}+2 a_{1} \beta+a_{1}}}{(2 \beta+1)^{2}},
$$

which requires

$$
20 \beta+2 \beta^{3}+31 \beta^{2}+16 \beta^{3}+2 \beta^{4}+4+2\left(\beta^{3}+4 \beta^{2}+\beta\right) \sqrt{\beta^{2}+2 a_{1} \beta+a_{1}}-\left(2 \beta^{3}+5 \beta^{2}+4 \beta+1\right) a_{1}<0
$$

which is not possible since $a_{1}<0$. Conversely, it is easy to see that

$$
-\frac{a_{1}}{2 \beta+1}<\frac{2 \beta+2 \beta^{2}-a_{1}-2 a_{1} \beta+2(\beta+1) \sqrt{\beta^{2}+2 a_{1} \beta+a_{1}}}{(2 \beta+1)^{2}}
$$

which allows concluding the part related to $x_{+}^{\prime}(1)>0$. The proof of cases $x_{+}^{\prime}(1)=0$ and $x_{+}^{\prime}(1)<0$ can be similarly handled.

Lemma 11. Let $\Lambda<4$. Then we have that there exists $x_{M} \in(0,1]$ such that $x_{+}\left(x_{M}\right)>0$ if and only if

$$
\left\{\begin{array} { l } 
{ \Lambda > \frac { 3 \beta ^ { 2 } + 8 \beta + 4 } { ( \beta + 1 ) ^ { 2 } } , } \\
{ \Delta > \Lambda - 4 + 2 \sqrt { 4 - \Lambda } , }
\end{array} \cup \left\{\begin{array}{l}
\Lambda<\frac{3 \beta^{2}+8 \beta+4}{(\beta+1)^{2}} \\
\Delta>\frac{(\beta+2)^{2}}{\beta(\beta+1)}-\frac{\Lambda}{\beta}
\end{array}\right.\right.
$$

Proof. If $a_{1}<\frac{3 \beta^{2}+8 \beta+4}{(\beta+1)^{2}}$ and $a_{2}>\frac{(\beta+2)^{2}}{\beta(\beta+1)}-\frac{a_{1}}{\beta}$, from Lemma 8 we have that $x_{+}(1)>0$, and then we can choose $x_{M}=1$. If $a_{1}>\frac{3 \beta^{2}+8 \beta+4}{(\beta+1)^{2}}$ and $a_{2}>a_{1}-4+2 \sqrt{4-a_{1}}$, we distinguish two cases. If $a_{2}>\frac{(\beta+2)^{2}}{\beta(\beta+1)}-\frac{a_{1}}{\beta}$ (notice that $\frac{(\beta+2)^{2}}{\beta(\beta+1)}-\frac{a_{1}}{\beta}>a_{1}-4+2 \sqrt{4-a_{1}}$ for $a_{1}>\frac{3 \beta^{2}+8 \beta+4}{(\beta+1)^{2}}$ ), we can again choose $x_{M}=1$ thanks to Lemma 8. Conversely, if $a_{1}-4+2 \sqrt{4-a_{1}}<a_{2} \leq \frac{(\beta+2)^{2}}{\beta(\beta+1)}-\frac{a_{1}}{\beta}$ we can choose

$$
x_{M}=\frac{-\left(a_{1}+a_{2}\right)\left(1-a_{2}\right)+\left(a_{1}-a_{2}\right) \sqrt{1-a_{2}}}{2 a_{2} \beta\left(1-a_{2}\right)},
$$

which is well defined since for $a_{1}>\frac{3 \beta^{2}+8 \beta+4}{(\beta+1)^{2}}$ we have

$$
\frac{(\beta+2)^{2}}{\beta(\beta+1)}-\frac{a_{1}}{\beta}<1
$$

Noticing that for $a_{1}>\frac{3 \beta^{2}+8 \beta+4}{(\beta+1)^{2}}$ we have $a_{2}>a_{1}$, we obtain

$$
x_{+}\left(x_{M}\right)=\frac{-\left(a_{1}-a_{2}\right)\left(1-a_{2}\right)+\left(a_{1}-3 a_{2}\right) \sqrt{1-a_{2}}}{2 a_{2} \beta \sqrt{1-a_{2}}} .
$$

A direct check shows that under the previous conditions we have $x_{+}\left(x_{M}\right)>0$.

The converse can be proved by contradiction, considering each case obtained by negating (39) and showing that for each of them $\sup _{x \in(0,1] \cap\left\{x_{j}: \Delta\left(x_{j}\right) \geq 0\right\}} x_{+}<0$. It is a simple but very long check, so we do not provide details.

In each of the following proposition we provide sufficient conditions on the parameters for the occurrence of each best response shape. We remark that since such scenarios are mutually exclusive by definition and since the conditions used in Propositions 10, 14 provide a partition of $(\Lambda, \Delta, \beta)$ space, such conditions are actually necessary, too. 
Proposition 10. Best response functions of games $\Gamma \in \Gamma^{L S O}$ are strictly non-increasing if

$$
\left\{\begin{array} { l } 
{ 4 < \Lambda < ( \beta + 2 ) ^ { 2 } , } \\
{ \Delta \leq 2 \sqrt { \Lambda } - \Lambda , }
\end{array} \quad \text { or } \quad \left\{\begin{array}{l}
\Lambda \geq(\beta+2)^{2}, \\
\Delta<\frac{4(\beta+1)-\Lambda}{\beta},
\end{array}\right.\right.
$$

which corresponds to the yellow regions in Figure 5 .

Proof. Let us consider the former couple of inequalities. We have that

a) $4<a_{1}<(\beta+2)^{2} \Rightarrow x_{+}(0) \in(0,1)$ (Lemma 7);

b) $a_{2} \leq 2 \sqrt{a_{1}}-a_{1} \Rightarrow x_{+}^{\prime}(0) \leq 0$ (Lemma 9, notice that, from a), we have $a_{1}>4>0$, and then this condition is well-defined).

Then we have $B R_{i}(0) \in(0,1)$ and $B R_{i}^{\prime}(0) \leq 0$. This means that there exists $b \in(0,1]$ such that $B R_{i}\left(x_{j}\right)=x_{+}\left(x_{j}\right)$ for $x_{j} \in[0, b]$ and $B R_{i}\left(x_{j}\right)=0$ for $x_{j} \in(b, 1]$, where this latter interval may be empty. Since $x_{+}$is strictly concave, $x_{+}^{\prime}(0)=B R_{i}^{\prime}(0) \leq 0$ means that $x_{+}^{\prime}\left(x_{j}\right)=B R_{i}^{\prime}\left(x_{j}\right)<0$ for $x_{j} \in[0, b)$. Moreover, we indeed have $B R_{i}^{\prime}\left(x_{j}\right)=0$ for $x_{j} \in(b, 1]$.

Now we consider the latter couple of inequalities. We have that

a) $a_{1} \geq(\beta+2)^{2} \Rightarrow x_{+}(0) \geq 1$ (Lemma 7);

b) $a_{2}<\frac{4(\beta+1)-a_{1}}{\beta} \Rightarrow$ either $x_{+}(1)<1$ or $\Delta(1)<0$ (Lemma 8).

Then we have $B R_{i}(0)=1$ and $B R_{i}(1) \in[0,1)$. This means that exist $0 \leq b<c \leq 1$ such that $B R_{i}\left(x_{j}\right)=1$ for $x_{j} \in[0, b], B R_{i}\left(x_{j}\right)=x_{+}\left(x_{j}\right)$ for $x_{j} \in(b, c]$ and $B R_{i}\left(x_{j}\right)=0$ for $x_{j} \in(c, 1]$. Notice that this latter interval is empty if $B R_{i}(1)>0$. Moreover, we must have $B R_{i}^{\prime}\left(x_{j}\right)=x_{+}^{\prime}\left(x_{j}\right)<0$ for $x_{j} \in(b, c)$, $B R_{i}^{\prime}\left(x_{j}\right)=0$ for $x_{j} \in[0, b) \cup(c, 1]$.

Proposition 11. Best response functions of games $\Gamma \in \Gamma^{L S O}$ are constantly equal to 1 if

$$
\left\{\begin{array}{l}
\Lambda \geq(\beta+2)^{2} \\
\Delta \geq \frac{4(\beta+1)-\Lambda}{\beta},
\end{array}\right.
$$

which defines the cyan region of Figure 5 .

Proof. We have that

a) $a_{1} \geq(\beta+2)^{2} \Rightarrow x_{+}(0) \geq 1$ (Lemma 7);

b) $a_{2} \geq \frac{4(\beta+1)-a_{1}}{\beta} \Rightarrow x_{+}(1) \geq 1$ (Lemma 8).

Then we have $B R_{i}(0)=B R_{i}(1)=1$. Since $B R_{i}$ is strictly concave, we can not have $B R_{i}\left(x_{j}\right)<1$ for any $x_{j} \in(0,1)$. Then $B R_{i} \equiv 1$, which allows concluding.

Proposition 12. Best response functions of games $\Gamma \in \Gamma^{L S O}$ are strictly non-decreasing if

$$
\left\{\begin{array}{l}
4 \leq \Lambda<(\beta+2)^{2}, \\
\Delta \geq \min \left\{\frac{2 \beta+2 \beta^{2}-\Lambda-2 \Lambda \beta+2(\beta+1) \sqrt{\beta^{2}+2 \Lambda \beta+\Lambda}}{(2 \beta+1)^{2}}, \frac{4(\beta+1)-\Lambda}{\beta}\right\},
\end{array}\right.
$$

or

$$
\left\{\begin{array}{l}
\frac{3 \beta^{2}+8 \beta+4}{(\beta+1)^{2}}<\Lambda<4 \\
\Delta \geq \frac{2 \beta+2 \beta^{2}-\Lambda-2 \Lambda \beta+2(\beta+1) \sqrt{\beta^{2}+2 \Lambda \beta+\Lambda}}{(2 \beta+1)^{2}}
\end{array}\right.
$$


or

$$
\left\{\begin{array}{l}
\Lambda<\frac{3 \beta^{2}+8 \beta+4}{(\beta+1)^{2}} \\
\Delta>\frac{(\beta+2)^{2}}{\beta(\beta+1)}-\frac{\Lambda}{\beta}
\end{array}\right.
$$

which define the green region of Figure 5 .

Proof. Let us consider the first couple of inequalities and suppose that

$$
\frac{2 \beta+2 \beta^{2}-a_{1}-2 a_{1} \beta+2(\beta+1) \sqrt{\beta^{2}+2 a_{1} \beta+a_{1}}}{(2 \beta+1)^{2}} \leq \frac{4(\beta+1)-a_{1}}{\beta} .
$$

We have that

a) $4 \leq a_{1}<(\beta+2)^{2} \Rightarrow x_{+}(0) \in[0,1)$ (Lemma 7);

b) $a_{2} \geq \frac{2 \beta+2 \beta^{2}-a_{1}-2 a_{1} \beta+2(\beta+1) \sqrt{\beta^{2}+2 a_{1} \beta+a_{1}}}{(2 \beta+1)^{2}} \Rightarrow x_{+}^{\prime}(1) \geq 0$ (Lemma 10, notice that, from a), we have that $\left.\beta^{2}+2 a_{1} \beta+a_{1}>0\right)$.

Then we have $B R_{i}(0) \in[0,1)$ and $B R_{i}^{\prime}(1) \geq 0$. If $B R_{i}(0) \in(0,1)$ and $B R_{i}^{\prime}(1) \geq 0$, we have that $x_{+}$is well-defined for each $x_{j} \in[0,1]$ and, by concavity, that $x_{+}^{\prime}\left(x_{j}\right)>0$ for any $x_{j} \in(0,1)$. Then, there exists $0<b \leq 1$ such that $B R_{i}\left(x_{j}\right)=x_{+}\left(x_{j}\right)$ for $x_{j} \in[0, b]$ and $B R_{i}\left(x_{j}\right)=1$ for $x_{j} \in(b, 1]$. Notice that $(b, 1]$ may be empty. This means that we have $B R_{i}^{\prime}\left(x_{j}\right)=x_{+}^{\prime}\left(x_{j}\right)>0$ for $x_{j} \in[0, b)$ and indeed we have $B R_{i}^{\prime}\left(x_{j}\right)=0$ for $x_{j} \in(b, 1]$

Notice that for $a_{1}=4$ (for which $B R_{i}(0)=x_{+}(0)=0$ ) inequality (40) becomes

$$
\frac{(2 \beta+2) \sqrt{\beta^{2}+8 \beta+4}+2 \beta^{2}-6 \beta-4}{(2 \beta+1)^{2}}<4
$$

which is true since a simple computation allows showing that its l.h.s. belongs to $(0,1)$ for $\beta>0$.

Since $x_{+}(0)^{\prime}=a_{2} / 4=B R_{i}^{\prime}(0)$, this guarantees that we are not in the case of constantly null best response function. Recalling $B R_{i}^{\prime}(1) \geq 0$, this allows concluding.

Conversely, if (40) is false, we have that

a) $4 \leq a_{1}<(\beta+2)^{2} \Rightarrow x_{+}(0) \in[0,1)$ (Lemma 7);

b) $a_{2} \geq \frac{4(\beta+1)-a_{1}}{\beta} \Rightarrow x_{+}(1) \geq 1$ (Lemma 8).

This means that $x_{+}$is well-defined for each $[0,1]$ and, thanks to the concavity of $x_{+}$, that there exists $0<b<1$ such that $B R_{i}\left(x_{j}\right)=x_{+}\left(x_{j}\right)$ for $x_{j} \in[0, b]$ and $B R_{i}\left(x_{j}\right)=1$ for $x_{j} \in(b, 1]$. By concavity, we must also have $B R_{i}^{\prime}\left(x_{j}\right)=x_{+}^{\prime}\left(x_{j}\right)>0$ for $x_{j} \in[0, b)$, and we indeed have $B R_{i}^{\prime}\left(x_{j}\right)=0$ for $x_{j} \in(b, 1]$.

Let us consider the second couple of inequalities. We have that

a) $a_{1}<4 \Rightarrow$ either $x_{+}(0)<0$ or $\Delta(0)<0$ (Lemma 7);

b) $a_{2} \geq \frac{2 \beta+2 \beta^{2}-a_{1}-2 a_{1} \beta+2(\beta+1) \sqrt{\beta^{2}+2 a_{1} \beta+a_{1}}}{(2 \beta+1)^{2}} \Rightarrow x_{+}^{\prime}(1) \geq 0$ (Lemma 10 notice that, from $\left(3 \beta^{2}+8 \beta+\right.$ 4) $/(\beta+1)^{2}<a_{1}$, we have that $\beta^{2}+2 a_{1} \beta+a_{1}>0$.).

Moreover, a simple check shows that if $\frac{3 \beta^{2}+8 \beta+4}{(\beta+1)^{2}}<a_{1}$ we have

$$
\frac{(\beta+2)^{2}}{\beta(\beta+1)}-\frac{a_{1}}{\beta}<\frac{2 \beta+2 \beta^{2}-a_{1}-2 a_{1} \beta+2(\beta+1) \sqrt{\beta^{2}+2 a_{1} \beta+a_{1}}}{(2 \beta+1)^{2}},
$$

and so, from Lemma 8 , we also have that $x_{+}(1)>0$. 
We then have $B R_{i}(0)=0$ and $B R_{i}(1)>0$. Moreover, by the concavity of $x_{+}$, from $x_{+}^{\prime}(1)>0$ we have $B R_{i}^{\prime}\left(x_{j}\right) \geq 0$. This means that there exist $0<b<c \leq 1$ such that $B R_{i}\left(x_{j}\right)=0$ for $x_{j} \in[0, b)$ and $B R_{i}\left(x_{j}\right)=x_{+}\left(x_{j}\right)$ for $x_{j} \in(b, c]$ and $B R_{i}\left(x_{j}\right)=1$ for $x_{j} \in(c, 1]$. Notice that $(c, 1]$ can be empty. Then we have that $B R_{i}^{\prime}\left(x_{j}\right)=0$ for $x_{j} \in[0, b) \cup(c, 1]$ and $B R_{i}^{\prime}\left(x_{j}\right)=x_{+}^{\prime}\left(x_{j}\right)>0$ for $x_{j} \in(b, c)$.

Let us consider the last couple of inequalities. We have that

a) $a_{1}<\frac{3 \beta^{2}+8 \beta+4}{(\beta+1)^{2}}<4 \Rightarrow$ either $x_{+}(0)<0$ or $\Delta(0)<0$ (Lemma 17);

b) $a_{2}>\frac{(\beta+2)^{2}}{\beta(\beta+1)}-\frac{a_{1}}{\beta} \Rightarrow x_{+}(1)>0($ Lemma 8$)$.

A simple check shows that if $a_{2}>\frac{(\beta+2)^{2}}{\beta(\beta+1)}-\frac{a_{1}}{\beta}$ then $a_{1}+a_{2} \beta \geq 0$. If which, Lemma 10, guarantees that $x_{+}^{\prime}(1) \geq 0$. If $\beta^{2}+2 a_{1} \beta+a_{1}<0$, then, from Lemma 10 we have $x_{+}^{\prime}(1)>0$. Conversely, if $\beta^{2}+2 a_{1} \beta+a_{1} \geq 0$, since fro $\frac{3 \beta^{2}+8 \beta+4}{(\beta+1)^{2}}>a_{1}$ we have

$$
\frac{(\beta+2)^{2}}{\beta(\beta+1)}-\frac{a_{1}}{\beta}>\frac{2 \beta+2 \beta^{2}-a_{1}-2 a_{1} \beta+2(\beta+1) \sqrt{\beta^{2}+2 a_{1} \beta+a_{1}}}{(2 \beta+1)^{2}},
$$

from Lemma 10 we have $x_{+}^{\prime}(1) \geq 0$. Proceeding as for the second couple of inequalities we can conclude.

Proposition 13. Best response functions of games $\Gamma \in \Gamma^{L S O}$ are hump-shaped if

$$
\left\{\begin{array}{l}
4 \leq \Lambda<(\beta+2)^{2}, \\
2 \sqrt{\Lambda}-\Lambda<\Delta<\min \left\{\frac{2 \beta+2 \beta^{2}-\Lambda-2 \Lambda \beta+2(\beta+1) \sqrt{\beta^{2}+2 \Lambda \beta+\Lambda}}{(2 \beta+1)^{2}}, \frac{4(\beta+1)-\Lambda}{\beta}\right\},
\end{array}\right.
$$

or

$$
\left\{\begin{array}{l}
\frac{3 \beta^{2}+8 \beta+4}{(\beta+1)^{2}}<\Lambda<4 \\
\Lambda-4+2 \sqrt{4-\Lambda}<\Delta<\frac{2 \beta+2 \beta^{2}-\Lambda-2 \Lambda \beta+2(\beta+1) \sqrt{\beta^{2}+2 \Lambda \beta+\Lambda}}{(2 \beta+1)^{2}}
\end{array}\right.
$$

which define the white region of Figure 5 .

Proof. Let us consider the former couple of inequalities. We have that

a) $4 \leq a_{1}<(\beta+2)^{2} \Rightarrow x_{+}(0) \in[0,1)$ (Lemma 17);

b) $2 \sqrt{a_{1}}-a_{1}<a_{2} \Rightarrow x_{+}^{\prime}(0)>0$ (Lemma 9 notice that, from a), we have $a_{1} \geq 4>0$ );

c) $a_{2}<\min \left\{\frac{2 \beta+2 \beta^{2}-a_{1}-2 a_{1} \beta+2(\beta+1) \sqrt{\beta^{2}+2 a_{1} \beta+a_{1}}}{(2 \beta+1)^{2}}, \frac{4(\beta+1)-a_{1}}{\beta}\right\} \Rightarrow\left\{\begin{array}{l}x_{+}^{\prime}(1)<0 \\ x_{+}(1)<1\end{array} \quad\right.$ (Lemma 8 and 10 notice that, from a), we have $\beta^{2}+2 a_{1} \beta+a_{1}>0$ ).

We have $B R_{i}(0) \in[0,1), B R_{i}^{\prime}(0)>0$ and $B R_{i}^{\prime}(1) \leq 0$. If $B R_{i}^{\prime}(1)<0$ we indeed have a hump-shaped best response function, since we have at least a point $\left(x_{j}=0\right)$ at which $B R_{i}$ is strictly increasing and at least a point $\left(x_{j}=1\right)$ at which $B R_{i}$ is strictly decreasing. Conversely, if $B R_{i}(1)=0$, thanks to the continuity of $B R_{i}$ and the regularity of $x_{+}$, we must have that $B R_{i}^{\prime}\left(x_{j}\right)<0$ for some $x_{j}$.

Let us consider the latter couple of inequalities. We have that

a) $a_{1}<4 \Rightarrow$ either $\Delta<0$ or $x_{+}(0)<0$ (Lemma 7$)$

b) $\left\{\begin{array}{l}a_{1}-4+2 \sqrt{4-a_{1}}<a_{2} \\ a_{1}>0\end{array} \Rightarrow x_{+}\left(x_{j}\right)>0\right.$ for some $x_{j}$ (Lemma 11) 
c) $a_{2}<\frac{2 \beta+2 \beta^{2}-a_{1}-2 a_{1} \beta+2(\beta+1) \sqrt{\beta^{2}+2 a_{1} \beta+a_{1}}}{(2 \beta+1)^{2}} \Rightarrow x_{+}^{\prime}(1)<0$ (Lemma 10 notice that $a_{1}>0$ guarantees that $\left.\beta^{2}+2 a_{1} \beta+a_{1}>0\right)$.

Notice that b) and c) are simultaneously possible only if

$$
\frac{3 \beta^{2}+8 \beta+4}{(\beta+1)^{2}}<a_{1} .
$$

We have that there exist $0<b<c \leq 1$ such that $B R_{i}\left(x_{j}\right)=0$ for $x_{j} \in[0, b), B R_{i}\left(x_{j}\right)=x_{+}\left(x_{j}\right) \in(0,1)$ for $x_{j} \in(b, c)$ and $B R_{i}\left(x_{j}\right)=0$ for $x_{j} \in(c, 1]$. Thanks to the continuity of $B R_{i}$, it must be unimodal in $(b, c)$.

Proposition 14. Best response functions of games $\Gamma \in \Gamma^{L S O}$ are constantly equal to 0 if

$$
\left\{\begin{array}{l}
\frac{3 \beta^{2}+8 \beta+4}{(\beta+1)^{2}} \leq \Lambda \leq 4 \\
\Delta \leq \Lambda-4+2 \sqrt{4-\Lambda}
\end{array}\right.
$$

or

which define the blue region of Figure 5 .

$$
\left\{\begin{array}{l}
\Lambda \leq \frac{3 \beta^{2}+8 \beta+4}{(\beta+1)^{2}}, \\
\Delta \leq \frac{(\beta+2)^{2}}{\beta(\beta+1)}-\frac{\Lambda}{\beta} .
\end{array}\right.
$$

Proof. From Lemma 11, in both cases, $x_{+}$can not assume strictly positive values for $x_{j} \in(0,1] \cap\left\{x_{j}\right.$ : $\left.\Delta\left(x_{j}\right) \geq 0\right\}$. This concludes the proof.

To prove Proposition 4 Corollary 3 and to provide analytical justification of Figure 9, we need several preliminary results. In what follows we introduce the set $N E$ of the possible Nash equilibria, which, as the best response function, depends on $\Lambda, \Delta, \beta$. To avoid burdening the notation, we do not explicitly write such dependence.

Firstly we study the possible internal (i.e. belonging to $\left.(0,1)^{2}\right)$ equilibria of games $\Gamma \in \Gamma^{L S O}$. We omit the proof of the next Lemma, which can be obtained (after some long but simple computations) by solving the system of $x_{i}=x_{+}\left(x_{j}\right)$ and $x_{j}=x_{+}\left(x_{i}\right)$.

Lemma 12. Let $\Lambda \neq 4$ and $\Delta \neq 4$. The only possible intersections between $x_{i}=x_{+}\left(x_{j}\right)$ and $x_{j}=x_{+}\left(x_{i}\right)$, belonging to $(0,1)$ are $\left(x_{i}, x_{j}\right) \in\left\{\left(x_{I S}^{N E}, x_{I S}^{N E}\right),\left(x_{1, A S}^{N E}, x_{2, A S}^{N E}\right),\left(x_{2, A S}^{N E}, x_{1, A S}^{N E}\right)\right\}$, where $x_{I S}^{N E}, x_{1, A S}^{N E}$ and $x_{2, A S}^{N E}$ are defined in (10) and (11).

As a consequence of the previous Lemma, we have that the only possible elements of set $N E$ can be

- the internal equilibria $\left(x_{I S}^{N E}, x_{I S}^{N E}\right),\left(x_{1, A S}^{N E}, x_{2, A S}^{N E}\right),\left(x_{2, A S}^{N E}, x_{1, A S}^{N E}\right)$;

- the corner equilibria $(0,0),(1,1),(0,1),(1,0)$;

- the boundary equilibria $(\bar{x}, 0),(0, \bar{x}),(\bar{x}, 1),(1, \bar{x})$ with $\bar{x} \in(0,1)$.

In what follows, when we speak of $x_{I S}^{N E}, x_{1, A S}^{N E}$ and $x_{2, A S}^{N E}$, we mean that they are really internal. Indeed, expressions provided by (10) and (11) can assume also values not belonging to $(0,1)$, but in this case $\left(x_{I S}^{N E}, x_{I S}^{N E}\right),\left(x_{1, A S}^{N E}, x_{2, A S}^{N E}\right),\left(x_{2, A S}^{N E}, x_{1, A S}^{N E}\right)$ "become" boundary or corner equilibria. Moreover, when we speak of $x_{1, A S}^{N E}$ and $x_{2, A S}^{N E}$ we mean that they are really asymmetric, i.e. that $x_{1, A S}^{N E} \neq x_{2, A S}^{N E}$. We notice that a simple computation shows that if $x_{1, A S}^{N E}=x_{2, A S}^{N E}$, then $\left(x_{1, A S}^{N E}, x_{2, A S}^{N E}\right)$ and $\left(x_{2, A S}^{N E}, x_{1, A S}^{N E}\right)$ both coincide with the symmetric equilibrium $\left(x_{I S}^{N E}, x_{I S}^{N E}\right)$.

Proposition 4 and consequently Corollary 3 , follows from the previous Propositions and Lemma by simple geometrical considerations.

In the next Lemma we provide conditions under which each of the previous equilibria actually occurs. 
Lemma 13. We have that

a) $B R_{i}(0)=0$ if and only if $\Lambda \leq 4$;

b) $B R_{i}(1)=1$ if and only if $\Delta \geq \frac{4(\beta+1)}{\beta}-\frac{\Lambda}{\beta}$;

c) $x_{I S} \in(0,1)$ if and only if

$$
\left\{\begin{array} { l } 
{ \Lambda < 4 , } \\
{ \Delta > \frac { 4 ( \beta + 1 ) } { \beta } - \frac { \Lambda } { \beta } , }
\end{array} \cup \left\{\begin{array}{l}
\Lambda>4, \\
\Delta<\frac{4(\beta+1)}{\beta}-\frac{\Lambda}{\beta},
\end{array}\right.\right.
$$

d) $\left\{\begin{array}{l}x_{1, A S}^{N E} \in(0,1), \\ x_{2, A S}^{N E} \in(0,1), \\ x_{1, A S}^{N E} \neq x_{2, A S}^{N E},\end{array}\right.$ if and only if

$$
\left\{\begin{array}{l}
-\Delta \frac{\sqrt{\frac{\Delta+4}{\Delta}}+1}{1-\sqrt{\frac{\Delta+4}{\Delta}}}<\Lambda<-\Delta \frac{1+2 \beta-\sqrt{\frac{\Delta+4}{\Delta}}}{1+\sqrt{\frac{\Delta+4}{\Delta}}} \\
\Delta<-4
\end{array}\right.
$$

e) $\left\{\begin{array}{l}B R_{i}(0) \in(0,1), \\ B R_{j}\left(B R_{i}(0)\right)=0,\end{array}\right.$ if and only if

$$
\left\{\begin{array}{l}
\Delta \leq-\Lambda \frac{1+\sqrt{\Lambda}}{\Lambda-1}, \\
4<\Lambda<(\beta+2)^{2},
\end{array}\right.
$$

f) $\left\{\begin{array}{l}B R_{i}(0)=1, \\ B R_{j}(1)=0,\end{array}\right.$ if and only if

$$
\left\{\begin{array}{l}
\Delta \leq \frac{(\beta+2)^{2}}{\beta(\beta+1)}-\frac{\Lambda}{\beta} \\
\Lambda \geq(\beta+2)^{2}
\end{array}\right.
$$

g) $\left\{\begin{array}{l}B R_{i}(1) \in(0,1), \\ B R_{j}\left(B R_{i}(1)\right)=1,\end{array}\right.$ if and only if

$$
\left\{\begin{array} { l } 
{ - \Delta \frac { 1 + 2 \beta - \sqrt { \frac { \Delta + 4 } { \Delta } } } { 1 + \sqrt { \frac { \Delta + 4 } { \Delta } } } \leq \Lambda < \frac { 4 ( \beta + 1 ) } { \beta } - \frac { \Lambda } { \beta } , } \\
{ - \frac { ( \beta + 2 ) ^ { 2 } } { \beta + 1 } < \Delta < - 4 , }
\end{array} \cup \left\{\begin{array}{l}
\frac{(\beta+2)^{2}}{\beta(\beta+1)}-\frac{\Lambda}{\beta}<\Lambda<\frac{4(\beta+1)}{\beta}-\frac{\Lambda}{\beta}, \\
\Delta \leq-\frac{(\beta+2)^{2}}{\beta+1} .
\end{array}\right.\right.
$$

Proof. a,b) The proof is straightforward.

c) It is sufficient to solve $0<x_{I S}<1$. From

$$
0<\frac{a_{1}-4}{\beta\left(4-a_{2}\right)}<1
$$

we have

$$
\left\{\begin{array} { l } 
{ a _ { 1 } - 4 < 0 , } \\
{ 4 - a _ { 2 } < 0 , } \\
{ a _ { 2 } > \frac { 4 ( \beta + 1 ) } { \beta } - \frac { a _ { 1 } } { \beta } , }
\end{array} \cup \left\{\begin{array}{l}
a_{1}-4>0, \\
4-a_{2}>0, \\
a_{2}<\frac{4(\beta+1)}{\beta}-\frac{a_{1}}{\beta} .
\end{array}\right.\right.
$$


Noticing that in each system the first and the last conditions imply the second ones allows concluding.

d) To have $x_{I A S, 1} \in(0,1)$ and $x_{I A S, 2} \in(0,1)$ we must solve

$$
\left\{\begin{array}{l}
-\frac{a_{1}+a_{2}+\left(a_{2}-a_{1}\right) \sqrt{\frac{a_{2}+4}{a_{2}}}}{2 a_{2} \beta}>0, \\
-\frac{a_{1}+a_{2}-\left(a_{2}-a_{1}\right) \sqrt{\frac{a_{2}+4}{a_{2}}}}{2 a_{2} \beta}>0, \\
-\frac{a_{1}+a_{2}+\left(a_{2}-a_{1}\right) \sqrt{\frac{a_{2}+4}{a_{2}}}}{2 a_{2} \beta}<1, \\
-\frac{a_{1}+a_{2}-\left(a_{2}-a_{1}\right) \sqrt{\frac{a_{2}+4}{a_{2}}}}{2 a_{2} \beta}<1,
\end{array}\right.
$$

in which we must require that either $a_{2} \leq-4$ or $a_{2}>0$, to guarantee the positivity of $\left(a_{2}+4\right) / a_{2}$. However, we must exclude $a_{2}>0$. If in fact we assume $a_{2}>0$, adding the first two inequalities we find

$$
-\frac{a_{1}+a_{2}}{a_{2} \beta}>0
$$

and then $a_{1}+a_{2}<0$, which, since we assumed $a_{2}>0$, necessarily requires $a_{1}<0$. Since $\sqrt{\frac{a_{2}+4}{a_{2}}}>1$, we have

$$
-\left(a_{1}+a_{2}\right)-\left(a_{2}-a_{1}\right) \sqrt{\frac{a_{2}+4}{a_{2}}}<-\left(a_{1}+a_{2}\right)-\left(a_{2}-a_{1}\right)=-2 a_{2}<0,
$$

and then the second inequality is always false for $a_{2}>0$. Similarly, we must also exclude $a_{2}=-4$, as in this case we have $x_{I A S, 1}=x_{I A S, 2}=x_{I S}$.

We then suppose $a_{2}<-4$. The previous system can be rewritten as

$$
\left\{\begin{array}{l}
a_{1}+a_{2}+\left(a_{2}-a_{1}\right) \sqrt{\frac{a_{2}+4}{a_{2}}}>0 \\
a_{1}+a_{2}-\left(a_{2}-a_{1}\right) \sqrt{\frac{a_{2}+4}{a_{2}}}>0 \\
a_{1}+a_{2}+\left(a_{2}-a_{1}\right) \sqrt{\frac{a_{2}+4}{a_{2}}}<-2 a_{2} \beta \\
a_{1}+a_{2}-\left(a_{2}-a_{1}\right) \sqrt{\frac{a_{2}+4}{a_{2}}}<-2 a_{2} \beta .
\end{array}\right.
$$

Adding the first two inequalities we find $a_{1}+a_{2}>0$, which requires $a_{1}>4$. After noticing that the second and the third inequalities are less restrictive than, respectively, the first and the fourth ones and that $\sqrt{\frac{a_{2}+4}{a_{2}}}<1$ for $a_{2} \leq-4$, we can easily conclude by solving the first and the fourth inequalities with respect to $a_{1}$.

e) We have that $B R_{i}(0) \in(0,1)$ if and only if $x_{+}(0) \in(0,1)$, which immediately provides $4<a_{1}<(\beta+2)^{2}$. We have that $B R_{j}\left(B R_{i}(0)\right)=0$ if and only if $\Delta\left(B R_{i}(0)\right)=\left(\sqrt{a_{1}}-1\right)\left(a_{1}+a_{2}\left(\sqrt{a_{1}}-2\right)\right)<0$ or

$$
x_{+}\left(x_{+}(0)\right)=\frac{\sqrt{\left(\sqrt{a_{1}}-1\right)\left(a_{1}+a_{2}\left(\sqrt{a_{1}}-2\right)\right)}-\sqrt{a_{1}}}{\beta} \leq 0 .
$$

Condition $\Delta\left(B R_{i}(0)\right)<0$ is fulfilled for $a_{2}<-a_{1} /\left(\sqrt{a_{1}}-2\right)$ while $x_{+}\left(x_{+}(0)\right) \leq 0$ leads to $-a_{1} /\left(\sqrt{a_{1}}-2\right) \leq$ $a_{2} \leq-a_{1} /\left(\sqrt{a_{1}}-1\right)$. Noticing that this last inequality is always satisfied by some $a_{2}$, we can conclude that we need $a_{2} \leq-a_{1} /\left(\sqrt{a_{1}}-1\right)=-a_{1}\left(\sqrt{a_{1}}+1\right) /\left(a_{1}-1\right)$.

f) $B R_{i}(0)=1$ is equivalent to $x_{+}(1) \geq 0$, i.e. $a_{1} \geq(\beta+2)^{2} . B R_{j}(1)=0$ is equivalent to either $\Delta(1)<0$ (i.e. $\left.a_{1}+\beta a_{2}<0\right)$ or

$$
x_{+}(1) \leq 0 \Leftrightarrow\left\{\begin{array}{l}
a_{1}+\beta a_{2} \geq 0 \\
-\beta-2+\sqrt{(\beta+1)\left(a_{1}+\beta a_{2}\right)} \leq 0
\end{array} \quad \Leftrightarrow 0 \leq a_{1}+\beta a_{2} \leq \frac{(\beta+2)^{2}}{\beta+1},\right.
$$

which allows concluding.

g) To have $B R_{i}(1) \in(0,1)$ we need $x_{+}(1) \in(0,1)$. A direct computation shows that this is equivalent to

$$
\frac{(\beta+2)^{2}}{(\beta+1)}-\beta a_{2}<a_{1}<4(\beta+1)-\beta a_{2} \text {. }
$$


Notice that if $a_{1} \leq 4$, from point a), we would have that $B R_{i}(0)=0$. To have $B R_{j}\left(B R_{i}(1)\right)=1$ we would need that $x_{j}=B R_{i}\left(x_{j}\right)$ has two solutions $x_{j} \in(0,1)$, which is not possible. Then we must have $a_{1}>4$.

Condition $B R_{j}\left(B R_{i}(1)\right) \geq 1$ is equivalent to $x_{+}\left(x_{+}(1)\right) \geq 1$, i.e.

$$
\left\{\begin{array}{l}
\sqrt{\left(a_{1}-a_{2}\left(\beta-\sqrt{(\beta+1)\left(a_{1}+a_{2} \beta\right)}+2\right)\right)\left(\sqrt{(\beta+1)\left(a_{1}+a_{2} \beta\right)}-\beta-1\right)}-\sqrt{(\beta+1)\left(a_{1}+a_{2} \beta\right)} \geq 0, \\
a_{1}+a_{2} \beta \geq 0 .
\end{array}\right.
$$

Notice that the argument under the square root in the first inequality is positive if the inequality is satisfied, so we do not need to add further conditions on it.

Let us rewrite introducing the linear transformation $x=a_{1}+\beta a_{2}$. Elevating each member of the first inequality to the second and rearranging terms we arrive to $k_{1} a_{1}+k_{2} \geq 0$, where

$$
\begin{gathered}
k_{1}=(\sqrt{x(\beta+1)}-\beta-1)(2 \beta+2-\sqrt{x(\beta+1)}), \\
k_{2}=x((1+\beta)(x+2)-(3+2 \beta) \sqrt{x(\beta+1)}) .
\end{gathered}
$$

Notice that both $k_{1}$ and $k_{2}$ are strictly positive for $x \in\left(\frac{(\beta+2)^{2}}{(\beta+1)}, 4(\beta+1)\right)$. Then we have

$$
a_{1} \geq-\frac{k_{2}}{k_{1}}=x \frac{x-\beta+\beta x-1+\beta \sqrt{x(\beta+1)}}{(\beta+1)(x-\beta-1)} .
$$

The last inequality can be rewritten as

$$
a_{1} \geq\left(a_{1}+\beta a_{2}\right) \frac{a_{1}+\beta a_{2}-\beta+\beta\left(a_{1}+\beta a_{2}\right)-1+\beta \sqrt{\left(a_{1}+\beta a_{2}\right)(\beta+1)}}{(\beta+1)\left(a_{1}+\beta a_{2}-\beta-1\right)},
$$

from which we have

$$
\frac{-\beta\left[(\beta+1)\left(a_{1}-a_{2}+a_{1} a_{2}+a_{2}^{2} \beta\right)+\left(a_{1}+a_{2} \beta\right) \sqrt{(\beta+1)\left(a_{1}+a_{2} \beta\right)}\right]}{(\beta+1)\left(a_{1}-\beta+a_{2} \beta-1\right)} \geq 0,
$$

and then we need

$$
(\beta+1)\left(a_{1}-a_{2}+a_{1} a_{2}+a_{2}^{2} \beta\right)+\left(a_{1}+a_{2} \beta\right) \sqrt{(\beta+1)\left(a_{1}+a_{2} \beta\right)} \leq 0 .
$$

Recalling (41), we must have

$$
\left\{\begin{array}{l}
\left(a_{1}-a_{2}+a_{1} a_{2}+a_{2}^{2} \beta\right) \leq 0, \\
a_{1}>\frac{(\beta+2)^{2}}{(\beta+1)}-\beta a_{2}, \\
a_{1}<4(\beta+1)-\beta a_{2}, \\
\left(a_{1}+a_{2} \beta\right)^{3}-(\beta+1)\left(a_{1}-a_{2}+a_{1} a_{2}+a_{2}^{2} \beta\right)^{2} \leq 0 .
\end{array}\right.
$$

The first inequality can be rewritten as $a_{1}\left(1+a_{2}\right) \leq a_{2}\left(1-a_{2} \beta\right)$. If $a_{2}>-1$, then we would need $a_{1} \leq$ $a_{2}\left(1-a_{2} \beta\right) /\left(1+a_{2}\right)$, which however is not possible, since $a_{2}\left(1-a_{2} \beta\right) /\left(1+a_{2}\right)<1$ and $a_{1}$, as previously noticed, must be larger then 4 .

The last inequality can be rewritten as $\left(a_{1}-\beta+a_{2} \beta-1\right)\left(a_{1}^{2}-a_{1} a_{2}^{2} \beta-a_{1} a_{2}^{2}-2 a_{1} a_{2}-a_{2}^{3} \beta^{2}-a_{2}^{3} \beta+a_{2}^{2}\right) \leq 0$. Using the second inequality, we have $a_{1}-\beta+a_{2} \beta-1>\frac{(\beta+2)^{2}}{(\beta+1)}-\beta-1=(2 \beta+3) /(\beta+1)>0$, and hence we need $a_{1}^{2}-a_{1}\left(a_{2}^{2} \beta-a_{2}^{2}-2 a_{2}\right)-a_{2}^{2}\left(a_{2} \beta^{2}-a_{2} \beta+1\right) \leq 0$. If its discriminant is negative, then the inequality is never fulfilled, so we need $a_{2}\left(a_{2}+4\right) \geq 0$. Since we can not have $a_{2}>-1$, this reduces to $a_{2}<-4$. We have

$$
a_{1}^{2}-a_{1}\left(a_{2}^{2} \beta-a_{2}^{2}-2 a_{2}\right)-a_{2}^{2}\left(a_{2} \beta^{2}-a_{2} \beta+1\right)=\left(a_{1}-r_{1}\right)\left(a_{1}-r_{2}\right),
$$


where

$$
\begin{aligned}
& r_{1}=a_{2}+\frac{a_{2}(\beta+1) \sqrt{a_{2}\left(a_{2}+4\right)}}{2}+\frac{a_{2}^{2} \beta}{2}+\frac{a_{2}^{2}}{2}, \\
& r_{2}=a_{2}-\frac{a_{2}(\beta+1) \sqrt{a_{2}\left(a_{2}+4\right)}}{2}+\frac{a_{2}^{2} \beta}{2}+\frac{a_{2}^{2}}{2} .
\end{aligned}
$$

When $a_{2}<-4$, we have $r_{2}>4(\beta+1)-\beta a_{2}$. In fact, such inequality can be equivalently rewritten as

$$
2 a_{2}-a_{2} \sqrt{a_{2}\left(a_{2}+4\right)}+a_{2}^{2}>8,
$$

which is true since the second term is positive and $a_{2}\left(2+a_{2}\right)>8$ for $a_{2}<-4$. System (42) can then be rewritten as

$$
\left\{\begin{array}{l}
a_{1} \leq a_{2} \frac{\left(1-a_{2} \beta\right)}{1+a_{2}} \\
a_{1}>\frac{(\beta+2)^{2}}{(\beta+1)}-\beta a_{2} \\
a_{1}<4(\beta+1)-\beta a_{2} \\
a_{1} \geq a_{2}+\frac{a_{2}(\beta+1) \sqrt{a_{2}\left(a_{2}+4\right)}}{2}+\frac{a_{2}^{2} \beta}{2}+\frac{a_{2}^{2}}{2} \\
a_{2}<-4 \\
a_{1}>4
\end{array}\right.
$$

A direct check shows that for $a_{2}<-4$ the first condition is less restrictive that the second and so it can be neglected. Finally, it is easy to see that for $a_{2} \in\left(-(\beta+2)^{2} /(\beta+1),-4\right)$ the fourth condition is more restrictive then the second one, while for $a_{2} \leq-(\beta+2)^{2} /(\beta+1)$ the second condition is more restrictive then the fourth one. Noticing that

$$
a_{2}+\frac{a_{2}(\beta+1) \sqrt{a_{2}\left(a_{2}+4\right)}}{2}+\frac{a_{2}^{2} \beta}{2}+\frac{a_{2}^{2}}{2}=-a_{2} \frac{1+2 \beta-\sqrt{\frac{a_{2}+4}{a_{2}}}}{\sqrt{\frac{a_{2}+4}{a_{2}}}-1},
$$

allows concluding.

In the next Proposition, which can be proved simply by suitably matching cases of the previous Lemma, we actually provide the analytical description of Figure 9

Proposition 15. We have that

- $N E=\left\{(0,0),(1,1),\left(x_{I S}^{N E}, x_{I S}^{N E}\right)\right\}$ if and only if

$$
\left\{\begin{array}{l}
\Lambda<4, \\
\Delta>\frac{4(\beta+1)}{\beta}-\frac{\Lambda}{\beta},
\end{array}\right.
$$

which defines the yellow region of Figure 9 ;

- $N E=\{(1,1)\}$ if and only if

$$
\left\{\begin{array}{l}
\Lambda>4 \\
\Delta \geq \frac{4(\beta+1)}{\beta}-\frac{\Lambda}{\beta},
\end{array}\right.
$$

which defines the blue region of Figure 9:

- $N E=\{(0,0),(1,1)\}$ if and only if

$$
\left\{\begin{array} { l } 
{ \Lambda < 4 , } \\
{ \Delta = \frac { 4 ( \beta + 1 ) } { \beta } - \frac { \Lambda } { \beta } , }
\end{array} \cup \left\{\begin{array}{l}
\Lambda=4, \\
\Delta>\frac{4(\beta+1)}{\beta}-\frac{\Lambda}{\beta},
\end{array}\right.\right.
$$

which defines the orange boundary of Figure 9 ; 
- $N E=\{(0,0)\}$ if and only if

$$
\left\{\begin{array}{l}
\Lambda \leq 4, \\
\Delta<\frac{4(\beta+1)}{\beta}-\frac{\Lambda}{\beta},
\end{array}\right.
$$

which defines the cyan region of Figure 9:

- $N E=\left\{\left(x_{I S}^{N E}, x_{I S}^{N E}\right)\right\}$ if and only if

$$
\left\{\begin{array}{l}
4<\Lambda<8 \beta+4, \\
-4 \leq-\Delta<\frac{4(\beta+1)}{\beta}-\frac{\Lambda}{\beta},
\end{array}\right.
$$

which defines the green region of Figure 9:

- $N E=\left\{\left(x_{I S}^{N E}, x_{I S}^{N E}\right),\left(x_{1, A S}^{N E}, x_{2, A S}^{N E}\right),\left(x_{2, A S}^{N E}, x_{1, A S}^{N E}\right\}\right.$ if and only if

$$
\left\{\begin{array}{l}
-\Delta \frac{\sqrt{\frac{\Delta+4}{\Delta}}+1}{1-\sqrt{\frac{\Delta+4}{\Delta}}}<\Lambda<-\Delta \frac{1+2 \beta-\sqrt{\frac{\Delta+4}{\Delta}}}{\sqrt{\frac{\Delta+4}{\Delta}}+1} \\
\Delta<-4 \\
\Lambda>4
\end{array}\right.
$$

which defines the gray region of Figure 9:

- $N E=\left\{\left(x_{I S}^{N E}, x_{I S}^{N E}\right),(\hat{x}, 0),(0, \hat{x})\right\}$ if and only if

$$
\left\{\begin{array}{l}
\Delta \leq-\Lambda \frac{1+\sqrt{\Lambda}}{\Lambda-1}, \\
4<\Lambda<(\beta+2)^{2},
\end{array}\right.
$$

which defines the dark blue region of Figure 9;

- $N E=\left\{\left(x_{I S}^{N E}, x_{I S}^{N E}\right),(0,1),(1,0)\right\}$ if and only if

$$
\left\{\begin{array}{l}
\Delta \leq \frac{(\beta+2)^{2}}{\beta(\beta+1)}-\frac{\Lambda}{\beta} \\
\Lambda \geq(\beta+2)^{2}
\end{array}\right.
$$

which defines the yellow region of Figure 9:

- $N E=\left\{\left(x_{I S}^{N E}, x_{I S}^{N E}\right),(\hat{x}, 1),(1, \hat{x})\right\}$ if and only if

$$
\left\{\begin{array} { l } 
{ - \Delta \frac { 1 + 2 \beta - \sqrt { \frac { \Delta + 4 } { \Delta } } } { 1 + \sqrt { \frac { \Delta + 4 } { \Delta } } } \leq \Lambda < \frac { 4 ( \beta + 1 ) } { \beta } - \frac { \Lambda } { \beta } , } \\
{ - \frac { ( \beta + 2 ) ^ { 2 } } { \beta + 1 } < \Delta < - 4 , }
\end{array} \cup \left\{\begin{array}{l}
\frac{(\beta+2)^{2}}{\beta(\beta+1)}-\frac{\Lambda}{\beta}<\Lambda<\frac{4(\beta+1)}{\beta}-\frac{\Lambda}{\beta}, \\
\Delta \leq-\frac{(\beta+2)^{2}}{\beta+1}
\end{array}\right.\right.
$$

which defines the orange region of Figure 9 .

Proof of Corollary 4. The existence of multiple symmetric equilibria is obtained combining cases a),b) and c) of Lemma 13. From the remaining cases we obtain the occurrence of asymmetric equilibria.

Proof of Corollary 5. Firstly we notice that if $x_{I S}^{N E}$ is actually an internal equilibrium, then, from $x_{I S}^{N E}>0$, we necessarily must have either $\gamma-\delta>4$ and $\beta \theta-2 \delta-4<0$ or $\gamma-\delta<4$ and $\beta \theta-4>0$. This means that $\gamma-\delta-4>0$ (respectively $\gamma-\delta-4<0$ ) is equivalent to $\beta \theta-2 \delta-4<0$ (respectively to $\beta \theta-2 \delta-4>0$ )

We have

$$
\frac{\partial x_{I S}^{N E}}{\partial \beta}=\frac{-2(\delta+2)}{\beta^{2}(\gamma-\delta-4)}, \quad \frac{\partial x_{I S}^{N E}}{\partial \gamma}=\frac{-2 \delta+\beta \theta-4}{\beta(\gamma-\delta-4)^{2}}, \quad \frac{\partial x_{I S}^{N E}}{\partial \theta}=-\frac{1}{\gamma-\delta-4}, \quad \frac{\partial x_{I S}^{N E}}{\partial \delta}=-\frac{\beta \theta-2 \gamma+4}{\beta(\delta-\gamma+4)^{2}}
$$


so we can immediately conclude that $\partial x_{I S}^{N E} / \partial \beta>0$ and $\partial x_{I S}^{N E} / \partial \theta>0$ if and only if $\gamma-\delta-4<0$ (or equivalently $\beta \theta-2 \delta-4>0$ ). Similarly, we have $\partial x_{I S}^{N E} / \partial \gamma>0$ if and only if $\beta \theta-2 \delta-4>0$ (or equivalently $\gamma-\delta-4<0)$. Noticing that

$$
-\beta \theta+2 \gamma-4=(-\beta \theta+2 \delta+4)+2(\gamma-\delta-4),
$$

where the last two addends have the same sign, we can conclude that $\partial x_{I S}^{N E} / \partial \delta>0$ if and only if $\gamma-\delta-4>0$ (or equivalently $\beta \theta-2 \delta-4<0$ ).

Proof of Corollary [6. Firstly we study the derivatives of $x_{1, A S}^{N E}$.

We start noticing that, since $\gamma-\delta<-4$, we have

$$
\sqrt{\frac{\gamma-\delta+4}{\gamma-\delta}}<1
$$

so

$$
\frac{\partial x_{1, A S}^{N E}}{\partial \beta}=\frac{\gamma-3 \delta+(\delta+\gamma) \sqrt{\frac{\gamma-\delta+4}{\gamma-\delta}}}{2 \beta^{2}(\gamma-\delta)}>0,
$$

since the denominator is negative and

$$
\gamma-3 \delta+(\delta+\gamma) \sqrt{\frac{\gamma-\delta+4}{\gamma-\delta}}<\gamma-3 \delta+(\delta+\gamma)=2 \gamma-2 \delta<0 .
$$

We have

$$
\frac{\partial x_{1, A S}^{N E}}{\partial \gamma}=\frac{10 \delta+2 \gamma-6 \beta \theta+\left(2 \delta^{2}-2 \delta \gamma-\beta \delta \theta+\beta \gamma \theta\right)\left(\sqrt{\frac{\gamma-\delta+4}{\gamma-\delta}}-1\right)}{2 \beta(\gamma-\delta)^{3} \sqrt{\frac{\gamma-\delta+4}{\gamma-\delta}}},
$$

where $(\gamma-\delta)^{3}<0$. Setting $a_{2}=\gamma-\delta$ and $a_{1}=\beta \theta-2 \delta$, its numerator can be rephrased into

$$
f\left(a_{1}, a_{2}\right)=2 a_{2}-6 a_{1}-a_{1} a_{2}+a_{1} a_{2} \sqrt{\frac{4}{a_{2}}+1} .
$$

A simple function study of $f\left(a_{1}, a_{2}\right)$ on $A=\left\{\left(a_{1}, a_{2}\right): a_{1}>4\right.$ and $\left.a_{2}<-4\right\}$ proves that $f\left(a_{1}, a_{2}\right)<0$,so $\partial x_{1, A S}^{N E} / \partial \gamma>0$.

We have that

$$
\frac{\partial x_{1, A S}^{N E}}{\partial \theta}=\frac{\sqrt{\frac{\gamma-\delta+4}{\gamma-\delta}}-1}{2(\gamma-\delta)}
$$

is positive recalling (43) and since $\gamma-\delta<0$.

Finally, we have

$$
\begin{aligned}
\frac{\partial x_{1, A S}^{N E}}{\partial \delta} & =\frac{2 \delta+10 \gamma-6 \beta \theta+\left(-2 \gamma^{2}+2 \delta \gamma-\beta \delta \theta+\beta \gamma \theta\right)\left(\sqrt{\frac{\gamma-\delta+4}{\gamma-\delta}}-1\right)}{2 \beta(\delta-\gamma)^{3} \sqrt{\frac{\gamma-\delta+4}{\gamma-\delta}}} \\
& =\frac{6 a_{1}-10 a_{2}+a_{2}\left(2 a_{2}-a_{1}\right)\left(\sqrt{\frac{a_{2}+4}{a_{2}}}-1\right)}{2 a_{2}^{3} \beta \sqrt{\frac{a_{2}+4}{a_{2}}}} .
\end{aligned}
$$

The denominator is negative and

$$
\begin{aligned}
6 a_{1}-10 a_{2}+a_{2}\left(2 a_{2}-a_{1}\right)\left(\sqrt{\frac{a_{2}+4}{a_{2}}}-1\right) & =a_{1}+5 a_{1}-10 a_{2}+a_{2}\left(2 a_{2}-a_{1}\right)\left(\sqrt{\frac{a_{2}+4}{a_{2}}}-1\right) \\
& =a_{1}-5\left(2 a_{2}-a_{1}\right)+a_{2}\left(2 a_{2}-a_{1}\right)\left(\sqrt{\frac{a_{2}+4}{a_{2}}}-1\right) \\
& =a_{1}+\left(2 a_{2}-a_{1}\right)\left[a_{2}\left(\sqrt{\frac{a_{2}+4}{a_{2}}}-1\right)-5\right] \\
& >\left(2 a_{2}-a_{1}\right)\left[a_{2}\left(\sqrt{\frac{a_{2}+4}{a_{2}}}-1\right)-5\right]>0,
\end{aligned}
$$


since $2 a_{2}-a_{1}<0$ and a simple study of function shows that, for $a_{2}<-4$, we have $a_{2}\left(\sqrt{\frac{a_{2}+4}{a_{2}}}-1\right)-5<0$, too. This allows concluding that $\partial x_{1, A S}^{N E} / \partial \delta<0$.

Now we focus on the derivatives of $x_{2, A S}^{N E}$. We have

$$
\frac{\partial x_{2, A S}^{N E}}{\partial \beta}=\frac{\gamma-3 \delta-(\delta+\gamma) \sqrt{\frac{\gamma-\delta+4}{\gamma-\delta}}}{2 \beta^{2}(\gamma-\delta)}>0
$$

since $\gamma-\delta<0$ and

$$
\gamma-3 \delta-(\delta+\gamma) \sqrt{\frac{\gamma-\delta+4}{\gamma-\delta}}<\gamma-3 \delta=\gamma-\delta-2 \delta<0
$$

Similarly we have

$$
\frac{\partial x_{2, A S}^{N E}}{\partial \theta}=-\frac{\sqrt{\frac{\gamma-\delta+4}{\gamma-\delta}}+1}{2(\gamma-\delta)}>0
$$

We can rewrite

$$
\frac{\partial x_{2, A S}^{N E}}{\partial \gamma}=\frac{10 \delta+2 \gamma-6 \beta \theta+\left(2 \delta \gamma-2 \delta^{2}+\beta \delta \theta-\beta \gamma \theta\right)\left(\sqrt{\frac{\gamma-\delta+4}{\gamma-\delta}}+1\right)}{2 \beta(\delta-\gamma)^{3} \sqrt{\frac{\gamma-\delta+4}{\gamma-\delta}}}
$$

as

$$
\frac{\partial x_{2, A S}^{N E}}{\partial \gamma}=\frac{6 a_{1}-2 a_{2}+a_{1} a_{2}+a_{1} a_{2} \sqrt{\frac{a_{2}+4}{a_{2}}}}{2 a_{2}^{3} \beta \sqrt{\frac{a_{2}+4}{a_{2}}}} .
$$

We recall $x_{2, A S}^{N E} \in(0,1)$ provided that

$$
A:\left\{\begin{array}{l}
-a_{2} \frac{\sqrt{\frac{a_{2}+4}{a_{2}}}+1}{1-\sqrt{\frac{a_{2}+4}{a_{2}}}}<a_{1}<-a_{2} \frac{1+2 \beta-\sqrt{\frac{a_{2}+4}{a_{2}}}}{1+\sqrt{\frac{a_{2}+4}{a_{2}}}} \\
a_{2}<-4 \\
a_{1}>4
\end{array}\right.
$$

We have that

$$
\lim _{a_{2} \rightarrow-4^{-}} \frac{\partial x_{2, A S}^{N E}}{\partial \gamma}=-\infty
$$

since

$$
\lim _{a_{2} \rightarrow-4^{-}} 2 a_{2}^{3} \beta \sqrt{\frac{a_{2}+4}{a_{2}}}=0^{-},
$$

and

$$
\lim _{a_{2} \rightarrow-4^{-}} 6 a_{1}-2 a_{2}+a_{1} a_{2}+a_{1} a_{2} \sqrt{\frac{a_{2}+4}{a_{2}}}=2 a_{1}+8>0,
$$

which means that there always exists a neighborhood of $\left(a_{1},-4\right)$, included in $A$ in which $\partial x_{1, A S}^{N E} / \partial \gamma<0$. Moreover, $\frac{\partial x_{2, A S}^{N E}}{\partial \gamma}$ vanishes only if

$$
a_{1}=z\left(a_{2}\right)=\frac{2 a_{2}}{a_{2}+a_{2} \sqrt{\frac{a_{2}+4}{a_{2}}}+6} .
$$

If $z\left(a_{2}\right)$ does not intersect $A$, thanks to the continuity of $\frac{\partial x_{2, A S}^{N E}}{\partial \gamma}$, we have that $\frac{\partial x_{2, A S}^{N E}}{\partial \gamma}<0$ on $A$. A simple computation shows that $z(-4)=-4$ lies below the lower boundary of $A$ and

$$
z\left(a_{2}\right)=-a_{2} \frac{\sqrt{\frac{a_{2}+4}{a_{2}}}+1}{1-\sqrt{\frac{a_{2}+4}{a_{2}}}}
$$


has the unique (simple) solution $a_{2}=-2(\sqrt{2}+1)$. Since $\left(a_{1}, a_{2}\right) \in A$ only if

$$
-a_{2} \frac{\sqrt{\frac{a_{2}+4}{a_{2}}}+1}{1-\sqrt{\frac{a_{2}+4}{a_{2}}}}<-a_{2} \frac{1+2 \beta-\sqrt{\frac{a_{2}+4}{a_{2}}}}{1+\sqrt{\frac{a_{2}+4}{a_{2}}}}
$$

which is equivalent to

$$
a_{2}>-\frac{(\beta+2)^{2}}{\beta+1},
$$

we need $-2(\sqrt{2}+1)>-\frac{(\beta+2)^{2}}{\beta+1}$,or equivalently $\beta>\sqrt{2}$. In this case, by continuity, we have that $\frac{\partial x_{2, A S}^{N E}}{\partial \gamma}<0$ for $a_{1}>z\left(a_{2}\right)$ and $\frac{\partial x_{2, A S}^{N E}}{\partial \gamma}>0$ for $a_{1}<z\left(a_{2}\right)$.

Finally we have

$$
\frac{\partial x_{2, A S}^{N E}}{\partial \delta}=-\frac{6 a_{1}-10 a_{2}-2 a_{2}^{2}+a_{1} a_{2}+\left(a_{1} a_{2}-2 a_{2}^{2}\right) \sqrt{\frac{a_{2}+4}{a_{2}}}}{2 a_{2}^{3} \beta \sqrt{\frac{a_{2}+4}{a_{2}}}} .
$$

We proceed as in the previous case. We have that

$$
\lim _{a_{2} \rightarrow-4^{-}} \frac{\partial x_{2, A S}^{N E}}{\partial \delta}=+\infty
$$

since

$$
\lim _{a_{2} \rightarrow-4^{-}} 2 a_{2}^{3} \beta \sqrt{\frac{a_{2}+4}{a_{2}}}=0^{-}
$$

and

$$
\lim _{a_{2} \rightarrow-4^{-}}-\left(6 a_{1}-10 a_{2}-2 a_{2}^{2}+a_{1} a_{2}+\left(a_{1} a_{2}-2 a_{2}^{2}\right) \sqrt{\frac{a_{2}+4}{a_{2}}}\right)=-2 a_{1}-8<0,
$$

which means that there always exists a neighborhood of $\left(a_{1},-4\right)$, included in $A$ in which $\partial x_{1, A S}^{N E} / \partial \delta>0$. Moreover, $\frac{\partial x_{2, A S}^{N E}}{\partial \delta}$ vanishes only if

$$
a_{1}=z\left(a_{2}\right)=\frac{10 a_{2}+2 a_{2}^{2} \sqrt{\frac{a_{2}+4}{a_{2}}}+2 a_{2}^{2}}{a_{2}+a_{2} \sqrt{\frac{a_{2}+4}{a_{2}}}+6} .
$$

If $z\left(a_{2}\right)$ does not intersect $A$, thanks to the continuity of $\frac{\partial x_{2, A S}^{N E}}{\partial \delta}$ we have that $\frac{\partial x_{2, A S}^{N E}}{\partial \delta}>0$ on $A$. A simple computation shows that $z(-4)=-4$ lies below the lower boundary of $A$ and

$$
z\left(a_{2}\right)=-a_{2} \frac{\sqrt{\frac{a_{2}+4}{a_{2}}}+1}{1-\sqrt{\frac{a_{2}+4}{a_{2}}}}
$$

has the unique (simple) solution $a_{2}=-4 \sqrt{3}-2$. Since $\left(a_{1}, a_{2}\right) \in A$ only if

$$
a_{2}>-\frac{(\beta+2)^{2}}{\beta+1}
$$

we need $-4 \sqrt{3}-2>-\frac{(\beta+2)^{2}}{\beta+1}$, or equivalently $\beta>\sqrt{3}-1$. In this case, by continuity, we have that $\frac{\partial x_{2, A S}^{N E}}{\partial \delta}>0$ for $a_{1}>z\left(a_{2}\right)$ and $\frac{\partial x_{2, A S}^{N E}}{\partial \delta}<0$ for $a_{1}<z\left(a_{2}\right)$.

Proof of Corollary 7 . Concerning $x_{0, B}^{N E}$, from

$$
x_{0, B}^{N E}=\frac{\sqrt{\beta \theta-2 \delta}-2}{\beta},
$$


we immediately have $\partial x_{0, B}^{N E} / \partial \theta>0$ and $\partial x_{0, B}^{N E} / \partial \delta<0$

$$
\frac{\partial x_{0, B}^{N E}}{\partial \beta}=\frac{4 \delta-\beta \theta+4 \sqrt{\beta \theta-2 \delta}}{2 \beta^{2} \sqrt{\beta \theta-2 \delta}}>0
$$

since for $\beta \theta-2 \delta>4$ we indeed have $4 \sqrt{\beta \theta-2 \delta}>\beta \theta-2 \delta$.

With respect to $x_{1, B}^{N E}$, from

$$
x_{1, B}^{N E}=-\frac{\beta-\sqrt{-(\beta+1)(2 \delta+\beta \delta-\beta \gamma-\beta \theta)}+2}{\beta},
$$

we have

$$
\frac{\partial x_{1, B}^{N E}}{\partial \theta}=\frac{\partial x_{1, B}^{N E}}{\partial \gamma}=\frac{\beta+1}{2 \sqrt{-(\beta+1)(2 \delta+\beta \delta-\beta \gamma-\beta \theta)}}>0
$$

and

$$
\frac{\partial x_{1, B}^{N E}}{\partial \delta}=-\frac{(\beta+1)(\beta+2)}{2 \beta \sqrt{-(\beta+1)(2 \delta+\beta \delta-\beta \gamma-\beta \theta)}}<0
$$

Moreover, we have

$$
\frac{\partial x_{1, B}^{N E}}{\partial \beta}=\frac{4 \delta+3 \beta \delta-\beta \gamma-\beta \theta+4 \sqrt{\beta \gamma-3 \beta \delta-2 \delta+\beta \theta-\beta^{2} \delta+\beta^{2} \gamma+\beta^{2} \theta}}{2 \beta^{2} \sqrt{\beta \gamma-3 \beta \delta-2 \delta+\beta \theta-\beta^{2} \delta+\beta^{2} \gamma+\beta^{2} \theta}} .
$$

Since to have $x_{1, B}^{N E} \in(0,1)$ we necessarily need $\gamma-\delta<-4$ which requires $\delta>4$ and $\beta \theta<2 \delta-\beta(\gamma-\delta)+4(\beta+1)$, we can write

$$
\begin{aligned}
4 \delta+3 \beta \delta-\beta \gamma-\beta \theta & >4 \delta+3 \beta \delta-\beta \gamma-2 \delta+\beta(\gamma-\delta)-4(\beta+1) \\
& =2 \delta+2 \beta \delta-4 \beta-4 \\
& =2 \delta-4+2 \beta(\delta-2)>0,
\end{aligned}
$$

since $\gamma-\delta<-4$ guarantees $\delta>4$. This concludes the proof.

\section{Proofs of Section 3.3}

We start noticing that, from (16), we have

$$
\begin{gathered}
\partial_{x_{i}} \pi_{i}\left(x_{i}, x_{j}\right)=\frac{-\alpha \beta x_{j}^{2}+\beta x_{j}+1}{\left(\beta x_{i}+\beta x_{j}-2 \alpha \beta x_{i} x_{j}+2\right)^{2}} \theta \beta-1, \\
\partial_{x_{i}}^{2} \pi_{i}\left(x_{i}, x_{j}\right)=\frac{2 \beta^{2} \theta\left(2 \alpha x_{j}-1\right)\left(-\alpha \beta x_{j}^{2}+\beta x_{j}+1\right)}{\left(\beta x_{i}+\beta x_{j}-2 \alpha \beta x_{i} x_{j}+2\right)^{3}} \\
\partial_{x_{i} x_{j}}^{2} \pi_{i}\left(x_{i}, x_{j}\right)=\frac{\beta^{2} \theta(4 \alpha+\beta)\left(x_{i}-x_{j}\right)}{\left(\beta x_{i}+\beta x_{j}-2 \alpha \beta x_{i} x_{j}+2\right)^{3}} .
\end{gathered}
$$

As for the proof of the previous sections, we introduce a suitable function $x_{+}:[0,1 / 2 \alpha) \rightarrow \mathbb{R}$ defined by

$$
x_{+}\left(x_{j}\right)=-\frac{2}{\beta\left(1-2 \alpha x_{j}\right)}-\frac{x_{j}}{\left(1-2 \alpha x_{j}\right)}+\frac{\sqrt{\beta \theta\left(-\alpha \beta x_{j}^{2}+\beta x_{j}+1\right)}}{\beta\left(1-2 \alpha x_{j}\right)},
$$

which will be used in the definition of the best response. Function $x_{+}$is investigated in the following Lemma. Notice that for $x_{j} \in[0,1 / 2 \alpha)$ we have $-\alpha \beta x_{j}^{2}+\beta x_{j}+1>0$, so (46) is well-defined.

Lemma 14. We have that

a) $x_{+}\left(x_{j}\right) \leq 0$ for any $x_{j} \in[0,1 / 2 \alpha)$ if and only if $\beta \theta \leq 4$. 
b) If

$$
4<\beta \theta<4+\frac{\beta}{\alpha},
$$

then $x_{+}$is unimodal and concave in $[0,1 / 2 \alpha)$, and attains its maximum for

$$
z_{m}=\frac{\beta-\sqrt{\beta(4 \alpha+\beta-\alpha \beta \theta)}}{2 \alpha \beta}
$$

at which $x_{+}\left(z_{m}\right)=z_{m}$. Moreover

$$
\lim _{x_{j} \rightarrow\left(\frac{1}{2 \alpha}\right)^{-}} x_{+}\left(x_{j}\right)=-\infty
$$

c) If

$$
\beta \theta \geq 4+\frac{\beta}{\alpha},
$$

then $x_{+}$is strictly increasing in $[0,1 / 2 \alpha)$. Moreover

$$
\lim _{x_{j} \rightarrow\left(\frac{1}{2 \alpha}\right)^{-}} x_{+}\left(x_{j}\right)= \begin{cases}\frac{1}{2 \alpha} & \text { if } \beta \theta=4+\beta / \alpha \\ +\infty & \text { if } \beta \theta>4+\beta / \alpha .\end{cases}
$$

Proof. a) From

$$
x_{+}(0)=\frac{\sqrt{\beta \theta}-2}{\beta},
$$

we have that $x_{+}(0) \leq 0$ if and only if $\beta \theta \leq 4$. Moreover, for $\beta \theta \leq 4$ we have

$$
x_{+}^{\prime}\left(x_{j}\right)=\frac{(4 \alpha+\beta)}{2 \beta\left(2 \alpha x_{j}-1\right)^{2} \sqrt{\beta \theta\left(-\alpha \beta x_{j}^{2}+\beta x_{j}+1\right)}}\left(\beta \theta-2 \sqrt{\beta \theta\left(-\alpha \beta x_{j}^{2}+\beta x_{j}+1\right)}\right)<0 .
$$

In fact, the first factor is positive, while the second one is negative since, noticing that $-\alpha \beta x_{j}^{2}+\beta x_{j}<0$ for $x_{j}<1 / 2 \alpha$, we have

$$
\beta \theta-2 \sqrt{\beta \theta\left(-\alpha \beta x_{j}^{2}+\beta x_{j}+1\right)}<\beta \theta-2 \sqrt{\beta \theta},
$$

and the last term is non positive for $\beta \theta \leq 4$.

b) We have

$$
x_{+}^{\prime \prime}=\frac{(4 \alpha+\beta) \sqrt{\theta}\left(16 \alpha\left(-\alpha \beta x_{j}^{2}+\beta x_{j}+1\right) \sqrt{\beta\left(-\alpha \beta x_{j}^{2}+\beta x_{j}+1\right)}+\sqrt{\theta}\left(\beta^{2}-8 \alpha \beta+12 \alpha^{2} \beta^{2} x_{j}^{2}-12 \alpha \beta^{2} x_{j}\right)\right)}{4 \beta\left(2 \alpha x_{j}-1\right)^{3}\left(-\alpha \beta x_{j}^{2}+\beta x_{j}+1\right) \sqrt{\beta \theta\left(-\alpha \beta x_{j}^{2}+\beta x_{j}+1\right)}} .
$$

We notice that the denominator is negative. If $\left(\beta^{2}-8 \alpha \beta+12 \alpha^{2} \beta^{2} x_{j}^{2}-12 \alpha \beta^{2} x_{j}\right) \geq 0$, then $x_{+}^{\prime \prime}\left(x_{j}\right)<0$, conversely, if $\left(\beta^{2}-8 \alpha \beta+12 \alpha^{2} \beta^{2} x_{j}^{2}-12 \alpha \beta^{2} x_{j}\right)<0$, we have that the numerator is positive provided that

$$
\theta<\frac{256 \alpha^{2}\left(-\alpha \beta x_{j}^{2}+\beta x_{j}+1\right)^{3}}{\beta\left(-12 \beta \alpha^{2} x_{j}^{2}+12 \beta \alpha x_{j}+8 \alpha-\beta\right)^{2}}
$$

From (47), we have $\theta \leq(4 \alpha+\beta) / \alpha \beta$. Moreover,

$$
\frac{4 \alpha+\beta}{\alpha \beta}<\frac{256 \alpha^{2}\left(-\alpha \beta x_{j}^{2}+\beta x_{j}+1\right)^{3}}{\beta\left(-12 \beta \alpha^{2} x_{j}^{2}+12 \beta \alpha x_{j}+8 \alpha-\beta\right)^{2}}
$$

can be equivalently rewritten as

$$
\frac{\beta\left(2 \alpha x_{j}-1\right)^{4}\left(16 \beta \alpha^{2} x_{j}^{2}-16 \beta \alpha x_{j}-12 \alpha+\beta\right)}{\alpha\left(-12 \beta \alpha^{2} x_{j}^{2}+12 \beta \alpha x_{j}+8 \alpha-\beta\right)^{2}}<0,
$$


and since $16 \beta \alpha^{2} x_{j}^{2}-16 \beta \alpha x_{j}-12 \alpha+\beta<\beta-8 \alpha+12 \alpha^{2} \beta x_{j}^{2}-12 \alpha \beta x_{j}$ is equivalent to $4 \alpha\left(\beta \alpha x_{j}^{2}-\beta x_{j}-1\right)<0$ which is true for $x_{j} \in\left[0,1 / 2 \alpha\right.$ ), we have that (52) is fulfilled, and then (51) is satisfied. This proves that $x_{+}$ is unimodal and concave.

Imposing $x_{+}\left(x_{j}\right)=x_{j}$, after some computations we obtain

$$
4 \alpha^{2} \beta^{2} x_{j}^{4}-8 \alpha \beta^{2} x_{j}^{3}+\beta(4 \beta-8 \alpha+\theta \alpha \beta) x_{j}^{2}+\beta(8-\theta \beta) x_{j}+4-\beta \theta=0,
$$

which can be rewritten as

$$
4 \alpha^{2} \beta^{2}\left(x_{j}^{2}-\frac{1}{\alpha} x_{j}-\frac{1}{\alpha \beta}\right)\left(x_{j}^{2}-\frac{1}{\alpha} x_{j}-\frac{1}{\alpha \beta}+\frac{\theta}{4 \alpha}\right)=0,
$$

whose solutions are

$$
\frac{1}{2 \alpha}+\frac{\sqrt{\beta(4 \alpha+\beta)}}{2 \alpha \beta}, \frac{\beta-\sqrt{\beta(4 \alpha+\beta)}}{2 \alpha \beta}, \frac{1}{2 \alpha}+\frac{\sqrt{\beta(4 \alpha+\beta-\alpha \beta \theta)}}{2 \alpha \beta}, \frac{1}{2 \alpha}-\frac{\sqrt{\beta(4 \alpha+\beta-\alpha \beta \theta)}}{2 \alpha \beta} .
$$

Notice that, thanks to the assumption $(4 \alpha+\beta-\alpha \beta \theta)>0$, they are all real. However, the first and the third solution are larger than $1 / 2 \alpha$ and the second one is negative. Conversely, the last one is indeed smaller than $1 / 2 \alpha$ and, since we assumed $\beta \theta \geq 4$, it is also non negative.

Finally, recalling (47), a simple computation of $\lim _{x_{j} \rightarrow\left(\frac{1}{2 \alpha}\right)^{-}} x_{+}\left(x_{j}\right)$ allows concluding.

c) Using the expression of $x_{+}^{\prime}$ computed in (50), it is easy to see that $x_{+}^{\prime}\left(x_{j}\right)>0$ can be equivalently rewritten as

$$
4 \alpha \beta x_{j}^{2}-4 \beta x_{j}+\beta \theta-4>0 .
$$

Noticing that the left hand side of the previous inequality is strictly decreasing for $x_{j} \in[0,1 / 2 \alpha)$ and that its limit for $x \rightarrow(1 / 2 \alpha)^{-}$is $\beta \theta-\frac{\beta}{\alpha}-4 \geq 0$ (thanks to (49)), we have that $x_{+}^{\prime}\left(x_{j}\right)>0$. Finally, recalling (49) a simple computation of $\lim _{x_{j} \rightarrow\left(\frac{1}{2 \alpha}\right)^{-}} x_{+}\left(x_{j}\right)$ allows concluding.

As we shall see, for games $\Gamma \in \Gamma^{L S E}$ the best response relation is not always a function. To suitably study it, we divide the investigation of $B R_{i}$ into two parts. In the next Lemma we consider $\alpha>1 / 2$, for which $B R_{i}$ is a function for any $x_{j} \in[0,1]$, and $\alpha \leq 1 / 2$, for which $B R_{i}\left(x_{j}\right)$ is a function only for $x_{j} \in[0,1 / 2 \alpha)$.

Lemma 15. Let either $\alpha>1 / 2$ and $x_{j} \in[0,1]$ or $\alpha \leq 1 / 2$ and $x_{j} \in[0,1 / 2 \alpha)$. Then we have $B R_{i}\left(x_{j}\right)=$ $\max \left\{\min \left\{x_{+}\left(x_{j}\right), 1\right\}, 0\right\}$.

Proof. If $\alpha>1 / 2$ and $x_{j} \in[0,1]$ or $\alpha \leq 1 / 2$ and $x_{j} \in[0,1 / 2 \alpha)$, from 45 we have $\partial_{x_{i}}^{2} \pi_{i}\left(x_{i}, x_{j}\right)<0$ since $2 \alpha x_{j}-1<0,-\alpha \beta x_{j}^{2}+\beta x_{j}+1<0$ and $\beta x_{i}+\beta x_{j}-2 \alpha \beta x_{i} x_{j}+2>0$, which means that $\pi_{i}$ is concave with respect to $x_{i} \in[0,1]$. The only possible solutions of $\partial_{x_{i}} \pi_{i}\left(x_{i}, x_{j}\right)=0$ are

$$
x_{ \pm}\left(x_{j}\right)=\frac{-2-\beta x_{j} \pm \sqrt{\beta \theta\left(-\alpha \beta x_{j}^{2}+\beta x_{j}+1\right)}}{\beta\left(1-2 \alpha x_{j}\right)},
$$

where $x_{-}$is strictly negative. Proceeding as for the proofs of Lemmas 2 and 6 , we can conclude that $B R_{i}\left(x_{j}\right)=\max \left\{\min \left\{x_{+}\left(x_{j}\right), 1\right\}, 0\right\}$.

In the next Lemma we study $B R_{i}$ for $\alpha \geq 1 / 2$ and $x_{j} \in[1 / 2 \alpha, 1]$ (notice that for $\alpha=1 / 2$ such interval reduces to $\{1\})$.

Lemma 16. Let $\alpha \geq 1 / 2$ and $x_{j} \in[1 / 2 \alpha, 1]$. We have that

a) if $\beta \theta<4+\beta / \alpha$, then $B R_{i}\left(x_{j}\right)=0$ 
b) if $\beta \theta=4+\beta / \alpha$ then $B R_{i}\left(x_{j}\right)= \begin{cases}{[0,1]} & x_{j}=\frac{1}{2 \alpha} \\ 0 & \frac{1}{2 \alpha}<x_{j}=1\end{cases}$

c) if $4+\beta / \alpha<\beta \theta \leq 2 \beta+4$ then $B R_{i}\left(x_{j}\right)= \begin{cases}1 & \frac{1}{2 \alpha} \leq x_{j}<\tilde{x}_{j} \\ \{0,1\} & x_{j}=\tilde{x}_{j} \\ 0 & \tilde{x}_{j}<x_{j} \leq 1\end{cases}$

d) if $\beta \theta>2 \beta+4$ then $B R_{i}\left(x_{j}\right)=1$

Proof. We divide the proof into two cases.

Firstly, we study $x_{j}=1 / 2 \alpha$. In this case, we can take $\alpha \geq 1 / 2$ and we have

$$
\pi_{i}\left(x_{i}, \frac{1}{2 \alpha}\right)=\frac{-x_{i}(4 \alpha+\beta-\alpha \beta \theta)-2 \alpha \theta-\beta \theta}{4 \alpha+\beta} .
$$

This means that if $4 \alpha+\beta-\alpha \beta \theta>0$, then $B R_{i}(1 / 2 \alpha)=0$, if $4 \alpha+\beta-\alpha \beta \theta<0$ then $B R_{i}(1 / 2 \alpha)=1$ and if $4 \alpha+\beta-\alpha \beta \theta=0$ then $B R_{i}(1 / 2 \alpha)=[0,1]$. In particular

a) if $4 \alpha+\beta-\alpha \beta \theta>0$, then (54) is strictly decreasing and $B R_{i}(1 / 2 \alpha)=0$;

b) if $4 \alpha+\beta-\alpha \beta \theta=0$, then (54) is constant and $B R_{i}(1 / 2 \alpha)=[0,1]$;

$\mathrm{c}, \mathrm{d})$ if $4 \alpha+\beta-\alpha \beta \theta<0$, then (54) is strictly increasing and $B R_{i}(1 / 2 \alpha)=1$.

Now we consider $x_{j} \in(1 / 2 \alpha, 1]$. In this case, to have a non-empty interval, we must take $\alpha>1 / 2$. We start studying the sign of $f\left(x_{j}\right)=\pi_{i}\left(0, x_{j}\right)-\pi_{i}\left(1, x_{j}\right)$, given by

$$
f\left(x_{j}\right)=\beta^{2}(1-2 \alpha+\alpha \theta) x_{j}^{2}+\beta(4-4 \alpha+\beta-\beta \theta) x_{j}+4+2 \beta-\beta \theta .
$$

A simple computation shows that

$$
\begin{gathered}
f\left(\frac{1}{2 \alpha}\right)=\frac{(4 \alpha+\beta)(4 \alpha+\beta-\alpha \beta \theta)}{4 \alpha^{2}}, \\
f(1)=(2 \beta-\beta \theta+4)(\beta-\alpha \beta+1), \\
f^{\prime}\left(x_{j}\right)=2 \beta^{2}(1-2 \alpha+\alpha \theta) x_{j}-\beta(4 \alpha-\beta+\beta \theta-4) .
\end{gathered}
$$

a) If $\beta \theta<4+\beta / \alpha$, then both $f(1 / 2 \alpha)>0$ and $f(1)>0$. Moreover, we have that if $(1-2 \alpha+\alpha \theta)=0$, then $f\left(x_{j}\right)=\left((4 \alpha+\beta)\left(\beta x_{2}(1-\alpha)+1\right)\right) / \alpha>0$, and then $B R_{i}\left(x_{j}\right)=0$ for any $x_{j} \in(1 / 2 \alpha, 1]$.

Conversely, if $(1-2 \alpha+\alpha \theta)<0$, since $f$ is concave and both $f(1 / 2 \alpha)>0$ and $f(1)>0$, then $B R_{i}\left(x_{j}\right)=0$ for any $x_{j} \in(1 / 2 \alpha, 1]$.

Finally, if $(1-2 \alpha+\alpha \theta)>0, f$ is convex but $f^{\prime}(1 / 2 \alpha)=\beta(4 \alpha+\beta)(1-\alpha) / \alpha>0$, which, together with $f(1 / 2 \alpha)>0$, guarantees that $B R_{i}\left(x_{j}\right)=0$ for any $x_{j} \in(1 / 2 \alpha, 1]$.

b) If $\beta \theta=4+\beta / \alpha$, we have $(1-2 \alpha+\alpha \theta)=2-2 \alpha+4 \alpha / \beta>0$, so $f$ is strictly convex and, as just seen, increasing. Since $f(1 / 2 \alpha)=0$ and $f(1)>0$, we have that $f\left(x_{j}\right)>0$ (i.e. $B R_{i}\left(x_{j}\right)=0$ ) for each $x_{j} \in(1 / 2 \alpha, 1]$.

c) If $4+\beta / \alpha<\beta \theta<2 \beta+4$, we have $f(1 / 2 \alpha)<0, f(1)>0$ and $f^{\prime}\left(x_{j}\right)>0$. By continuity and increasing monotonicity of $f$, we have exactly one solution of $f\left(x_{j}\right)=0$ belonging to $(1 / 2 \alpha, 1)$, given by (17). Recalling that for $\beta \theta>4+\beta / \alpha, x_{+}$is strictly convex, we have

- $x_{j}<\tilde{x}_{j}$ we have $f\left(x_{j}\right)=\pi_{i}\left(0, x_{j}\right)-\pi_{i}\left(1, x_{j}\right)<0$ and then $B R_{i}\left(x_{j}\right)=1$;

- $x_{j}=\tilde{x}_{j}$ we have $f\left(x_{j}\right)=\pi_{i}\left(0, x_{j}\right)-\pi_{i}\left(1, x_{j}\right)=0$ and then $B R_{i}\left(x_{j}\right)=\{0,1\}$;

- $x_{j}>\tilde{x}_{j}$ we have $f\left(x_{j}\right)=\pi_{i}\left(0, x_{j}\right)-\pi_{i}\left(1, x_{j}\right)>0$ and then $B R_{i}\left(x_{j}\right)=0$. 
Noticing that for $\beta \theta=2 \beta+4$ we have both $f(1)=0$, which means that $B R_{i}(1)=\{0,1\}$, and $\tilde{x}_{j}=1$, we can conclude.

d) If $\beta \theta>2 \beta+4$, we have $f(1 / 2 \alpha)<0, f(1)<0$ and $f^{\prime}\left(x_{j}\right)>0$, which means that $f\left(x_{j}\right)<0$ (i.e. $\left.B R_{i}\left(x_{j}\right)=1\right)$ for each $x_{j} \in(1 / 2 \alpha, 1]$.

Lemma 17. Let $\alpha>1 / 2$. If $B R_{i}\left(\tilde{x}_{j}\right)=0$ for some $\tilde{x}_{j} \in[0,1]$, then $B R_{i}\left(x_{j}\right)=0$ for any $x_{j} \in\left[\tilde{x}_{j}, 1\right]$.

If $B R_{i}\left(\tilde{x}_{j}\right)=1$ for some $\tilde{x}_{j} \in[0,1]$, then $B R_{i}\left(x_{j}\right)=1$ for any $x_{j} \in\left[\tilde{x}_{j}, 1\right]$.

Proof of Proposition [5. Combining Lemmas 15 and 16 we immediately obtain the four possible expressions and characterizations (18), (19), (20) and (21) of the best response relation. In particular, we stress that upper-hemicontinuity for the cases in which $B R_{i}$ is a correspondence is a consequence of the closed-graph theorem, since it is easy to see that in such cases correspondence $B R_{i}$ is closed and indeed has both compact domain and range.

If $\alpha>1 / 2$, the same arguments used for the proof of Proposition 1 are still valid and provide either strictly non-increasing, hump-shaped or constantly equal to 0,1 best response functions.

If $\alpha \geq 1 / 2$ and $\beta \theta<4+\frac{\beta}{\alpha}$, the best response function is hump-shaped. If $\alpha \geq 1 / 2$ and $\beta \theta=4+\frac{\beta}{\alpha}$, we have that $B R_{i} \equiv x_{+}$is strictly increasing in $[0,1 / 2 \alpha)$, while $B R_{i} \equiv 0$ on $(1 / 2 \alpha, 1]$. If $\alpha \geq 1 / 2$ and $\beta \theta>4+\frac{\beta}{\alpha}$, we have that $B R_{i}$ is non-decreasing in $[0,1 / 2 \alpha)$ with $B R_{i}(0)<1$ and $B R_{i}\left(x_{j}\right)=1$ for $x_{j} \in\left[1 / 2 \alpha, \tilde{x}_{j}\right)$. Then $B R_{i}\left(x_{j}\right)=0$ for $x_{j} \in\left(\tilde{x}_{j}, 1\right]$.

The analytical characterization of the best response function shape with respect to the parameters, which is graphically reported in Figure 5 is investigated in the next Proposition.

Proposition 16. Best response functions of games $\Gamma \in \Gamma^{L S E}$ are

a) constantly equal to 0 if and only if $\beta \theta \leq 4$, which defines the blue region in Figure 10 .

b) hump-shaped if and only if

$$
\left\{\begin{array} { l } 
{ \alpha < \frac { 1 } { 2 } } \\
{ 4 < \beta \theta < 4 ( \beta + 1 - \alpha \beta ) }
\end{array} \cup \left\{\begin{array}{l}
\alpha \geq \frac{1}{2} \\
4<\beta \theta<4+\frac{\beta}{\alpha}
\end{array}\right.\right.
$$

which defines the white region in Figure 10

c) strictly non-decreasing and then null if and only if

$$
\left\{\begin{array}{l}
\alpha \geq \frac{1}{2} \\
4+\frac{\beta}{\alpha} \leq \beta \theta \leq 2 \beta+4
\end{array}\right.
$$

which defines the pink region in Figure 10.

d) strictly non-decreasing if and only if

$$
\left\{\begin{array} { l } 
{ \alpha < \frac { 1 } { 2 } } \\
{ 4 ( \beta + 1 - \alpha \beta ) \leq \beta \theta < ( \beta + 2 ) ^ { 2 } }
\end{array} \cup \left\{\begin{array}{l}
\alpha \geq \frac{1}{2} \\
2 \beta+4<\beta \theta<(\beta+2)^{2}
\end{array}\right.\right.
$$

which defines the green region in Figure 10.

e) $B R_{i} \equiv 1$ if and only if $\beta \theta \geq(\beta+2)^{2}$, which defines the cyan region in Figure 10 . 
Proof. a) If $\beta \theta \leq 4$,from Lemmas [14, 15] and [16, we have that $x_{+}\left(x_{j}\right) \leq 0$ for $x_{j} \in[0,1 / 2 \alpha) \cap[0,1]$ and $B R_{i}\left(x_{j}\right)=0$ for $x_{j} \in[1 / 2 \alpha, 1]$.

b) If $\alpha<1 / 2$ and $4<\beta \theta<4(\beta+1-\alpha \beta)<4+\beta / \alpha$ we have that, from Lemmas 14 and 15, $B R_{i}\left(x_{j}\right)=$ $x_{+}\left(x_{j}\right)$ for any $x_{j} \in[0,1]$. Moreover, it is easy to see, from (48), that $0<z_{m}<1$ is equivalent to $4<\beta \theta<4(\beta+1-\alpha \beta)$. If $\alpha \geq 1 / 2$, from the previous considerations, applying Lemmas 14, 15 and 16 and noticing that in this case $4(\beta+1-\alpha \beta) \geq 4+\beta / \alpha$, we can conclude.

c) Since $\beta \theta \geq 4+\frac{\beta}{\alpha}$, we have that $x_{+}$is strictly increasing in $[0,1 / 2 \alpha)$. Since $\beta \theta>4$, we have $x_{+}(0)>0$. If $\beta \theta=4+\beta / \alpha$ we have that $\lim _{x_{j} \rightarrow(1 / 2 \alpha)^{-}} x_{+}\left(x_{j}\right)<1$, and then, since $B R_{i}\left(x_{j}\right)=x_{+}\left(x_{j}\right)$ we have the the best response function is strictly non-increasing for $x_{j} \in[0,1 / 2 \alpha)$ and, from Lemma 16, $B R_{i}\left(x_{j}\right)=0$ for $x_{j} \in(1 / 2 \alpha, 1]$. Conversely, if $\beta \theta>4+\beta / \alpha$, since $\lim _{x_{j} \rightarrow(1 / 2 \alpha)^{-}} x_{+}\left(x_{j}\right)=+\infty$, we have that there exists $b \in(0,1 / 2 \alpha)$ such that $B R_{i}\left(x_{j}\right)=x_{+}\left(x_{j}\right)$ for $x_{j} \in[0, b], B R_{i}\left(x_{j}\right)=1$ for $x_{j} \in[b, 1 / 2 \alpha)$. From Lemma 16 we also have that $B R_{i}\left(x_{j}\right)=1$ for $x_{j} \in\left[1 / 2 \alpha, \tilde{x}_{j}\right)$, which guarantees a strictly non-decreasing best response function for $x_{j} \in\left[0, \tilde{x}_{j}\right)$. Finally, again from Lemma 16 we have $B R_{i}\left(x_{j}\right)=0$ for $x_{j} \in\left(\tilde{x}_{j}, 1\right]$.

d) For $4(\beta+1-\alpha \beta) \leq \beta \theta<(\beta+2)^{2}$, it is easy to see that $x_{+}(0) \in(0,1)$. For $\alpha<1 / 2$, since $z_{m}>1$, $x_{+}$is increasing in $[0,1]$. This means that there exists $b \in(0,1)$ such that $B R_{i}\left(x_{j}\right)=x_{+}\left(x_{j}\right)$ for $x_{j} \in[0, b]$, $B R_{i}\left(x_{j}\right)=1$ for $x_{j} \in[b, 1]$. If $\alpha \geq 1 / 2$, since $2 \beta+4>4+\beta / \alpha$, from Lemma 14, $x_{+}$is increasing in $[0,1 / 2 \alpha)$. Since $\lim _{x_{j} \rightarrow(1 / 2 \alpha)^{-}} x_{+}\left(x_{j}\right)=+\infty$, we have that there exists $b \in(0,1 / 2 \alpha)$ such that $B R_{i}\left(x_{j}\right)=x_{+}\left(x_{j}\right)$ for $x_{j} \in[0, b], B R_{i}\left(x_{j}\right)=1$ for $x_{j} \in[b, 1 / 2 \alpha)$. A simple computation shows that for $\beta \theta>2 \beta+4$ we have $\tilde{x}_{j}>1$, which from Lemma [16] means that $B R_{i}\left(x_{j}\right)=1$ for $x_{j} \in[1 / 2 \alpha, 1]$.

e) For $\beta \theta \geq(\beta+2)^{2}$ we have $x_{+}(0) \geq 1$. Since, from Lemma 14, we have that, for both $\alpha<1 / 2$ and $\alpha \geq 1 / 2, x_{+}\left(x_{j}\right)$ is strictly increasing for $x_{j} \in[0,1 / 2 \alpha)$, we have $B R_{i}\left(x_{j}\right)=1$ for $x_{j} \in[0,1 / 2 \alpha) \cap[0,1]$. If $\alpha \geq 1 / 2$, from Lemma 16] since a simple computation show that $\tilde{x}_{j}>1$, we have that $B R_{i}\left(x_{j}\right)=1$ for $x_{j} \in[1 / 2 \alpha, 1]$.

Since the previous inequalities provide a partition of the parameters' space, we have that all the previous sufficient conditions are also necessary.

Now we prove Proposition 6 .

Proof of Proposition [6. The cases of hump-shaped, strictly non-decreasing and constant best response functions can be handled proceeding exactly as in the proof of Proposition 2 since the best response functions of both games $\Gamma^{L N S}$ and $\Gamma^{L S E}$ share the same characteristics. In the last remaining case the best response is a correspondence whose expression is given by either (20) or (21). In the former case, using $\beta \theta=4+\beta / \alpha$, it is easy to see that $B R_{i}\left(x_{j}\right)>x_{j}$ for $x_{j} \in[0,1 / 2 \alpha)$, while it is and null for $x_{j} \in(1 / 2 \alpha, 1]$. So we do not have any equilibria for $x_{j} \neq 1 / 2 \alpha$. Since $B R_{i}(1 / 2 \alpha)=[0,1]$, the unique Nash equilibrium is $(1 / 2 \alpha, 1 / 2 \alpha)$, which is internal for $\alpha<1$ and becomes the corner equilibrium $(1,1)$ for $\alpha=1$. Noticing that $x_{I S}^{N E}=1 / 2 \alpha$ for $\beta \theta=4+\beta / \alpha$ allows concluding the first part of the proof. Conversely, if $\beta \theta>4+\beta / \alpha$, we have $B R_{i}\left(x_{j}\right)>x_{j}$ for $x_{j} \in[0,1 / 2 \alpha), B R_{i}\left(x_{j}\right)=1$ for $x_{j} \in\left[1 / 2 \alpha, \tilde{x}_{j}\right)$ and $B R_{i}\left(x_{j}\right)=0$ for $x_{j} \in\left(\tilde{x}_{j}, 1\right]$. So we can not have equilibria for $x_{j} \neq \tilde{x}_{j}$. For $x_{j}=\tilde{x}_{j}$ we have $B R_{i}\left(\tilde{x}_{j}\right)=\{0,1\}$, which means that we may have an equilibrium if and only if either $\tilde{x}_{j}=0$ (which is not possible since $\tilde{x}_{j}>1 / 2 \alpha$ ) or $\tilde{x}_{j}=1$, Imposing $\tilde{x}_{j}=1$, we find $\theta=(2 \beta+4) / \beta$, which is compatible with $\beta \theta>4+\beta / \alpha$ for $\alpha>1 / 2$. Then, when $\alpha>1 / 2$ and $\theta=(2 \beta+4) / \beta$ and $\beta \theta>4+\beta / \alpha$, we have the unique corner equilibrium $(1,1)$.

Using Proposition [16 and the proof of Proposition [6 we obtain the analytical description of regions depicted in Figure 12 which is reported in the following Proposition.

Proposition 17. We have that

a) $(0,0)$ is the unique equilibrium if and only if $\beta \theta \leq 4$, which defines the cyan region in Figure 12. 
b) $\left(x_{I S}^{N E}, x_{I S}^{N E}\right)$ is the unique equilibrium if and only if

$$
\left\{\begin{array} { l } 
{ \alpha \leq \frac { 1 } { 2 } } \\
{ 4 < \beta \theta < 4 ( \beta + 1 - \alpha \beta ) }
\end{array} \cup \left\{\begin{array}{l}
\alpha>\frac{1}{2} \\
4<\beta \theta \leq 4+\frac{\beta}{\alpha}
\end{array}\right.\right.
$$

which defines the green region in Figure 12.

c) there is no equilibrium if and only if

$$
\left\{\begin{array}{l}
\alpha>\frac{1}{2} \\
4+\frac{\beta}{\alpha}<\beta \theta<2 \beta+4
\end{array}\right.
$$

which defines the pink region in Figure 12,

d) $(1,1)$ is the unique equilibrium if and only if

$$
\left\{\begin{array} { l } 
{ \alpha < \frac { 1 } { 2 } } \\
{ \beta \theta \geq 4 ( \beta + 1 - \alpha \beta ) }
\end{array} \cup \left\{\begin{array}{l}
\alpha \geq \frac{1}{2} \\
\beta \theta \geq 2 \beta+4
\end{array}\right.\right.
$$

which defines the blue region in Figure [12.

Proof. Since in Proposition 17 we showed that the equilibrium is unique, if we prove the sufficiency of the previous conditions, which provide a partition of the $(\beta, \alpha, \theta)$ space, we automatically have that they are necessary too.

a) It is sufficient to notice that from $\beta \theta \leq 4$ we have that $B R_{i}(0)=0$ (Lemma 14).

b) We start considering the couple of inequalities given by case c) of Proposition 16. Under such assumption, we have hump-shaped best response functions, which, recalling Proposition [6 provide the unique internal symmetric equilibrium.

We only have to consider $\alpha>\frac{1}{2}$ and $\beta \theta=4+\frac{\beta}{\alpha}$. In the proof of Proposition [6 we showed that in this case $(1 / 2 \alpha, 1 / 2 \alpha)$ is the internal symmetric equilibrium. This concludes the proof of case $b)$.

c) The result is obtained in the proof of Proposition 6

d) The result is a consequence of cases d) and e) of Proposition 16 and of the proof of Proposition 6 , 